\title{
HYPOELLIPTICITY ON THE HEISENBERG GROUP-REPRESENTATION-THEORETIC CRITERIA
}

\author{
BY \\ CHARLES ROCKLAND ${ }^{1}$
}

\begin{abstract}
A representation-theoretic characterization is given for hypoellipticity of homogeneous (with respect to dilations) left-invariant differential operators $P$ on the Heisenberg group $H_{n}$; it is the precise analogue for $H_{n}$ of the statement for $\mathbf{R}^{n}$ that a homogeneous constant-coefficient differential operator is hypoelliptic if and only if it is elliptic. Under these representation-theoretic conditions a parametrix is constructed for $\boldsymbol{P}$ by means of the Plancherel formula. However, these conditions involve all the irreducible representations of $H_{n}$, whereas only the generic, infinite-dimensional representations occur in the Plancherel formula. A simple class of examples is discussed, namely $P=\sum_{i=1}^{n} X_{i}^{2 m}+Y_{i}^{2 m}$, where $X_{i}, Y_{i}, i=1, \ldots, n$, and $Z$ generate the Lie algebra of $H_{n}$ via the commutation relations $\left[X_{i}, Y_{j}\right]=\delta_{i j} Z$, and where $m$ is a positive integer. In the course of the proof a connection is made between homogeneous left-invariant operators on $H_{n}$ and a class of degenerate-elliptic operators on $\mathbf{R}^{n+1}$ studied by Grusin. This connection is examined in the context of localization in enveloping algebras.
\end{abstract}

1. Introduction. The main purpose of this paper is to present a representation-theoretic characterization of hypoellipticity for homogeneous (with respect to dilations) left-invariant differential operators $P$ on the Heisenberg group $H_{n}$.

Definition 1.1. A differential operator $P$ on a manifold $M$ is hypoelliptic if and only if for any distribution $u \in \mathscr{D}^{\prime}(M)$, s.s. $u \subseteq$ s.s. $P u$, where s.s. denotes singular support. That is, if $P u$ is $C^{\infty}$ on the open set $\Omega \subseteq M$, then $u$ is $C^{\infty}$ on $\Omega$.

The principal result is the following:

THEOREM 1.2. Let $P$ be a left-invariant homogeneous differential operator on the Heisenberg group $H_{n}$. Then the following are equivalent:

(1.1) $P$ and $P^{t}$, the formal transpose of $P$, are both hypoelliptic.

Received by the editors September 10, 1976.

AMS (MOS) subject classifications (1970). Primary 22E25, 22E45, 35D05, 35H05, 43A80; Secondary $16 \mathrm{C} 25$.

Key words and phrases. Heisenberg group, nilpotent Lie group, unitary representations, Plancherel formula, dilations, homogeneous left-invariant differential operator, hypoellipticity, parametrix.

'Research partially supported by NSF grant MPS-71-02759A05. 
(1.2) For every irreducible unitary representation $\pi$ of $H_{n}$ (except the 1dimensional identity representation), $\pi(P)$ has a bounded two-sided inverse. Here $\pi(P)$ is viewed as an unbounded linear operator in the Hilbert-space $H$ of $\pi$, with domain the space of $C^{\infty}$-vectors of the representation $\pi$.

(1.3) For every irreducible unitary representation $\pi$ of $H_{n}$ (except the 1dimensional identity representation), $\pi(P) v \neq 0$ and $\pi(P)^{*} v \neq 0$, for every nonzero $C^{\infty}$-vector $v$ of the representation $\pi$.

Theorem 1.2 is simply the analogue for $G=H_{n}$ of the following wellknown result for $G=\mathbf{R}^{n}$, the translation group, endowed with the usual family of dilations: A homogeneous constant-coefficient differential operator is hypoelliptic if and only if it is elliptic. (For details see \$5.) The idea of focusing attention on homogeneous differential operators is due to Stein [23], and also, in a different context, to Grušin [9].

Our work relies heavily on the representation theory, in particular, on the Plancherel formula, for $H_{n}$, which we examine in the general context of the Kirillov orbit picture. A basic ingredient in the proof of Theorem 1.2 is the use of the Plancherel formula to construct a parametrix for $P^{t}$, the formal transpose of $P$, which is $C^{\infty}$ away from the identity element. However, only the "generic" representations of $H_{n}$ occur in the Plancherel formula, and if one tries to use the formula to write down a fundamental solution for $P^{t}$, a serious convergence problem arises as the generic representations approach the hyperplane of "degenerate" representations, i.e., as Planck's constant approaches 0 . It is the hypothesis that $\pi(P)$ is invertible for "degenerate" $\pi$ which allows us to circumvent this difficulty. Roughly speaking, with this hypothesis we are able to find $W$ in the center of the enveloping algebra such that $P^{t}+W$ is elliptic, and to construct $u \in \mathcal{D}^{\prime}(G)$, with the Plancherel formula, satisfying $P^{t} u=\left(P^{t}+W\right) \delta$. Convolving $u$ with a compactly supported parametrix for $P^{t}+W$, we obtain a parametrix for $P^{t}$. In earlier work relating hypoellipticity with representation theory [7], [21], the invertibility hypothesis for the degenerate representations is satisfied implicitly, and, consequently, the significance of the "degenerate" representations has been overlooked. (See [11], however.)

As the reader will see, the methods we use are rather "formal", in the sense that they are not crucially tied to $H_{n}$, but should, with appropriate modification, go over to more general simply-connected nilpotent Lie groups $G$ with "dilations". (Precisely how general is not yet clear. See \$8.) Certainly the Kirillov orbit picture, in particular the use of the center of the enveloping algebra to parametrize the orbits, is valid in full generality, and the dilations can be naturally incorporated into this scheme. We consider the present paper a first step towards the proof of the general case, and, consequently, shall keep this general context in view throughout the exposition. 
We proceed to an outline of the paper.

In $\$ 2$ we sketch some basic results concerning representation theory for simply-connected nilpotent Lie groups, including the Plancherel theorem.

In $\$ 3$ we work specifically on $H_{n}$. We show, under the hypothesis that $\pi(P)$ has a bounded left-inverse for every $\pi$ except the 1-dimensional identity representation, how to construct a parametrix for $P^{t}$.

In $\$ 4$, assuming some results from $\S 6$, we shall show that, under the stronger hypothesis (1.2), the parametrix for $P^{t}$ constructed in $\S 3$ is $C^{\infty}$ away from the identity, and hence that $P$ is hypoelliptic. The same argument, but with $P$ and $P^{t}$ interchanged, shows that $P^{t}$ is hypoelliptic. Actually, it seems very likely that the left-invertibility of $\pi(P)$ should be sufficient to guarantee hypoellipticity of $P$. We return to this point in $\$ 6$. We should observe also that if we know that $P$ and $P^{t}$ are both hypoelliptic, and if we appropriately restrict the degree of homogeneity, then, by work of Folland [6], we can deduce much additional information.

In $\$ 5$ we prove that if $P$ and $P^{t}$ are both hypoelliptic then (1.3) holds. This, together with the fact, to be proved in $\$ 6$, that (1.2) and (1.3) are equivalent, completes the proof of Theorem 1.2.

There is a very close and natural relationship between operators studied by Grušin in [9] and homogeneous left-invariant operators on nilpotent Lie groups. Indeed, there is a very direct link between Theorem 1.2 and the results of Grušin [9]; in fact it was the discovery of the group-theoretic interpretation of the results of Grušin which led us to conjecture Theorem 1.2, and, in particular, directed our attention to the "degenerate" representations.

In $\$ 6$ we study this relationship. We feel it is of interest in its own right, but we include it here primarily because some of the consequences are needed in $\S \S 4$ and 5.

There is an interesting class of operators $P$ for which it is easy to verify that (1.3) holds. We examine this class in $\$ 7$, where we derive a strengthened form of the following proposition:

Proposition 1.3. Let $G$ be a simply-connected nilpotent Lie group, with Lie algebra $g$. Let $X_{1}, \ldots, X_{k}$ be elements of $g$ whose repeated commutators span $g$. Then for any positive integers $m_{i}, i=1, \ldots, k$, all even or all odd, the operator $P=\sum_{i=1}^{k} X_{i}^{2 m_{i}}$ satisfies (1.3) (with $H_{n}$ replaced by $G$ ).

These operators, with $m_{i} \equiv 1$, have received a good deal of attention, both in the group-theoretic context and in the more general "variable-coefficient" context. (See, for example, [13], [6], [22].)

At least in the case of $G=H_{n}$ we can, by Theorem 1.2, say that such $P$, if homogeneous, are hypoelliptic. We thus have

Corollary 1.4. Let $X_{1}, \ldots, X_{n}, Y_{1}, \ldots, Y_{n}, Z$ be the standard generators 
for the Lie algebra of $H_{n}$, i.e., $\left[X_{i}, Y_{j}\right]=\delta_{i j} Z$, and $\left[X_{i}, Z\right]=0,\left[Y_{i}, Z\right]=0$. Then for any positive integer $m$, the operator $P=\sum_{i=1}^{n}\left(X_{i}^{2 m}+Y_{i}^{2 m}\right)$ is hypoelliptic.

We conclude in $\$ 8$ with a discussion of open problems.

2. Preliminaries on nilpotent Lie groups. We assume that the reader has some familiarity with basic representation theory, and shall review only those points which will be needed later. As general references we shall use [2] and [15].

Let $G$ be a simply-connected nilpotent Lie group, with Lie algebra $g$ and (complexified) universal enveloping algebra $U(g)$. Since $G$ is simply-connected the exponential map exp: $g \rightarrow G$ is a diffeomorphism. In fact [2] $G$ is unimodular, i.e., left Haar measure equals right Haar measure, and is the image under exp of Lebesgue measure on $g$.

We identify $g$ with the left-invariant real vector fields on $G$ by associating to $X \in g$ the vector-field, still denoted $X$, defined by

$$
(X \varphi)(x)=\left.\frac{d}{d t}\right|_{t=0} \varphi(x \exp t X), \quad \varphi \in C^{\infty}(G) .
$$

This identification extends uniquely to an isomorphism between the algebra $\mathcal{U}(g)$ and the algebra of all left-invariant differential operators on $G$ (with complex coefficients) (see [12]). Thus, every left-invariant differential operator on $G$ is of the form

$$
P=\sum_{|\alpha|<m} a_{\alpha} X_{1}^{\alpha_{1}} X_{2}^{\alpha_{2}} \cdots X_{N}^{\alpha_{N}}, \quad a_{\alpha} \in \mathrm{C}, X_{i} \in g .
$$

If $X_{1}, \ldots, X_{n}$ form an ordered basis for $g$ then the expansion (2.2) is unique by the Birkhoff-Poincaré-Witt theorem.

If $P$ is a differential operator on $G$, then by $P^{t}$ we mean the formal transpose of $P$ with respect to Haar measure, and by $P^{*}$ the formal adjoint. Thus, $P^{*}=(\bar{P})^{t}=\left(\overline{\left.P^{t}\right)}\right.$, where $\bar{P}$ is defined by $\bar{P}(\varphi)=\overline{P(\bar{\varphi})}$, - denoting complex-conjugation.

Since translation is an isometry with respect to Haar measure it follows that if $X \in g$ then

$$
X^{t}=-X .
$$

Thus, if $P \in \mathcal{U}(\mathrm{g})$ is given by (2.2),

$$
\begin{gathered}
\bar{P}=\sum_{|\alpha|<m} \bar{a}_{\alpha} X_{1}^{\alpha_{1}} X_{2}^{\alpha_{2}} \cdots X_{N}^{\alpha_{N}}, \\
P^{t}=\sum_{|\alpha|<m} a_{\alpha}\left(-X_{N}\right)^{\alpha_{N}}\left(-X_{N-1}\right)^{\alpha_{N-1}} \cdots\left(-X_{1}\right)^{\alpha_{1}},
\end{gathered}
$$




$$
P^{*}=\sum_{|\alpha|<m} \bar{a}_{\alpha}\left(-X_{N}\right)^{\alpha_{N}}\left(-X_{N-1}\right)^{\alpha_{N-1}} \cdots\left(-X_{1}\right)^{\alpha_{1}} .
$$

Of course, $\bar{P}, P^{t}, P^{*}$ also lie in $थ(g)$.

If $\pi$ is a unitary representation of $G$ on the Hilbert space $H$, then $v \in H$ is called a $C^{\infty}$-vector for $\pi$ if the map $x \mapsto \pi(x) v$ from $G$ to $H$ is $C^{\infty}$. The $C^{\infty}$-vectors form a vector subspace of $H$, which we denote by $H_{\infty}$. The representation $\pi$ determines a Lie algebra representation $\pi$ of $g$ as linear maps: $H_{\infty} \rightarrow H_{\infty}$ defined by

$$
\pi(X) v=\left.\frac{d}{d t}\right|_{t=0} \pi(\exp t X) v, \quad X \in g, v \in H_{\infty} .
$$

This extends uniquely to a representation of the algebra $U(g)$ as linear maps: $H_{\infty} \rightarrow H_{\infty}$. (If $c \in \mathrm{C}, \pi(c)=c$.) If $\pi$ is irreducible then [15] there is a unitarequivalence taking $H$ to $L^{2}\left(\mathbf{R}^{n}\right)$ for some $n$ (possibly 0 ) and taking $H_{\infty}$ to $\delta\left(\mathbf{R}^{n}\right)$, the Schwartz space.

The summable functions $L^{1}(G)$ form an algebra under convolution, defined by

$$
(f * g)(x)=\int_{G} f\left(x y^{-1}\right) g(y) d y=\int_{G} f(y) g\left(y^{-1} x\right) d y
$$

where $d y$ denotes Haar measure. If $\pi$ is a unitary representation of $G$ on $H$, then $\pi$ determines a representation of the algebra $L^{1}(G)$ as bounded linear operators on $H$ by

$$
\pi(f) v=\int_{G} f(y) \pi(y) v d y, \quad v \in H .
$$

To say that $\pi$ is an algebra representation means, in particular,

$$
\pi(f * g)=\pi(f) \pi(g) \text {. }
$$

It is clear from (2.8) that, letting $\|\cdot\|$ denote bounded operator norm,

$$
\|\pi(f)\| \leqslant\|f\|_{L^{\prime}(G)}
$$

Kirillov [15] shows that if $\pi$ is irreducible, $\pi(f)$ is a compact operator for $f \in L^{1}(G)$, and if $\varphi \in C_{0}^{\infty}(G)$, then $\pi(\varphi)$ is of trace-class. Also, $\pi(\varphi)$ maps $H$ into $H_{\infty}$, even if $\pi$ is not irreducible. The space spanned by $\{\pi(\varphi) v \mid \varphi \in$ $C_{0}^{\infty}(G), v \in H$, called the Gårding subspace, is dense in $H$, and hence, so is $H_{\infty}$.

Lemma 2.1. Let $\varphi \in C_{0}^{\infty}(G)$ and $X \in g$. Then for any unitary representation $\pi$ of $G$ and any $C^{\infty}$-vector $v$,

$$
\pi(X \varphi) v=\pi(\varphi) \pi(-X) v .
$$


Proof.

$$
\begin{aligned}
\pi(X \varphi) v & =\int_{G}(X \varphi)(x) \pi(x) v d x=\left.\int_{G} \frac{d}{d t}\right|_{t=0} \varphi(x \exp t X) \pi(x) v d x \\
& =\left.\frac{d}{d t}\right|_{t=0} \int_{G} \varphi(x \exp t X) \pi(x) v d x \\
& =\left.\frac{d}{d t}\right|_{t=0} \int_{G} \varphi(x) \pi(x \exp -t X) v d x \quad \text { (by right-translation) } \\
& =\left.\frac{d}{d t}\right|_{t=0} \int_{G} \varphi(x) \pi(x) \pi(\exp -t X) v d x \\
& =\int_{G} \varphi(x) \pi(x)\left(\left.\frac{d}{d t}\right|_{t=0} \pi(\exp -t X) v\right) d x \\
& =\int_{G} \varphi(x) \pi(x) \pi(-X) v d x=\pi(\varphi) \pi(-X) v .
\end{aligned}
$$

LEMMA 2.2. For any unitary representation $\pi$ of $G$, and for any $X \in g$, $\pi(-X)=\pi(X)^{*}$ (where * denotes formal Hilbert-space adjoint). That is, $\langle\pi(X) v, w\rangle=\langle v, \pi(-X) w\rangle$ for every $v, w \in H_{\infty}$.

Proof. Since $\pi$ is a unitary representation of $G, \pi$ is skew-adjoint on $g$. Thus, $\pi(X)^{*}=-\pi(X)=\pi(-X)$.

Since $X \in g$ is real it follows from (2.3) that $X^{*}=-X$. Hence Lemma 2.2 states that $\pi\left(X^{*}\right)=\pi(X)^{*}$. A simple argument using (2.6) now shows

(2.12) For any $P \in \mathcal{U}(g), \pi\left(P^{*}\right)=\pi(P)^{*}$.

Similarly, an argument based on Lemma 2.1 and (2.5) shows

(2.13) For any $P \in \mathcal{Q}(g)$, for any $\varphi \in C_{0}^{\infty}(G)$, and for any $C^{\infty}$-vector $v$, $\pi(P \varphi) v=\pi(\varphi) \pi\left(P^{t}\right) v$.

It follows immediately that

$$
\begin{aligned}
\pi\left(P^{t} \varphi\right) v & =\pi(\varphi) \pi(P) v \\
\pi\left(P^{*} \varphi\right) v & =\pi(\varphi) \pi(\bar{P}) v \\
\pi\left(\bar{P}_{\varphi}\right) v & =\pi(\varphi) \pi(P)^{*} v
\end{aligned}
$$

For $\varphi \in C^{\infty}(G)$ we define $\check{\varphi}, \varphi^{\#} \in C^{\infty}(G)$ by

$$
\check{\varphi}(x)=\varphi\left(x^{-1}\right) ; \quad \varphi^{\#}(x)=\overline{\varphi\left(x^{-1}\right)} .
$$

LEMMA 2.3. For any $\varphi \in C_{0}^{\infty}(G)$ and any unitary representation $\pi$ of $G$, $\pi\left(\varphi^{\#}\right)=\pi(\varphi)^{*}$. 
ProOF.

$$
\begin{aligned}
\pi\left(\varphi^{\#}\right) & =\int_{G} \overline{\varphi\left(x^{-1}\right)} \pi(x) d x \\
& =\int_{G} \overline{\varphi(x)} \pi\left(x^{-1}\right) d x \quad \text { (since Haar measure is unimodular) } \\
& =\int_{G} \overline{\varphi(x)} \pi(x)^{*} d x=\left[\int_{G} \varphi(x) \pi(x) d x\right]^{*}=\pi(\varphi)^{*} . \quad \square
\end{aligned}
$$

Combining this with (2.9) we see

(2.18) For any $\varphi \in C_{0}^{\infty}(G), \pi\left(\varphi *\left(\varphi^{\#}\right)\right)=\pi(\varphi) \pi(\varphi)^{*}$.

We also derive the following.

(2.19) For any $\varphi \in C_{0}^{\infty}(G), \varphi * \varphi^{\#}(e)=\|\varphi\|_{L^{2}(G)}^{2}=\left\|\varphi^{\#}\right\|_{L^{2}(G)}^{2}$, where $e$ denotes the identity element of $G$.

Proof. Using the second definition of convolution we get

$$
\varphi * \varphi^{\#}(e)=\int_{G} \varphi(y) \varphi^{\#}\left(y^{-1}\right) d y=\int_{G} \varphi(y) \overline{\varphi(y)} d y=\|\varphi\|_{L^{2}(G)}^{2}
$$

Since $G$ is unimodular,

$$
\begin{aligned}
\int_{G} \varphi(y) \overline{\varphi(y)} d y & =\int_{G} \varphi\left(y^{-1}\right) \overline{\varphi\left(y^{-1}\right)} d y \\
& =\int_{G} \overline{\varphi^{\#}(y)} \varphi^{\#}(y) d y=\left\|\varphi^{\#}\right\|_{L^{2}(G)}^{2}
\end{aligned}
$$

We shall find it necessary when we deal with hypoellipticity questions to be able to apply unitary representations of $G$ to compactly-supported distributions on $G$, denoted $\mathcal{E}^{\prime}(G)$. To do so it is convenient to introduce a variant of the notion of $C^{\infty}$-vector.

DEFINITION 2.4. Let $\pi$ be a unitary representation of $G$ on $H$. We say that $v \in H$ is a weak $C^{\infty}$-vector for $\pi$ if it satisfies the following two conditions: $C^{\infty}$.

(2.20) For every $w \in H$ the map $\varphi_{v, w}: G \rightarrow \mathbf{C}$ given by $x \mapsto\langle\pi(x) v, w\rangle$ is

(2.21) The map $w \mapsto \varphi_{v, w}$ from $H$ to $C^{\infty}(G)$ is continuous with respect to the norm topology on $H$ and the usual topology on $C^{\infty}(G)$ (uniform convergence on compact subsets of all partial derivatives).

REMARKS. 1. Clearly the weak $C^{\infty}$-vectors for $\pi$ form a linear subspace, $H_{\infty}^{w}$, of $H$.

2. Every $C^{\infty}$-vector is certainly weak $C^{\infty}$ as well, i.e., $H_{\infty} \subseteq H_{\infty}^{w}$. In fact, at least when $\pi$ is irreducible, the two spaces coincide. (See Note 6 following Proposition 2.6.)

DefintIION 2.5. Let $\pi$ be a unitary representation of $G$ on $H$, and let $u \in \mathcal{E}^{\prime}(G)$. We define $\pi(u)$ as a possibly unbounded linear operator on $H$ with domain $H_{\infty}^{w}$ as follows. For any $v \in H_{\infty}^{w}, \pi(u) v$ is the unique vector in $H$ 
satisfying

$$
\langle\pi(u) v, w\rangle=\left(u, \varphi_{v, w}\right) \text { for every } w \in H .
$$

We check that this definition makes sense. Since $\varphi_{v, w}$ is in $C^{\infty}(G),\left(u, \varphi_{v, w}\right)$ is a well-defined complex number. For fixed $u \in \mathcal{E}^{\prime}(G)$ and $v \in H_{\infty}^{w}$ the function $w \mapsto\left(u, \varphi_{v, w}\right)$ from $H$ to $C$ is clearly conjugate-linear and, because of (2.21), continuous. Therefore for fixed $u, v$ there is a unique vector $\pi(u) v \in H$ such that (2.22) holds. Clearly the map $v \mapsto \pi(u) v$ is linear.

REMARK. It is probably possible to extend $\pi(u)$ naturally to a larger domain than $H_{\infty}^{w}$. For example, although I have not carefully checked this, it is probably possible to extend $\pi(u)$ to a linear map from $H$ to the dual space $H_{\infty}^{\prime}$ of $H_{\infty}$ with the "Schwartz-space topology." (See Kirillov [15, p. 62]). Thus by the remark following (2.7), if $\pi$ were irreducible $\pi(u)$ would give a linear map: $L^{2}\left(\mathbf{R}^{n}\right) \rightarrow \mathcal{S}^{\prime}\left(\mathbf{R}^{n}\right)$, the space of tempered distributions. Also, by putting the Schwartz-space topology on $H_{\infty}$ we can probably make $\pi(u)$ : $H_{\infty} \rightarrow H_{\infty}^{\prime}$ continuous. Again, I have not checked this carefully since we shall not need this information in the case when $G$ is the Heisenberg group.

Many of the earlier results for $\pi(\varphi), \varphi \in C_{0}^{\infty}(G)$, continue to hold for $\pi(u)$. We bring various of these together in the next proposition.

First, define $\check{u}, u^{\#} \in \mathcal{E}^{\prime}(G)$ for $u \in \mathcal{E}^{\prime}(G)$ by

$$
(\check{u}, \psi)=(u, \check{\psi}), \quad\left(u^{\#}, \psi\right)=\overline{\left(u, \psi^{\#}\right)} \quad \text { for every } \psi \in C^{\infty}(G) .
$$

A simple verification, using the fact that Haar measure is unimodular, shows that this definition agrees with (2.17) for $u \in C_{0}^{\infty}(G)$.

Proposition 2.6.(1) If $f \in L^{1}(G) \cap \mathcal{E}^{\prime}(G)$, then $\pi(f)$, where $f$ is viewed as an element of $\mathcal{E}^{\prime}(G)$ is the "same" operator as in the original definition of $\pi(f)$; more precisely, $\pi(f)$, defined on the dense subspace $H_{\infty}^{w}$ of $H$, extends uniquely to a bounded operator on $H$ which equals the original $\pi(f)$.

(2) For any $u \in \mathcal{E}^{\prime}(G), \pi\left(u^{\#}\right)=\pi(u)^{*}$. More precisely, for any $v, w \in H_{\infty}^{w}$, $\langle\pi(u) v, w\rangle=\left\langle v, \pi\left(u^{\#}\right) w\right\rangle$.

(3) For any $u \in \mathcal{E}^{\prime}(G), \pi(u)$ maps $H_{\infty}^{w}$ into itself.

(4) If $u \in \mathcal{E}^{\prime}(G)$ and $\varphi \in C_{0}^{\infty}(G)$, then $\pi(u * \varphi)=\pi(u) \pi(\varphi)$, both sides being viewed as bounded operators on $H$.

(5) If $u_{1}, u_{2}$ are in $\mathcal{E}^{\prime}(G)$, then $\pi\left(u_{1} * u_{2}\right)=\pi\left(u_{1}\right) \pi\left(u_{2}\right)$, both sides viewed as (unbounded) operators defined on $H_{\infty}^{w}$.

(6) For any $P \in \mathcal{U}(g)$ and any $u \in \mathcal{E}^{\prime}(G), \pi(P u)=\pi(u) \pi\left(P^{t}\right)$, viewed as (unbounded) operators on $H_{\infty}$.

(7) For any unitary representation $\pi, \pi(\delta)=I$, the identity operator on $H$. Here $\delta$ is the $\delta$-function supported at the identity element.

Notes. 1. We assume the reader is familiar with the notion of convolution of distributions which appears in(4) and (5). In particular, in (4) $u * \varphi \in$ 
$C_{0}^{\infty}(G)$ since $\varphi \in C_{0}^{\infty}(G)$, and so $\pi(u * \varphi)$ is a bounded operator on $H$. By the remarks following (2.10), $\pi(\varphi)$ maps $H$ into $H_{\infty}$, and similarly for $\pi(u * \varphi)$. In particular, $\pi(u) \pi(\varphi)$ is defined on all of $H$. Note that (4) shows that $\pi(u) \pi(\varphi) v$ is in $H_{\infty}$, in fact in the Gårding subspace, for any $v \in H$. That is, $\pi(u)$ maps the Gårding subspace into itself.

2. The right-hand side of (5) is meaningful because of (3). I do not know whether (3) remains true, in general, with $H_{\infty}^{w}$ replaced by $H_{\infty}$. This is the reason for introducing the space $H_{\infty}^{w}$. However, see Note 6 below.

3. In (6) we work with $H_{\infty}$, since this is the usual domain for $\pi(P)$. It is easy to see, however, that the domain for $\pi(P)$ can be taken as $H_{\infty}^{w}$, and that (6) is valid on this domain. For example, we could use (2.24) below to define $\pi(P) v$ for $v \in H_{\infty}^{w}$.

4. Of course (7) means that $\pi(\delta)$ extends to $I$.

5. Using (7) we obtain a useful special case of (6):

(2.24) For every $P \in \mathcal{U}(g), \pi(P)=\pi\left(P^{\prime} \delta\right)$.

6. If $\pi$ is irreducible then $H_{\infty}^{w}=H_{\infty}$. In particular, by (3), for any $u \in \mathcal{E}^{\prime}(G), \pi(u)$ maps $H_{\infty}$ into itself. Clearly, it suffices to prove this for any unitarily equivalent representation. Thus, since $\pi$ is irreducible we may assume without loss of generality (see [15]) that $H=L^{2}\left(\mathbf{R}^{n}\right)$ for some $n$, that $H_{\infty}=\mathcal{S}\left(\mathbf{R}^{n}\right)$, the Schwartz space, and that $\{\pi(P) \mid P \in \mathcal{U}(g)\}=A_{n}(\mathbf{C})$, the algebra of all differential operators on $\mathbf{R}^{n}$ with polynomial coefficients. From (2) and (2.24) we see that for every $P \in \mathcal{Q}(g), v \in H_{\infty}^{w}$ and $w \in H_{\infty}$, $\langle\pi(P) v, w\rangle=\left\langle v, \pi\left(\left(P^{t} \delta\right)^{\#}\right) w\right\rangle$. Taking both $v$ and $w$ in the above to be in $H_{\infty}$, we see from (2.12) that $\pi\left(\left(P^{t} \delta\right)^{\#}\right) w=\pi\left(P^{*}\right) w$. Hence $\langle\pi(P) v, w\rangle=$ $\left\langle v, \pi\left(P^{*}\right) w\right\rangle$ for any $v \in H_{\infty}^{w}, w \in H_{\infty}$. This equation says that $\pi(P) v \in$ $L^{2}\left(\mathbf{R}^{n}\right)$, defined via (2.22), coincides with the tempered distribution in $\mathcal{S}^{\prime}\left(\mathbf{R}^{n}\right)$ obtained by applying the differential operator with polynomial coefficients, $\pi(P)$, to $v \in L^{2}\left(\mathbf{R}^{n}\right)$ viewed as an element of $\mathcal{S}^{\prime}\left(\mathbf{R}^{n}\right)$. Thus, in particular, $Q v \in L^{2}\left(\mathbf{R}^{n}\right)$ for every differential operator $Q \in A_{n}(\mathbf{C})$. It follows from the Sobolev lemma that $Q v \in C^{k}\left(\mathbf{R}^{n}\right)$ for every $Q \in A_{n}(\mathbf{C})$ and every positive integer $k$, where $C^{k}\left(\mathbf{R}^{n}\right)$ is space of all $k$-times continuously-differentiable functions on $\mathbf{R}^{n}$ whose derivatives up through order $k$ are bounded. This clearly implies that $v \in \mathcal{S}\left(\mathbf{R}^{n}\right)=H_{\infty}$.

Proof of Proposition. (1) Obvious.

(2) Straightforward generalization of proof of Lemma 2.3.

(3) We need to show that for any $v \in H_{\infty}^{w}$ the following hold:

(2.25) For any $w \in H$, the map $\varphi_{\pi(u) v, w}: G \rightarrow \mathrm{C}$ is $C^{\infty}$. But

(2.26) The map $w \mapsto \varphi_{\pi(u) v, w}$ from $H$ to $C^{\infty}(G)$ is continuous.

$$
\varphi_{\pi(u) v, w}(x)=\langle\pi(x) \pi(u) v, w\rangle=\left\langle\pi(u) v, \pi(x)^{*} w\right\rangle=\left(u, \varphi_{v, \pi(x)^{*} w}\right),
$$

and 


$$
\varphi_{v, \pi(x)^{*} w}(y)=\left\langle\pi(y) v, \pi(x)^{*} w\right\rangle=\langle\pi(x y) v, w\rangle=\varphi_{v, w}(x y) .
$$

That is, $\varphi_{\pi(u) v, w}(x)=\left(u_{y}, \varphi_{0, w}(x y)\right)$, i.e. $u$ applied to the $C^{\infty}$-function of $y$, $\varphi_{v, w}(x y)$. Since this equals $u * \check{\varphi}_{v, w}\left(x^{-1}\right)$, it is $C^{\infty}$. This proves (2.25). But for any $u \in \mathcal{E}^{\prime}(G)$ the map $\varphi \mapsto u * \varphi$ from $C^{\infty}(G)$ to $C^{\infty}(G)$ is continuous; also the map $\check{v}: C^{\infty}(G) \rightarrow C^{\infty}(G)$ is continuous; Hence, since we know that the map $w \stackrel{\mapsto}{\rightarrow} \varphi_{v, w}$ from $H$ to $C^{\infty}(G)$ is continuous, and since $\varphi_{\pi(u) v, w}=$ $\left(u * \check{\varphi}_{v, w}\right)^{\sim},(2.26)$ follows.

(4) We want to show that

$$
\langle\pi(u * \varphi) v, w\rangle=\langle\pi(u) \pi(\varphi) v, w\rangle \text { for all } v, w \in H .
$$

For $v, w \in H, \varphi_{v, w}(x) \equiv\langle\pi(x) v, w\rangle$, although not a $C^{\infty}$ function on $G$, is certainly continuous (as part of the definition of unitary representation) and, in particular, a distribution. Thus, since

$$
\begin{gathered}
\langle\pi(u * \varphi) v, w\rangle=\int_{G}(u * \varphi)(x) \varphi_{v, w}(x) d x \\
\langle\pi(u * \varphi) v, w\rangle=(u * \varphi) * \check{\varphi}_{v, w}(e)=u *\left(\varphi * \check{\varphi}_{v, w}\right)(e)=\left(u,\left(\varphi * \check{\varphi}_{v, w}\right)^{\nu}\right) .
\end{gathered}
$$

On the other hand, by definition of $\pi(u),\langle\pi(u) \pi(\varphi) v, w\rangle=\left(u, \varphi_{\pi(\varphi) v, w}\right)$. Thus, to prove (2.27) it suffices to show that for any $v, w \in H, \varphi_{\pi(\varphi) v, w}=$ $\left(\varphi * \check{\varphi}_{v, w}\right)$, a relation we derived in a more general form in the proof of (3), with $\varphi$ replaced by $u$, but with $v$ restricted to $H_{\infty}^{w}$. A straightforward computation shows that the desired relation holds for arbitrary $v \in H$.

(5) Now let $u_{1}, u_{2} \in \mathcal{E}^{\prime}(G)$, and $\varphi \in C_{0}^{\infty}(G)$. Then $\left(u_{1} * u_{2}\right) * \varphi=$ $u_{1} *\left(u_{2} * \varphi\right)$, and since $u_{2} * \varphi \in C_{0}^{\infty}(G)$, it follows from (4) that

$$
\pi\left(u_{1} * u_{2}\right) \pi(\varphi)=\pi\left(u_{1}\right) \pi\left(u_{2} * \varphi\right)=\pi\left(u_{1}\right) \pi\left(u_{2}\right) \pi(\varphi)
$$

In particular, for any $v \in H$ and $w \in H_{\infty}^{w}$,

$$
\left\langle\pi\left(u_{1} * u_{2}\right) \pi(\varphi) v, w\right\rangle=\left\langle\pi\left(u_{1}\right) \pi\left(u_{2}\right) \pi(\varphi) v, w\right\rangle .
$$

Therefore, by (2) and (3),

$$
\left\langle\pi(\varphi) v, \pi\left(\left(u_{1} * u_{2}\right)^{\#}\right) w\right\rangle=\left\langle\pi(\varphi) v, \pi\left(u_{2}^{\#}\right) \pi\left(u_{1}^{\#}\right) w\right\rangle .
$$

But an easy argument shows that $\left(u_{1} * u_{2}\right)^{\#}=u_{2}^{\#} * u_{1}^{\#}$. So, for any $v \in H$ and any $w \in H_{\infty}^{w}$,

$$
\left\langle\pi(\varphi) v, \pi\left(u_{2}^{\#} * u_{1}^{\#}\right) w\right\rangle=\left\langle\pi(\varphi) v, \pi\left(u_{2}^{\#}\right) \pi\left(u_{1}^{\#}\right) w\right\rangle .
$$

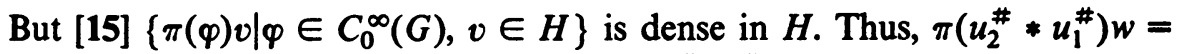
$\pi\left(u_{2}^{\#}\right) \pi\left(u_{1}^{\#}\right) w$ for any $w \in H_{\infty}^{w}$. Replacing $u_{1}^{\#}, u_{2}^{\#}$ by $u_{1}, u_{2}$, we prove (5).

(6) Let $u \in \mathcal{E}^{\prime}(G)$ and $P \in \mathcal{Q}(g) . P u=P(u * \delta)$. Since $P$ is left-invariant this equals $u * P \delta$. Thus, by $(5), \pi(P u)=\pi(u) \pi(P \delta)$. Let $v \in H_{\infty}$ and $w \in H$. Then

$$
\langle\pi(P \delta) v, w\rangle=\left(P \delta, \varphi_{v, w}\right)=\left(\delta, P^{t} \varphi_{v, w}\right)=\left.P^{t}(\langle\pi(x) v, w\rangle)\right|_{x=e^{*}}
$$


It follows from (2.7) that this equals $\left\langle\pi\left(P^{t}\right) v, w\right\rangle$. Thus, for any $v \in H_{\infty}$, $\pi(P \delta) v=\pi\left(P^{t}\right) v$, and so, by the above, $\pi(P u) v=\pi(u) \pi\left(P^{t}\right) v$.

(7) $\left(\delta, \varphi_{v, w}\right)=\varphi_{v, w}(e)=\langle\pi(e) v, w\rangle=\langle v, w\rangle$.

We next turn to the Plancherel theorem. We shall need to use it both in its distributional and in its $L^{2}$ formulation. The version we present below is a compilation of results in Dixmier [2] and Kirillov [15]. Dixmier presents a sharp formulation of the $L^{2}$-version, and Kirillov provides a satisfying geometrical exposition of the distributional version. The reader is referred to [2] and [15] for details and further information (also, see Kirillov [16].)

THEOREM 2.7. Let $G$ be a simply-connected nilpotent Lie group of dimension $N$. Let $g$ denote the Lie algebra, $g^{*}$ the dual of the Lie algebra, U $(g)$ the complexified enveloping algebra, and $Z(g)$ the center of the enveloping algebra. Every coadjoint orbit in $g^{*}$ is even dimensional. Let $2 n$ be the maximal dimension which occurs, and let $q=N-2 n$. Let $W_{1}, \ldots, W_{q}$ be selfadjoint, algebraically independent elements of $Z(g)$ which generate the field of fractions of $Z(g)$. Then there exists a nonempty Zariski-open subset $\Gamma$ of $\mathbf{R}^{q}$, and for every $\lambda=\left(\lambda_{1}, \ldots, \lambda_{q}\right) \in \Gamma$ a unitary irreducible representation $\pi_{\lambda}$ of $G$ in $H=L^{2}\left(\mathbf{R}^{n}\right)$ so that the following properties are satisfied.

(1) For every $\lambda \in \Gamma$ and any $i=1, \ldots, q, \pi_{\lambda}\left(W_{i}\right)=\lambda_{i} I$; moreover, any unitary irreducible representation of $G$ satisfying this property is unitarily equivalent to $\pi_{\lambda}$.

(2) For every $\lambda \in \Gamma$, the space of $C^{\infty}$-vectors for $\pi_{\lambda}$ is $\delta\left(\mathbf{R}^{n}\right)$.

(3) For every $\lambda \in \Gamma$, the algebra of operators $\pi_{\lambda}(Q)$ as $Q$ runs through $थ(g)$ is $A_{n}(\mathrm{C})$, the Weyl-algebra, consisting of all differential operators on $\mathbf{R}^{n}$ with polynomial coefficients.

(4) For every fixed $Q \in \mathcal{U}(g), \pi_{\lambda}(Q)$ is a finite linear combination of elements in $A_{n}(\mathrm{C})$, independent of $\lambda$, whose coefficients are rational functions of $\lambda$, regular on $\Gamma$.

(5) For every $f \in L^{1}(G)$ and every $v \in H$, the map $\lambda \mapsto \pi_{\lambda}(f) v$ from $\Gamma$ to $H$ is continuous; moreover, the function $\lambda \mapsto\left\|\pi_{\lambda}(f)\right\|$ tends to 0 as $\lambda$ approaches infinity.

(6) For every $f \in L^{1}(G)$ the nonnegative (possibly $+\infty$-valued) function $\lambda \mapsto \operatorname{tr}\left(\pi_{\lambda}(f) \pi_{\lambda}(f)^{*}\right)$ on $\Gamma$ (where tr denotes trace) is lower semicontinuous. There is a real-valued rational function $R$, regular on $\Gamma$, and unique up to multiplication by -1 , such that

(2.28) For every $f \in L^{1}(G) \cap L^{2}(G)$,

$$
\int_{G}|f(x)|^{2} d x=\int_{\Gamma} \operatorname{tr}\left(\pi_{\lambda}(f) \pi_{\lambda}(f)^{*}\right) d \mu(\lambda)
$$

where $d \mu(\lambda)=\left|R\left(\lambda_{1}, \ldots, \lambda_{q}\right)\right| d \lambda_{1} \ldots d \lambda q$. 
[Note that it follows from (2.28) that $\pi_{\lambda}(f)$ is a Hilbert-Schmidt operator du-almost everywhere.]

(7) Let $K$ be the Hilbert-space of Hilbert-Schmidt operators on $H$, with the inner-product $\langle S, T\rangle=\operatorname{tr}\left(S T^{*}\right)$, and let $L^{2}(\Gamma ; K)$ denote the $L^{2}$-functions on $\Gamma$ with values in $K$, with respect to the measure $d \mu$. Then there is a unique bijective isometry $\Phi: L^{2}(G) \rightarrow L^{2}(\Gamma ; K)$ such that for every $f \in L^{1}(G) \cap L^{2}(G), \Phi(f)$ is the function $\lambda \mapsto \pi_{\lambda}(f)$.

(8) For every $\varphi \in C_{0}^{\infty}(G)$ and every $\lambda \in \Gamma, \pi_{\lambda}(\varphi)$ is of trace-class and the function $\lambda \mapsto \operatorname{tr} \pi_{\lambda}(\varphi)$ is in $L^{1}(\Gamma ; d \mu)$. Moreover,

$(2.29)\langle\delta, \varphi\rangle=\varphi(e)=\int_{\Gamma} \operatorname{tr} \pi_{\lambda}(\varphi) d \mu(\lambda)$.

Nores. 1.If $W \in Z(g)$ and $\pi$ is an irreducible unitary representation of $G$, then $\pi(Z)=c I$ for some $c \in \mathbf{C}$. By (2.12), if $W=W^{*}$, then $c \in \mathbf{R}$.

2. The polynomials on $g^{*}$ corresponding to $W_{i}, i=1, \ldots, q$, under the symmetrization map parametrize the "generic" orbits. We caution the reader that the symmetrization maps in [2] and [15] are slightly different.

3. Since $L^{1}(G) \cap L^{2}(G)$ is dense in $L^{2}(G)$ the existence of a (unique) isometric extension $\Phi$ from $L^{2}(G)$ into $L^{2}(\Gamma ; K)$ is automatic. The delicate part is to show that $\Phi$ is surjective. It would be desirable to have a simpler proof of this along the lines of the proof in [13] for the case $G=\mathbf{R}^{N}$.

4. The fact that the function $\lambda \mapsto \operatorname{tr} \pi_{\lambda}(\varphi)$ is in $L^{1}(\Gamma ; d \mu)$ follows from the treatment in [15] describing $\operatorname{tr} \pi_{\lambda}(\varphi)$ as the integral of the Fourier transform of $\varphi$ over the coadjoint orbit in $g^{*}$ corresponding to $\pi_{\lambda}$. This interpretation also indicates that we should expect much better regularity properties for $\lambda \mapsto \operatorname{tr} \pi_{\lambda}(\varphi)$ than simply measurability. We shall see later that when $G=$ $H_{n}$ this map is $C^{\infty}$.

We wish to discuss some consequences of the Plancherel theorem. We maintain the preceding notation.

LEMMA 2.8. Let $G$ be a simply-connected nilpotent Lie group. Then for any $u \in \mathcal{E}^{\prime}(G)$ and any $\varphi \in C_{0}^{\infty}(G)$,

$$
(u, \bar{\varphi})=\int_{\Gamma} \operatorname{tr}\left(\pi_{\lambda}(u) \pi_{\lambda}(\varphi)^{*}\right) d \mu(\lambda) .
$$

Proof. $(u, \bar{\varphi})=(u * \bar{\varphi})(e)=\left(u *\left(\varphi^{\#}\right)\right)(e)$, and $u *\left(\varphi^{\#}\right) \in C_{0}^{\infty}(G)$. Thus, by (2.29),

$$
(u, \bar{\varphi})=\int_{\Gamma} \operatorname{tr}\left(\pi_{\lambda}\left(u * \varphi^{\#}\right)\right) d \mu(\lambda) .
$$

Then (2.30) follows from Lemma 2.3 and from Proposition 2.6(4).

LEMMA 2.9. Let $G$ be a simply-connected nilpotent Lie group. Then for any $f$, $g \in L^{2}(G)$, 


$$
\int_{G} f(x) \overline{g(x)} d x=\langle\Phi(f), \Phi(g)\rangle,
$$

where $\langle$,$\rangle is the Hilbert-space inner-product for L^{2}(\Gamma ; K)$.

Proof. The result is immediate, for the left-hand side of (2.31) equals $\langle f, g\rangle$, the inner-product on $L^{2}(G)$, and $\Phi$ is an isometry of Hilbert-spaces.

The following proposition is crucial for $\$ 5$.

PROPOSITION 2.10. Let $G$ be a simply-connected nilpotent Lie group, and let $u$ lie in $\mathcal{E}^{\prime}(G)$. Suppose that the function $\lambda \mapsto \pi_{\lambda}(u)$ from $\Gamma$ into the space of unbounded linear operators on $H=L^{2}\left(\mathbf{R}^{n}\right)$, say with domain the space $\delta\left(\mathbf{R}^{n}\right)$ of $C^{\infty}$-vectors, is in fact a function in $L^{2}(\Gamma ; K)$. Then $u \in L^{2}(G) \cap \mathcal{E}^{\prime}(G)$.

Proof. Since $\Phi: L^{2}(G) \rightarrow L^{2}(\Gamma ; K)$ is bijective there exists $f \in L^{2}(G)$ such that $\Phi(f)$ is the function $\lambda \mapsto \pi_{\lambda}(u)$. Let $\varphi \in C_{0}^{\infty}(G)$. By Lemma 2.8,

$$
(u, \bar{\varphi})=\int_{\Gamma} \operatorname{tr}\left(\pi_{\lambda}(u) \pi_{\lambda}(\varphi)^{*}\right) d \mu(\lambda) .
$$

But $C_{0}^{\infty}(G) \subseteq L^{1}(G) \cap L^{2}(G)$, so $\Phi(\varphi)$ is the function $\lambda \mapsto \pi_{\lambda}(\varphi)$. Thus, by Lemma 2.9,

$$
\int_{G} f(x) \overline{\varphi(x)} d x=\int_{\Gamma} \operatorname{tr}\left(\pi_{\lambda}(u) \pi_{\lambda}(\varphi)^{*}\right) d \mu(\lambda)
$$

That is, $(u, \bar{\varphi})=(f, \bar{\varphi})$, where we consider $f$ as an element of $\mathscr{D}^{\prime}(G)$. Since $\varphi \in C_{0}^{\infty}(G)$ is arbitrary, $u=f$.

We next recall the notion of dilations for the simply-connected nilpotent Lie group $G$.

Definition 2.11. Let $r \mapsto \delta_{r}$ be a homomorphism from $\mathbf{R}^{+}$, the multiplicative group of positive real numbers, into Aut $g$, the group of automorphisms of $g$, of the form $\delta_{r}=\exp ((\log r) A)$, where $A: g \rightarrow g$ is a semisimple linear transformation with positive eigenvalues, $\gamma_{1}, \ldots, \gamma_{N}$. We then call $\left\{\delta_{r}\right\}$ a group of dilations for $G$.

Notes. 1. Taking an ordered basis $X_{1}, \ldots, X_{N}$ for $g$ of eigenvectors for $A$, we see that $\delta_{r}\left(X_{i}\right)=r^{\gamma_{i}} X_{i}$. We shall generally be interested in cases where all the $\gamma_{i}$ are positive integers.

2. Since $G$ is simply-connected, $\left\{\delta_{r}\right\}$, via the exponential map, forms a group of automorphisms of $G$.

3. Of course, $\left\{\delta_{r}\right\}$ also determines a group of automorphisms of $U(g)$. For various examples of nilpotent groups with dilations, see, for example, Folland [6].

DEFinItion 2.12. $P \in \mathcal{U}(g)$ is homogeneous of degree $k$ if $\delta_{r}(P)=r^{k} P$ for every $r$. Observe that if we expand $P$ as in (2.2) in terms of the basis $X_{1}, \ldots, X_{N}$ in Note 1 , then $P$ is homogeneous of degree $k$ if and only if 
$\sum_{j=1}^{N} \alpha_{j} \gamma_{j}=k$ for every $\alpha$ with $a_{\alpha} \neq 0$. Suppose now that each $W_{i}, i=$ $1, \ldots, q$, in Theorem 2.7 is homogeneous of degree $m_{i}$; that is, $\delta_{r}\left(W_{i}\right)=$ $r^{m_{i}} W_{i}$. Define "dilations" $\delta_{r}: \mathbf{R}^{q} \rightarrow \mathbf{R}^{q}$ by $\delta_{r}: \lambda=\left(\lambda_{1}, \ldots, \lambda_{q}\right) \mapsto$ $\left(r^{m_{1}} \lambda_{1}, \ldots, r^{m_{q}} \lambda_{q}\right)$. Suppose also that each $\delta_{r}: \mathbf{R}^{q} \rightarrow \mathbf{R}^{q}$ maps $\Gamma$ into $\Gamma$, and that $0 \notin \Gamma$. Clearly, for every $\lambda \in \mathbf{R}^{q}, \lambda \neq 0$, there is a unique $r \in \mathbf{R}^{+}$such that $\left\|\delta_{r-1} \lambda\right\|=1$, where \|\| denotes the Euclidean norm in $\mathbf{R}^{q}$. We call this positive number $r$ the "norm" of $\lambda$, and denote it by $|\lambda|$. (Compare with Folland [6, §1].)

Since each $\delta_{r}$ is an automorphism of $G, \pi_{\lambda} \circ \delta_{r}$ is an irreducible unitary representation of $G$ for every $\lambda \in \Gamma$. Since $\delta_{r}\left(W_{i}\right)=r^{m_{i}} W_{i}$, it follows by (1) of Theorem 2.7 that $\pi_{\lambda} \circ \delta_{r}\left(W_{i}\right)=r^{m} \lambda_{i} I$. Hence, again by (1), $\pi_{\lambda} \circ \delta_{r}$ is unitarily equivalent to $\pi_{\delta, \lambda}$. That is,

(2.32) For every $\lambda \in \Gamma, r \in \mathbf{R}^{+}$, there is a unitary operator $U_{\lambda, r}$ on $H$ such that $\pi_{\lambda} \circ \delta_{r}=U_{\lambda, r} \pi_{\delta, \lambda} U_{\lambda, r}^{-1}$. (Since $\pi_{\delta, \lambda}$ is irreducible, $U_{\lambda, r}$ is unique, up to scalar factor of modulus 1.)

In order to work effectively with homogeneous $P \in U(g)$ we must renormalize our choice of $\pi_{\lambda}$ to get rid of the $U_{\lambda, r}$.

LEMMA 2.13. For every $\lambda \in \Gamma$ there is a unitary operator $V_{\lambda}$ on $H$ such that the representations $\tilde{\pi}_{\lambda} \equiv V_{\lambda} \pi_{\lambda} V_{\lambda}^{-1}$ satisfy the same properties as the $\pi_{\lambda}$ in Theorem 2.7 (with (4) slightly modified), and, in addition, satisfy

$$
\tilde{\pi}_{\lambda} \circ \delta_{r}=\tilde{\pi}_{\delta, \lambda} \text { for every } \lambda \in \Gamma \text { and } r \in \mathbf{R}^{+} \text {. }
$$

Proof. A simple computation shows that to ensure (2.33) it suffices that

$$
V_{\delta_{r} \lambda} \cong V_{\lambda} U_{\lambda, r}
$$

where $\cong$ denotes equality up to scalar multiple of modulus 1 . But it follows from (2.32) that $U_{\lambda, r}$ satisfies the "cocycle" condition

$$
U_{\lambda, r_{1}} \circ U_{\delta_{1}, \lambda_{1}, r_{2}} \cong U_{\lambda, r_{1} r_{2}} \text { for every } \lambda \in \Gamma, r_{1}, r_{2} \in \mathbf{R}^{+} \text {. }
$$

If we now define $V_{\lambda}$ by

$$
V_{\lambda}=U_{\delta_{\mu \mathrm{p}}-1 \lambda,|\lambda|},
$$

it follows from (2.35) and the definition of $|\lambda|$ that (2.34) holds, i.e., the "cocycle" $U_{\lambda, r}$ is a "coboundary". Thus (2.33) holds.

It follows from (2.32) that for any $v \in H=L^{2}\left(\mathrm{R}^{n}\right), v$ is a $C^{\infty}$-vector for $\pi_{\delta, \lambda}$ if and only if $U_{\lambda, r} v$ is a $C^{\infty}$-vector for $\pi_{\lambda} \circ \delta_{r}$. But by Theorem 2.7(2), for each of these representations the space of $C^{\infty}$-vectors is $\delta\left(\mathbf{R}^{n}\right)$. Thus, for any $\lambda, r$, and any $v \in L^{2}\left(\mathbf{R}^{n}\right), U_{\lambda, r} v$ is in $\delta\left(\mathbf{R}^{n}\right)$ if and only if $v$ is in $\delta\left(\mathbf{R}^{n}\right)$. Hence, by the definition, (2.36), of $V_{\lambda}$, it follows that the space of $C^{\infty}$-vectors for $\tilde{\pi}_{\lambda}$ is $\delta\left(\mathbf{R}^{n}\right)$. Thus (2) holds for $\tilde{\pi}_{\lambda}$.

From Theorem 2.7(3) and (2.32) it follows that for every $\lambda, r$,

$$
A_{n}(\mathrm{C})=\left\{U_{\lambda, r} T U_{\lambda, r}^{-1} \mid T \in A_{n}(\mathrm{C})\right\}
$$


Hence, again by (2.36), it follows that (3) holds for $\tilde{\pi}_{\lambda}$.

Now let $Q$ be an element of $\mathscr{Q}(g)$. By Theorem 2.7(4) there exist $T_{1}, \ldots, T_{k} \in A_{n}(\mathrm{C})$ and rational functions $R_{1}(\lambda), \ldots, R_{k}(\lambda)$ such that

$$
\pi_{\lambda}(Q)=\sum_{j=1}^{k} R_{j}(\lambda) T_{j}
$$

If $Q$ is homogeneous of degree $m$ it follows that

$$
\pi_{\lambda} \circ \delta_{r}(Q)=r^{m} \sum_{j=1}^{k} R_{j}(\lambda) T_{j}
$$

But by (2.32) and (2.38),

$$
\pi_{\lambda} \circ \delta_{r}(Q)=U_{\lambda, r}\left(\sum_{j=1}^{k} R_{j}\left(\delta_{r} \lambda\right) T_{j}\right) U_{\lambda, r}^{-1}
$$

Thus the right-hand sides of (2.39) and (2.40) are equal for any $\lambda, r$. Now replace $\lambda$ by $\delta_{|\lambda|-1} \lambda$ and $r$ by $|\lambda|$; of course this replaces $\delta_{r} \lambda$ by $\lambda$. Hence by (2.36) and the above we get

$$
V_{\lambda}\left(\sum_{j=1}^{k} R_{j}(\lambda) T_{j}\right) V_{\lambda}^{-1}=|\lambda|^{m} \sum_{j=1}^{k} R_{j}\left(\delta_{|\lambda|}-1 \lambda\right) T_{j}
$$

But by (2.38) the left-hand side of (2.41) equals $\tilde{\pi}_{\lambda}(Q)$. We have therefore shown

(2.42) If $Q \in \mathcal{U}(\mathrm{g})$ satisfies (2.38) and is homogeneous of degree $m$, then

$$
\tilde{\pi}_{\lambda}(Q)=|\lambda|^{m} \sum_{j=1}^{k} R_{j}\left(\delta_{|\lambda|^{-1}} \lambda\right) T_{j}
$$

Clearly, every $Q \in \mathcal{U}(g)$ is uniquely expressible as a finite sum of homogeneous elements of $U(g)$. Applying (2.42) to each of these separately we obtain the desired analogue of Theorem 2.7(4).

Just as in Dixmier [2; see, in particular, Lemma 33], one shows by applying (4) to $Q \in g$ that for any $v \in H$ the map $(x, \lambda) \mapsto \tilde{\pi}_{\lambda}(x) v$ from $G \times \Gamma$ into $H$ is continuous, and the first part of (5) follows. Properties (1), (6), (8), and the second part of (5) are clearly invariant under unitary equivalence. Property (7) for $\tilde{\pi}_{\lambda}$ is proved the same way as for $\pi_{\lambda}$. This concludes the proof of the lemma.

Condition (2.33) allows us to pass to the unit ball in $\mathbf{R}^{q}$ when dealing with homogeneous operators. More precisely,

(2.43) If $P \in Q(g)$ is homogeneous of degree $m$, then for any $\lambda \in \Gamma$,

$$
\tilde{\pi}_{\lambda}(P)=|\lambda|^{m} \tilde{\pi}_{\delta_{|\lambda|-1 \lambda}}(P)=|\lambda|^{m} \pi_{\delta_{|\lambda|-1 \lambda}}(P) .
$$

Proof. The first equality follows immediately from (2.33). Since $\delta_{1}$ is the identity map it follows from (2.32) that $U_{\lambda, 1} \simeq I$ for any $\lambda \in \Gamma$. Hence, by 
(2.36), $V_{\lambda} \cong I$ if $|\lambda|=1$. Thus $\tilde{\pi}_{\lambda}=\pi_{\lambda}$ if $|\lambda|=1$, and so the second equality holds.

We shall need to work interchangeably with $P$ and $\bar{P}$ (resp., $P^{*}$ and $P^{t}$ ). We therefore introduce the following considerations. Suppose that for every $i=1, \ldots, q W_{i}$ is either symmetric, i.e., $W_{i}=W_{i}^{t}$, or antisymmetric, i.e., $W_{i}=-W_{i}^{t}$, and let $\tau_{i}=1$ or -1 , correspondingly. (Since each $W_{i}$ is selfadjoint, i.e., $W_{i}=W_{i}^{*}$, these conditions correspond to $\bar{W}_{i}=W_{i}, \bar{W}_{i}=$ $-W_{i}$, respectively.) Also, for any $\lambda \in \mathbf{R}^{q}$, let $\tau \lambda=\left(\tau_{1} \lambda, \ldots, \tau_{q} \lambda_{q}\right)$, and suppose that $\tau$ leaves $\Gamma$ invariant.

Since $\tilde{\pi}_{\lambda}$ is an irreducible unitary representation of $G$, so is $\hat{\pi}_{\lambda}$, defined by $\hat{\pi}_{\lambda}(x)=\left[\tilde{\pi}_{\lambda}\left(x^{-1}\right)\right]^{t}=\overline{\pi_{\lambda}(x)}$, where ${ }^{t}$ denotes the Hilbert-space transpose (rather than adjoint) in $L^{2}\left(\mathbf{R}^{n}\right)$, and where $\overline{\pi_{\lambda}(x)} v=\left(\pi_{\lambda}(x) v\right)^{-}$for any $v \in L^{2}\left(\mathbf{R}^{n}\right)$. Since $\delta\left(\mathbf{R}^{n}\right)$ is closed with respect to ${ }^{-}$, it follows from the second equality above that the space of $C^{\infty}$-vectors for $\hat{\pi}_{\lambda}$ is also $\delta\left(\mathbf{R}^{n}\right)$.

At the level of $U(g), \hat{\pi}_{\lambda}$ takes the form

$$
\hat{\pi}_{\lambda}(Q)=\left[\tilde{\pi}_{\lambda}\left(Q^{t}\right)\right]^{t}=\left[\tilde{\pi}_{\lambda}(\bar{Q})\right]^{-},
$$

the second equality follows from (2.12) and the fact that $\bar{Q}=Q^{t *}$. It follows from Theorem 2.7 (1) that $\hat{\pi}_{\lambda}$ is unitarily equivalent to $\tilde{\pi}_{\tau \lambda}$. If $S_{\lambda}$ is the (unique up to scalar multiple) unitary operator on $L^{2}\left(\mathbf{R}^{n}\right)$ implementing the equivalence, then $S_{\lambda}$ and $S_{\lambda}^{-1}$ map $C^{\infty}$-vectors to $C^{\infty}$-vectors, i.e., $S_{\lambda}$ and $S_{\lambda}^{-1}$ map $\delta\left(\mathbf{R}^{n}\right)$ to $\delta\left(\mathbf{R}^{n}\right)$. Viewing the equivalence at the level of $U(g)$ we see that for every $Q \in \mathcal{U}(g)$,

$$
\left[\tilde{\pi}_{\lambda}(\bar{Q})\right]^{-}=\left[\tilde{\pi}_{\lambda}\left(Q^{t}\right)\right]^{t}=S_{\lambda} \tilde{\pi}_{\tau \lambda}(Q) S_{\lambda}^{-1} \text { for every } \lambda \in \Gamma .
$$

Since $S_{\lambda}, S_{\lambda}^{-1}$ map $\delta\left(\mathbf{R}^{n}\right)$ to $\delta\left(\mathbf{R}^{n}\right)$, it follows from (2.45) that if $\tilde{\pi}_{\lambda}(P)$ (resp. $\left.\tilde{\pi}_{\lambda}\left(P^{*}\right)\right)$ has a bounded left or right inverse in the sense of Definition 3.2 for every $\lambda \in \Gamma$, then so does $\tilde{\pi}_{\lambda}(\bar{P})$ (resp. $\tilde{\pi}_{\lambda}\left(P^{t}\right)$ ).

Finally we note that if $P$ is homogeneous of degree $k$, then the same is true of $\bar{P}, P^{*}, P^{t}$. This follows, for example, from the remark immediately following Definition 2.12 and from (2.4)-(2.6).

3. Construction of the parametrix. Recall that the $2 n+1$-dimensional Heisenberg algebra, $h_{n}$, is the Lie algebra with generators $X_{i}, i=1, \ldots, n, Y_{i}$, $i=1, \ldots, n, Z$ satisfying the commutation relations

$$
\left[X_{i}, Y_{j}\right]=\delta_{i j} Z, \quad\left[X_{i}, Z\right]=0, \quad\left[Y_{i}, Z\right]=0 .
$$

The Heisenberg group $H_{n}$ is the unique simply-connected Lie group having $h_{n}$ as its Lie algebra. The group $H_{n}$ has a group of dilations $\left\{\delta_{r}\right\}$ defined by

$$
\delta_{r}\left(X_{i}\right)=r X_{i}, \quad \delta_{r}\left(Y_{i}\right)=r Y_{i}, \quad \delta_{r}(Z)=r^{2} Z .
$$

In this section we want to prove the following: 
THEOREM 3.1. Let $P \in \mathcal{U}\left(h_{n}\right)$ be homogeneous, and suppose

(3.3) $\pi(P)$ has a bounded right-inverse for every irreducible unitary representation $\pi$ of $H_{n}$ (except the trivial, identity representation).

Then $P$ has a right parametrix. That is, there exists $u \in \mathscr{D}^{\prime}(G)$ and $f \in$ $C_{0}^{\infty}(G)$ such that

$$
P u=\delta+f .
$$

As in $\$ 2$ we let $\mathrm{H}$ denote the Hilbert-space of $\pi$, and $\mathrm{H}_{\infty}$ the space of $\mathrm{C}^{\infty}$-vectors.

Definition 3.2. A bounded right (resp., left) inverse for $\pi(P)$ is a bounded linear operator $B: H \rightarrow H$ such that

(3.5) $B$ maps $H_{\infty}$ into $H_{\infty}$,

(3.6) $\pi(P) B=I$ on $H_{\infty}$ (resp., $B \pi(P)=I$ on $\left.H_{\infty}\right)$.

We begin with a discussion of the irreducible unitary representations of $H_{n}$. (See, for example, [2], [3], [15]. We shall adhere most closely to the treatment in [15]) We shall use exponential coordinates, $(a, b, c) \in \mathbf{R}^{2 n+1} \mapsto \exp (a \cdot X$ $+b \cdot Y+c Z)$, on $H_{n}$.

There are two classes of irreducible unitary representations, as follows from the Stone-von Neumann theorem:

(1) A "degenerate" family of 1-dimensional representations which map $Z$ to 0 . These correspond to the irreducible unitary representations of $H_{n} /\{\exp c Z \mid c \in \mathbf{R}\} \cong \mathbf{R}^{2 n}$. They are parametrized by $(\xi, \eta) \in \mathbf{R}^{2 n}$, and are given by

$$
\pi_{(\xi, \eta)}(a, b, c)=e^{i(a \cdot \xi+b, \eta)}, \quad(\xi, \eta) \in \mathbf{R}^{2 n} .
$$

The trivial 1-dimensional identity representation corresponds to $(\xi, \eta)=0$. At the Lie algebra level these representations take the form

$$
\pi_{(\xi, \eta)}\left(X_{i}\right)=\sqrt{-1} \xi_{i}, \quad \pi_{(\xi, \eta)}\left(Y_{i}\right)=\sqrt{-1} \eta_{i}, \quad \pi_{(\xi, \eta)}(Z)=0 .
$$

(2) A "generic" family parametrized by $\lambda \in \mathbf{R}-\{0\}$, acting on $L^{2}\left(\mathbf{R}^{n}\right)$, which map $Z$ to a nonzero scalar. They are given by

$$
\left[\pi_{\lambda}(a, b, c) v\right](t)=e^{i \lambda(b \cdot t+c+a \cdot b / 2)} v(t+a), \text { for } v \in L^{2}\left(\mathbf{R}^{n}\right) .
$$

The space of $C^{\infty}$-vectors for each $\pi_{\lambda}$ is $\delta\left(\mathbf{R}^{n}\right)$. At the Lie algebra level these representations take the form

$$
\pi_{\lambda}\left(X_{i}\right)=d / d t_{i}, \quad \pi_{\lambda}\left(Y_{i}\right)=\sqrt{-1} \lambda t_{i}, \quad \pi_{\lambda}(Z)=\sqrt{-1} \lambda .
$$

This family of representations, $\left\{\pi_{\lambda}\right\}$, is the one occurring in Theorem 2.7. Indeed, when $G=H_{n}, Z(g)$ consists precisely of the polynomials in $Z$, so $q=1$, and we can take $W=Z / \sqrt{-1} . \Gamma$ becomes $\mathbf{R}-\{0\}$, and, as seen in [3], the Plancherel measure $d \mu(\lambda)$ equals $|\lambda|^{n} d \lambda$, where || denotes absolute value.

Since $Z$ is homogeneous of degree 2 , it follows that $|\lambda|=|\lambda|^{1 / 2}$ for every 
$\lambda \in \mathbf{R}-\{0\}$. Thus, when we renormalize as in Lemma 2.13 , it follows from (2.43) and (3.10) that

$$
\begin{aligned}
\tilde{\pi}_{\lambda}\left(X_{i}\right)=|\lambda|^{1 / 2} \frac{d}{d t_{i}}, \quad \tilde{\pi}_{\lambda}\left(Y_{i}\right)=\sqrt{-1}(\operatorname{sgn} \lambda)|\lambda|^{1 / 2} t_{i}, \\
\tilde{\pi}_{\lambda}(Z)=\sqrt{-1} \lambda .
\end{aligned}
$$

More generally it follows from (2.43) that

(3.12) If $P \in \mathcal{U}\left(h_{n}\right)$ is homogeneous of degree $m$, then

$$
\tilde{\pi}_{\lambda}(P)=|\lambda|^{m / 2} \tilde{\pi}_{1}(P)=|\lambda|^{m / 2} \pi_{1}(P) \text { if } \lambda>0,
$$

and

$$
\tilde{\pi}_{\lambda}(P)=|\lambda|^{m / 2} \tilde{\pi}_{-1}(P)=|\lambda|^{m / 2} \pi_{-1}(P) \quad \text { if } \lambda<0 .
$$

The unitary operator $V_{\lambda}$ implementing the equivalence between $\tilde{\pi}_{\lambda}$ and $\pi_{\lambda}$ is given by $v(t) \mapsto|\lambda|^{-n / 4} v\left(|\lambda|^{-1 / 2} t\right)$ for $v \in L^{2}\left(\mathrm{R}^{2 n}\right)$.

We also have from (3.8) that $\pi_{(\xi, \eta)} \circ \delta_{r}=\pi_{(r, m)}$. Hence,

(3.13) If $P \in \mathcal{Q}\left(h_{n}\right)$ is homogeneous of degree $m$, then for any $r \in \mathbf{R}^{+}$and any $(\xi, \eta) \in \mathbf{R}^{2 n}$,

$$
\pi_{(r \xi, \eta \eta)}(P)=r^{m} \pi_{(\xi, \eta)}(P) .
$$

Since $W=-W^{t}$, we can apply the considerations at the end of $\$ 2$. In fact, a straightforward verification shows that we can take $S_{\lambda}$ in (2.45) equal to $I$. Hence (2.45) takes the form

$$
\tilde{\pi}_{\lambda}\left(Q^{t}\right)=\left[\tilde{\pi}_{-\lambda}(Q)\right]^{t}, \quad \tilde{\pi}_{\lambda}(\bar{Q})=\left[\tilde{\pi}_{-\lambda}(Q)\right]^{-}
$$

for every $\lambda \in \mathbf{R}-\{0\}$. Similarly we see

$$
\pi_{(\xi, \eta)}\left(Q^{t}\right)=\pi_{(-\xi,-\eta)}(Q), \quad \pi_{(\xi, \eta)}(\bar{Q})=\left[\pi_{(-\xi,-\eta)}(Q)\right]^{-}
$$

for every $(\xi, \eta) \in \mathbf{R}^{2 n}$.

Remarks. 1. For $\varphi \in C_{0}^{\infty}\left(H_{n}\right)$, let $K(\varphi ; \lambda, s, t)$ denote the kernel associated to $\pi_{\lambda}(\varphi)$, and $\tilde{K}(\varphi ; \lambda, s, t)$ the kernel associated to $\tilde{\pi}_{\lambda}(\varphi)$. It follows from (3.9) that

$$
\begin{aligned}
K(\varphi ; \lambda, s, t) & =\int e^{i \lambda(b \cdot s+c+(t-s) \cdot b / 2)} \varphi(t-s, b, c) d b d c \\
& =\tilde{\varphi}(t-s, \lambda((s+t) / 2), \lambda),
\end{aligned}
$$

where $\tilde{\varphi}$ here denotes the (inverse) partial Fourier transform of $\varphi(a, b, c)$ with respect to the variables $b, c$. (We ignore factors of $2 \pi$.) Also, it follows from the explicit form of $V_{\lambda}$ that

$$
\tilde{K}(\varphi ; \lambda, s, t)=|\lambda|^{-n / 2} K\left(\varphi, \lambda,|\lambda|^{-1 / 2} s,|\lambda|^{-1 / 2} t\right) .
$$

Notice that $K(\varphi ; \lambda, s, t)$, originally defined only for $\lambda \in \mathbf{R}-\{0\}$ can, by the Paley-Wiener theorem, be viewed as defined for all $\lambda \in \mathbf{C}, C^{\infty}$ in $(\lambda, s, t)$, 
holomorphic in $\lambda$ for fixed $s, t$, and having a well-determined rate of growth at $\infty$.

2. Using integration by parts and differentiation under the integral the following can be proved:

(3.18) For every $\varphi \in C_{0}^{\infty}\left(H_{n}\right)$ there exists a unique $\psi \in C_{0}^{\infty}\left(H_{n}\right)$ such that

$$
\frac{d}{d \lambda} K(\varphi ; \lambda, s, t)=\lambda^{-1} K(\psi ; \lambda, s, t), \quad \lambda \neq 0 .
$$

(3.19) For every $\varphi \in C_{0}^{\infty}\left(H_{n}\right)$ there exists a unique $\eta \in C_{0}^{\infty}\left(H_{n}\right)$ such that

$$
\frac{d}{d \lambda} \tilde{K}(\varphi ; \lambda, s, t)=\lambda^{-1} \tilde{K}(\eta ; \lambda, s, t), \quad \lambda \in \mathbf{R}-\{0\} .
$$

The functions $\psi, \eta$ can be explicitly expressed in terms of $\varphi$.

3. Using either (3.16) or the general orbit picture in [15] we find

$$
\operatorname{tr} \tilde{\pi}_{\lambda}(\varphi)=\operatorname{tr} \pi_{\lambda}(\varphi)=\hat{\varphi}(0,0, \lambda) /|\lambda|^{n}
$$

where

$\hat{\varphi}$ denotes the (inverse) partial Fourier transform of $\varphi(a, b, c)$ with respect to the variable $c$.

We next sketch the idea for the construction of a parametrix for $P \in$ थ $\left(h_{n}\right)$, homogeneous of degree $m$, and satisfying (3.3). Let $B_{1}, B_{-1}$ be bounded right-inverses for $\tilde{\pi}_{1}(P), \tilde{\pi}_{-1}(P)$, respectively. Then if the integral

$$
(u, \varphi)=\int_{\mathbf{R}-\{0\}} \operatorname{tr}\left(\tilde{\pi}_{\lambda}(\varphi) \tilde{\pi}_{\lambda}(P)^{-1}\right) d \mu(\lambda)
$$

were well defined for $\varphi \in C_{0}^{\infty}\left(H_{n}\right)$, and determined a distribution $u$, then $u$ would satisfy the equation $P u=\delta$. Indeed, we would have

$$
\begin{aligned}
(P u, \varphi) & =\left(u, P^{\prime} \varphi\right)=\int_{\mathbf{R}-\{0\}} \operatorname{tr}\left(\tilde{\pi}_{\lambda}\left(P^{\prime} \varphi\right) \tilde{\pi}_{\lambda}(P)^{-1}\right) d \mu(\lambda) \\
& =\int_{\mathbf{R}-\{0\}} \operatorname{tr}\left(\tilde{\pi}_{\lambda}(\varphi) \tilde{\pi}_{\lambda}(P) \tilde{\pi}_{\lambda}(P)^{-1}\right) d \mu(\lambda) \quad(\text { by }(2.13)) \\
& =\int_{\mathbf{R}-\{0\}} \operatorname{tr}\left(\tilde{\pi}_{\lambda}(\varphi)\right) d \mu(\lambda) \\
& =(\delta, \varphi) \quad(\text { by }(2.29)) .
\end{aligned}
$$

But, by (3.12),

$$
\begin{aligned}
\tilde{\pi}_{\lambda}(P)^{-1} & =|\lambda|^{-m / 2} B_{1} \text { if } \lambda>0 ; \\
\tilde{\pi}_{\lambda}(P)^{-1} & =|\lambda|^{-m / 2} B_{-1} \text { if } \lambda<0 .
\end{aligned}
$$

Thus, since $d \mu(\lambda)=|\lambda|^{n} d \lambda$, 


$$
\begin{aligned}
\int_{\mathbf{R}-\{0\}} \operatorname{tr}\left(\tilde{\pi}_{\lambda}(\varphi) \tilde{\pi}_{\lambda}(P)^{-1}\right) d \mu(\lambda)= & \int_{\mathbf{R}^{+}}|\lambda|^{-m / 2} \operatorname{tr}\left(\tilde{\pi}_{\lambda}(\varphi) B_{1}\right)|\lambda|^{n} d \lambda \\
& +\int_{\mathbf{R}^{-}}|\lambda|^{-m / 2} \operatorname{tr}\left(\tilde{\pi}_{\lambda}(\varphi) B_{-1}\right)|\lambda|^{n} d \lambda .
\end{aligned}
$$

We shall see that both the integrands behave well for $|\lambda|$ large, but clearly they may blow up as $|\lambda| \rightarrow 0$. (See, for instance, (3.20).) Thus $u$ in (3.21) is not well defined. We will, however, be able to solve the equation $P v=[P \bar{P}+$ $\left.Z^{2 m}\right] \delta$ with the Plancherel formula. Moreover, we shall see that, since $\pi_{(\xi, \eta)}(P) \neq 0$ for $(\xi, \eta) \neq 0, P \bar{P}+Z^{2 m}$ is elliptic. We can consequently obtain a solution $u$ of (3.4) be convolving $v$ with a compactly-supported parametrix for $P \bar{P}+Z^{2 m}$.

We proceed next to the details. First, in order to estimate $\operatorname{tr}\left(\tilde{\pi}_{\lambda}(\varphi) B_{1}\right)$ and $\operatorname{tr}\left(\tilde{\pi}_{\lambda}(\varphi) B_{-1}\right)$ without introducing the trace-norm of $\pi_{\lambda}(\varphi)$, which does not seem to us to be simply-expressible in terms of $\operatorname{tr}\left(\tilde{\pi}_{\lambda}(\varphi)\right)$ or $\operatorname{tr} \tilde{\pi}_{\lambda}\left(\varphi * \varphi^{\#}\right)$, we would like $B_{1}$ and $B_{-1}$ to be Hilbert-Schmidt, or even of trace class, rather than merely bounded. We proceed by replacing $P$ by $P Q$, where $Q$ is a homogeneous operator in $\mathcal{U}\left(h_{n}\right)$ satisfying (3.3), and such that the bounded right-inverses $\tilde{\pi}_{1}(Q)^{-1}, \tilde{\pi}_{-1}(Q)^{-1}$ are of trace-class. Then $\tilde{\pi}_{1}(Q)^{-1} B_{1}$, $\tilde{\pi}_{-1}(Q)^{-1} B_{-1}$ are bounded right-inverses for $\tilde{\pi}_{1}(P Q), \tilde{\pi}_{-1}(P Q)$, and are of trace-class, since the trace-class operators form a two-sided ideal in the space of bounded operators. Notice, moreover, that if $u$ is a parametrix for $P Q$, then $Q u$ is a parametrix for $P$; also, if $u$ is $C^{\infty}$ away from $e$, so is $Q u$. Actually, as will follow from $\S 4$, we could take $Q=P^{N}$ for $N$ sufficiently large. However, we would still require the following lemma.

LEMma 3.3. There exists a homogeneous $Q \in \mathcal{Q}\left(h_{n}\right)$ such that for every irreducible unitary representation $\pi$ of $H_{n}$ except $\pi_{(0,0)}, \pi(Q)$ has a bounded two-sided inverse which is, in fact, of trace-class. We can take $Q=\left[\sum_{i-1}^{n}\left(X_{i}^{2}\right.\right.$ $\left.\left.+Y_{i}^{2}\right)\right]^{N}$, for $N$ a sufficiently large positive integer depending on $n$.

Proof. Clearly $\sum_{i=1}^{n}\left(X_{i}^{2}+Y_{i}^{2}\right)$ is homogeneous of degree 2. By (3.8),

$$
\pi_{(\xi, \eta)}\left(\sum_{i=1}^{n}\left(X_{i}^{2}+Y_{i}^{2}\right)\right)=-\sum_{i=1}^{n}\left(\xi_{i}^{2}+\eta_{i}^{2}\right) \neq 0 \quad \text { if }(\xi, \eta) \neq(0,0) .
$$

By (3.10),

$$
\tilde{\pi}_{1}\left(\sum_{i=1}^{n}\left(X_{i}^{2}+Y_{i}^{2}\right)\right)=\tilde{\pi}_{-1}\left(\sum_{i=1}^{n}\left(X_{i}^{2}+Y_{i}^{2}\right)\right)=-\sum_{i=1}^{n}\left(D_{t_{i}}^{2}+t_{i}^{2}\right),
$$

where $D_{t_{4}}=(-1)^{-1 / 2}\left(d / d t_{i}\right)$. But it is well known (see, for example, [17]) that there is a complete orthonormal basis for $L^{2}\left(\mathbf{R}^{1}\right)$ consisting of eigenfunctions $\left\{v_{i}\right\}_{i=0}^{\infty}$ for the harmonic oscillator $D_{t}^{2}+t^{2}$. These so-called Hermite functions lie in $\delta\left(\mathbf{R}^{1}\right)$ and satisfy the equation 


$$
\left(D_{t}^{2}+t^{2}\right) v_{i}=(2 i+1) v_{i} \text { for every } i .
$$

It follows that the functions $v_{i}(t) \equiv v_{i_{1}}\left(t_{1}\right) \ldots v_{i_{n}}\left(t_{n}\right)$, as $i=\left(i_{1}, \ldots, i_{n}\right)$ runs through all $n$-tuples of nonnegative integers, form a complete orthonormal basis for $L^{2}\left(\mathbf{R}^{n}\right)$, and that

$$
\left[\sum_{j=1}^{n}\left(D_{t_{j}}^{2}+t_{j}^{2}\right)\right] v_{i}=\left[\sum_{j=1}^{n}\left(2 i_{j}+1\right)\right] v_{i} \text { for every } i .
$$

Thus $\sum_{j=1}^{n}\left(D_{t_{j}}^{2}+t_{j}^{2}\right)$ has a two-sided bounded inverse $T$, given by

$$
T v_{i}=\frac{1}{\sum_{j=1}^{n}\left(2 i_{j}+1\right)} v_{i}
$$

(We shall see in $\S 4$ that $T$ maps $\delta\left(\mathbf{R}^{n}\right)$ to $\delta\left(\mathbf{R}^{n}\right)$.) Since all the eigenvalues of $T$ are positive, $T^{N}$ is of trace-class precisely if

$$
\sum_{i} \frac{1}{\left[\sum_{j=1}^{n}\left(2 i_{j}+1\right)\right]^{N}}<+\infty
$$

But (3.28) is certainly equivalent to

$$
\sum_{k=1}^{\infty} \frac{\alpha(k)}{k^{N}}<+\infty
$$

where $\alpha(k)$ is the number of ways $k$ can be written as a sum of $n$ positive integers. Of course, $\alpha(k)$ can be determined explicitly; for us, however, it is enough to notice the obvious fact that $\alpha(k)<k^{n}$. Hence (3.29), and therefore (3.28), holds if $N-n>1$, i.e. if $N>n+1$.

We next examine the question of measurability of the functions $\lambda \mapsto$ $\operatorname{tr}\left(\tilde{\pi}_{\lambda}(\varphi) B_{1}\right), \lambda \mapsto \operatorname{tr}\left(\tilde{\pi}_{\lambda}(\varphi) B_{-1}\right)$. It is convenient to work in the general context of $\$ 2$. We maintain the notation of that section.

LEMMA 3.4. Let $G$ be a simply-connected nilpotent Lie group (with dilations) and let $B: H \rightarrow H$ be a bounded linear operator. Then for any $\varphi \in C_{0}^{\infty}(G)$ the function $\lambda \mapsto \operatorname{tr}\left(\tilde{\pi}_{\lambda}(\varphi) B\right)$ from $\Gamma$ to $\mathbf{C}$ is measurable. If $B$ is of trace-class then the function is in fact continuous.

Proof. First suppose only that $B$ is bounded. Since $\tilde{\pi}_{\lambda}(\varphi)$ is of trace-class so is $\tilde{\pi}_{\lambda}(\varphi) B$. Also, $\operatorname{tr}\left(\tilde{\pi}_{\lambda}(\varphi) B\right)=\operatorname{tr}\left(B \tilde{\pi}_{\lambda}(\varphi)\right)$. Let $\left\{v_{i}\right\}_{i=0}^{\infty}$ be any orthonormal basis for $H$. Then

$$
\begin{aligned}
\operatorname{tr}\left(\tilde{\pi}_{\lambda}(\varphi) B\right) & =\operatorname{tr}\left(B \tilde{\pi}_{\lambda}(\varphi)\right)=\sum_{i=0}^{\infty}\left\langle B \tilde{\pi}_{\lambda}(\varphi) v_{i}, v_{i}\right\rangle \\
& =\sum_{i=0}^{\infty}\left\langle\tilde{\pi}_{\lambda}(\varphi) v_{i}, B^{*} v_{i}\right\rangle .
\end{aligned}
$$

By Theorem 2.7(5), for each $i$ the function $\lambda \mapsto\left\langle\tilde{\pi}_{\lambda}(\varphi) v_{i}, B^{*} v_{i}\right\rangle$ from $\Gamma$ to $\mathbf{C}$ 
is continuous, a fortiori measurable. Hence $\operatorname{tr}\left(\tilde{\pi}_{\lambda}(\varphi) B\right)$, being a pointwise limit of measurable functions, is measurable.

Suppose next that $B$ is of trace-class. We express $B^{*}$ in polarized form, $B^{*}=U T$, where $T=\left(B B^{*}\right)^{1 / 2}$ and $U$ is a partial isometry. $T$ is positive definite and of trace-class, in particular, compact, so $H$ has an orthonormal basis $\left\{v_{i}\right\}_{i=0}^{\infty}$ consisting of eigenvectors of $T$, with eigenvalues $\left\{t_{i}\right\}_{i=0}^{\infty}$. (Recall that the trace-norm of $B$ is, in fact, $\sum_{i=0}^{\infty} t_{i}<\infty$.) Then

$$
\left\langle\tilde{\pi}_{\lambda}(\varphi) v_{i}, B^{*} v_{i}\right\rangle=\left\langle U^{*} \tilde{\pi}_{\lambda}(\varphi) v_{i}, T v_{i}\right\rangle=t_{i}\left\langle U^{*} \tilde{\pi}_{\lambda}(\varphi) v_{i}, v_{i}\right\rangle .
$$

In particular,

$$
\begin{aligned}
\left\langle\tilde{\pi}_{\lambda}(\varphi) v_{i}, B^{*} v_{i}\right\rangle \mid & =t_{i}\left|\left\langle U^{*} \tilde{\pi}_{\lambda}(\varphi) v_{i}, v_{i}\right\rangle\right| \\
& <t_{i}\|U\|\left\|\tilde{\pi}_{\lambda}(\varphi)\right\|=t_{i}\left\|\tilde{\pi}_{\lambda}(\varphi)\right\| \\
& <t_{i}\|\varphi\|_{L^{\prime}(G)} \quad(\text { by }(2.10)) .
\end{aligned}
$$

Thus, for every integer $n \geqslant 0$,

$$
\sum_{i=n}^{\infty}\left|\left\langle\tilde{\pi}_{\lambda}(\varphi) v_{i}, B^{*} v_{i}\right\rangle\right|<\left(\sum_{i=n}^{\infty} t_{i}\right) \cdot\|\varphi\|_{L^{\prime}(G)}
$$

Since the right-hand side of (3.33) is independent of $\lambda$, and since $\Sigma_{i=0}^{\infty} t_{i}<\infty$, the series in (3.30) converges uniformly in $\lambda$, in particular, uniformly on compact subsets of $\Gamma$. Since each of the functions $\lambda \mapsto\left\langle\tilde{\pi}_{\lambda}(\varphi) v_{i}, B^{*} v_{i}\right\rangle$ is continuous, it follows that the sum is continuous.

REMARK. Returning to the special case of $G=H_{n}$, it can be shown, using (3.19), that differentiation under the integral is valid, and that for any $v$, $w \in L^{2}\left(\mathbf{R}^{n}\right)$ the function $\lambda \mapsto\left\langle\tilde{\pi}_{\lambda}(\varphi) v, w\right\rangle$ is differentiable, and that

$$
\frac{d}{d \lambda}\left\langle\tilde{\pi}_{\lambda}(\varphi) v, w\right\rangle=\lambda^{-1}\left\langle\tilde{\pi}_{\lambda}(\eta) v, w\right\rangle \text {. }
$$

Combining this with the above procedure and iterating, we find that if $B$ is of trace-class then the function $\lambda \mapsto \operatorname{tr}\left(\tilde{\pi}_{\lambda}(\varphi) B\right)$ is $C^{\infty}$. I suspect that this is probably true for general $G$.

We are now ready to examine the convergence of integrals of the type occurring in (3.24). We begin by noting:

(3.35) For any Hilbert-Schmidt operators $S, T$ on $H,|\operatorname{tr}(S T)|<$ $\left[\operatorname{tr}\left(S S^{*}\right)\right]^{1 / 2}\left[\operatorname{tr}\left(T T^{*}\right)\right]^{1 / 2}$. This is simply a variant of the Cauchy-Schwarz inequality for the Hilbert space of Hilbert-Schmidt operators, under the inner product $\langle S, T\rangle=\operatorname{tr}\left(S T^{*}\right)$.

We now work in the context of $G=H_{n}$.

LEMMA 3.5. Let $\varepsilon>0$, and let $B$ be a trace-class operator on $L^{2}\left(\mathbf{R}^{n}\right)$. If $s \in \mathrm{C}$ with $\operatorname{Re} s>-(n+1) / 2$, then the linear map $u: C_{0}^{\infty}\left(H_{n}\right) \rightarrow \mathrm{C}$ given by

$$
(u, \varphi)=\int_{0<\lambda<\varepsilon}|\lambda|^{s+n} \operatorname{tr}\left(\tilde{\pi}_{\lambda}(\varphi) B\right) d \lambda
$$


is well defined, and $u \in \mathscr{D}^{\prime}\left(H_{n}\right)$. In fact, there exists a constant $C$ independent of $\varphi$ such that

$$
|(u, \varphi)|<\int_{0<\lambda<\varepsilon}|\lambda|^{\operatorname{Re} s+n}\left|\operatorname{tr}\left(\tilde{\pi}_{\lambda}(\varphi) B\right)\right| d \lambda<C\|\varphi\|_{L^{2}\left(H_{n}\right)}
$$

so $u \in L^{2}\left(H_{n}\right)$. The same is true if we replace the domain of integration by $-\varepsilon<\lambda<0$.

Proof. By the preceding lemma, the integrand in (3.36) is measurable, in fact $C^{\infty}$. To show that it is integrable, i.e., in $L^{1}$, observe that

$$
|\lambda|^{\operatorname{Res}+n}\left|\operatorname{tr}\left(\tilde{\pi}_{\lambda}(\varphi) B\right)\right|=\left(|\lambda|^{\text {Res }+n / 2}\right)\left(|\lambda|^{n / 2}\left|\operatorname{tr}\left(\tilde{\pi}_{\lambda}(\varphi) B\right)\right|\right) \text {. }
$$

Hence,

$$
\begin{aligned}
\int_{0<\lambda<\varepsilon}|\lambda|^{\operatorname{Re} s+n}\left|\operatorname{tr}\left(\tilde{\pi}_{\lambda}(\varphi) B\right)\right| d \lambda \\
<\left[\int_{0<\lambda<\varepsilon}|\lambda|^{2 \operatorname{Re} s+n} d \lambda\right]^{1 / 2} \cdot\left[\int_{0<\lambda<\varepsilon}\left|\operatorname{tr}\left(\tilde{\pi}_{\lambda}(\varphi) B\right)\right|^{2}|\lambda|^{n} d \lambda\right]^{1 / 2} .
\end{aligned}
$$

Our hypothesis on $s$ states that $2 \operatorname{Re} s+n>-1$. Hence, the integral $\left[\int_{0<\lambda<e}|\lambda|^{2 \operatorname{Res}+n} d \lambda\right]^{1 / 2}$ is finite. Call it $C^{\prime}$.

By (3.35), $\left|\operatorname{tr}\left(\tilde{\pi}_{\lambda}(\varphi) B\right)\right|^{2}<\operatorname{tr}\left(B B^{*}\right) \operatorname{tr}\left(\tilde{\pi}_{\lambda}(\varphi) \tilde{\pi}_{\lambda}(\varphi)^{*}\right)$. But by Theorem 2.7(6) and Lemma 2.13 the function $\lambda \mapsto \operatorname{tr}\left(\tilde{\pi}_{\lambda}(\varphi) \tilde{\pi}_{\lambda}(\varphi)^{*}\right)$ from $\mathbf{R}-\{0\}$ to $\mathbf{R}^{+}$is measurable. [In fact, $\tilde{\pi}_{\lambda}(\varphi) \tilde{\pi}_{\lambda}(\varphi)^{*}=\tilde{\pi}_{\lambda}\left(\varphi * \varphi^{\#}\right)$, and since $\varphi * \varphi^{\#} \in$ $C_{0}^{\infty}(G)$, one sees from (3.20) that the funaction $\lambda \mapsto \operatorname{tr}\left(\tilde{\pi}_{\lambda}(\varphi) \tilde{\pi}_{\lambda}(\varphi)^{*}\right)$ is $C^{\infty}$.] Thus,

$$
\begin{aligned}
& {\left[\int_{0<\lambda<\varepsilon}\left|\operatorname{tr}\left(\tilde{\pi}_{\lambda}(\varphi) B\right)\right|^{2}|\lambda|^{n} d \lambda\right]^{1 / 2}} \\
& \quad<\left[\operatorname{tr}\left(B B^{*}\right)\right]^{1 / 2}\left[\int_{0<\lambda<\varepsilon} \operatorname{tr}\left(\tilde{\pi}_{\lambda}(\varphi) \tilde{\pi}_{\lambda}(\varphi)^{*}\right)|\lambda|^{n} d \lambda\right]^{1 / 2} .
\end{aligned}
$$

Since $\operatorname{tr}\left(\tilde{\pi}_{\lambda}(\varphi) \tilde{\pi}_{\lambda}(\varphi)^{*}\right) \geqslant 0$ for every $\lambda \in \mathbf{R}-\{0\}$, and since the Plancherel measure $d \mu(\lambda)=|\lambda|^{n} d \lambda$, it follows from (2.28) that

$$
\begin{aligned}
{\left[\int_{0<\lambda<\varepsilon} \operatorname{tr}\left(\tilde{\pi}_{\lambda}(\varphi) \tilde{\pi}_{\lambda}(\varphi)^{*}\right)|\lambda|^{n} d \lambda\right]^{1 / 2} } \\
<\left[\int_{\mathbf{R}-\{0\}} \operatorname{tr}\left(\tilde{\pi}_{\lambda}(\varphi) \tilde{\pi}_{\lambda}(\varphi)^{*}\right)|\lambda|^{n} d \lambda\right]^{1 / 2}=\|\varphi\|_{L^{2}\left(H_{n}\right)}
\end{aligned}
$$

Letting $C=C^{\prime}\left[\operatorname{tr}\left(B B^{*}\right)\right]^{1 / 2}$ we obtain from (3.38)-(3.40) the desired result (3.37). (The first inequality in (3.37) follows automatically from the definition of $u$.) Since $L^{2}\left(H_{n}\right)$ is its own dual space it follows from (3.37) and the Hahn-Banach theorem that $u \in L^{2}\left(H_{n}\right)$. Clearly the same proof is valid for 
the domain of the integration $-\varepsilon<\lambda<0$.

LEMMA 3.6. Let $\dot{\varepsilon}>0$, and let $B$ be a trace-class operator on $L^{2}\left(R^{n}\right)$. For any $s \in \mathrm{C}$ the linear map $u: C_{0}^{\infty}\left(H_{n}\right) \rightarrow \mathrm{C}$ given by

$$
(u, \varphi)=\int_{\lambda>\varepsilon}|\lambda|^{s+n} \operatorname{tr}\left(\tilde{\pi}_{\lambda}(\varphi) B\right) d \lambda
$$

is well defined, and $u \in \Phi^{\prime}\left(H_{n}\right)$. Moreover, for any nonnegative integer $N$ such that $N>(\operatorname{Re} s+(n+1) / 2)$ there exists a constant $C$ independent of $\varphi$ such that

$$
|(u, \varphi)|<\int_{\lambda>e}|\lambda|^{\operatorname{Re} s+n}\left|\operatorname{tr}\left(\tilde{\pi}_{\lambda}(\varphi) B\right)\right| d \lambda<C\left\|Z^{N} \varphi\right\|_{L^{2}\left(H_{n}\right)} \cdot
$$

Thus, there exists $f \in L^{2}\left(H_{n}\right)$ such that $u=Z^{N}$. The same is true if we replace the domain of integration by $\lambda<-\varepsilon$.

Proof. The proof is similar to that of the preceding lemma, only we consider behavior "at $\infty$ " rather than "at 0 ". First note that $\tilde{\pi}_{\lambda}\left(Z^{\prime}\right)=$ $-\tilde{\pi}_{\lambda}(Z)=-\sqrt{-1} \lambda$. Hence by (2.13) we have

$$
\begin{aligned}
|\lambda|^{\text {Res+n}}\left|\operatorname{tr}\left(\tilde{\pi}_{\lambda}(\varphi) B\right)\right| & =\left(|\lambda|^{\text {Res+n/2-N}}\right) \cdot\left(|\lambda|^{n / 2} \cdot\left|\lambda^{N} \operatorname{tr}\left(\tilde{\pi}_{\lambda}(\varphi) B\right)\right|\right) \\
& =\left(|\lambda|^{\text {Res+n/2-N}}\right)\left(|\lambda|^{n / 2}\left|\operatorname{tr}\left(\tilde{\pi}_{\lambda}\left(Z^{N} \varphi\right) B\right)\right|\right) .
\end{aligned}
$$

Therefore

$$
\begin{aligned}
\int_{\lambda>\varepsilon}|\lambda|^{\operatorname{Re} s+n}\left|\operatorname{tr}\left(\tilde{\pi}_{\lambda}(\varphi) B\right)\right| d \lambda< & {\left[\int_{\lambda>e}|\lambda|^{2(\operatorname{Re} s+n / 2-N)} d \lambda\right]^{1 / 2} } \\
& \cdot\left[\int_{\lambda>\varepsilon}\left|\operatorname{tr}\left(\tilde{\pi}_{\lambda}\left(Z^{N} \varphi\right) B\right)\right|^{2}|\lambda|^{n} d \lambda\right]^{1 / 2} .
\end{aligned}
$$

Our hypothesis on $N$ states that $2(\operatorname{Re} s+n / 2-N)<-1$, so that

$$
\left[\int_{\lambda>\varepsilon}|\lambda|^{2(\operatorname{Re} s+n / 2-N)} d \lambda\right]^{1 / 2}<\infty .
$$

Proceeding now precisely as in the previous lemma, but with $Z^{N} \varphi$ replacing $\varphi$, we obtain (3.42). Now let $V=\left\{Z^{N} \varphi \mid \varphi \in C_{0}^{\infty}\left(H_{n}\right)\right\}$. Then $V$ is a subspace of $L^{2}\left(H_{n}\right)$, and by (3.42) the linear map $Z^{N} \varphi \mapsto(u, \varphi)$ from $V$ to $C$ is well defined and continuous. Hence, by the Hahn-Banach theorem and the fact that $L^{2}\left(H_{n}\right)$ is its own dual space, there exists $f \in L^{2}\left(H_{n}\right)$ such that, for every $\varphi \in C_{0}^{\infty}(G)$,

$$
(u, \varphi)=\left\langle Z^{N} \varphi, f\right\rangle=\left(\bar{f}, Z^{N} \varphi\right)=\left(\left(Z^{N}\right)^{t} \bar{f}, \varphi\right)=\left(Z^{N}\left((-1)^{N} \bar{f}\right), \varphi\right) .
$$

That is, $u=Z^{N}\left((-1)^{N} \bar{f}\right.$. Clearly the same proof holds for the domain of integration $\lambda<-\varepsilon$. 
We next want to show the following.

Proposition 3.7. Let $P \in \mathcal{U}\left(h_{n}\right)$ be homogeneous of degree $m$, and suppose (3.46) $\pi_{(\xi, \eta)}(P) \neq 0$ for every $(\xi, \eta) \in \mathbf{R}^{2 n}$ except $(0,0)$.

Then $P \bar{P}+Z^{2 m} \in \mathcal{Q}\left(h_{n}\right)$ is elliptic (though not homogeneous).

We first prove a lemma.

LEMMA 3.8. Let $P \in \mathcal{Q}\left(h_{n}\right)$ be homogeneous of degree $m$ and satisfy (3.46). Then $P$ is of order $m$ as a differential operator. Let $V(P) \subseteq T^{*} H_{n} / 0$ denote the real characteristic variety of $P$, i.e., the zero-set of the principal symbol $\sigma_{m}(P)$. Identifying $T_{e}^{*} H_{n}$ with $h_{n}^{*}$ we have

(3.47) $V(P)_{e}=$ the annihilator of the $2 n$-dimensional subspace of $h_{n}$ spanned by $X_{i}, Y_{i}, i=1, \ldots, n$ (with 0 deleted).

In terms of the coordinates $\xi_{i}, \eta_{i}, \tau, i=1, \ldots, n$, defined on $h_{n}^{*}$ by $X_{i}, Y_{i}, Z$ this can be expressed as

(3.48) $V(P)_{e}=\{(\xi, \eta, \tau) \mid \xi=n=0, \tau \neq 0\}$.

Proof. Let

$$
P=\sum_{|\alpha|+|\beta|+\gamma<k} a_{\alpha \beta \gamma} X_{1}^{\alpha_{1}} \cdots X_{n}^{\alpha_{n}} Y_{1}^{\beta_{1}} \cdots Y_{n}^{\beta_{n}} Z^{\gamma}, \quad a_{\alpha \beta \gamma} \in \mathbf{C} .
$$

We assume, of course, that for some $\alpha \beta \gamma$ with $|\alpha|+|\beta|+\gamma=k, a_{\alpha \beta \gamma} \neq 0$. Since $P$ is homogeneous of degree $m$,

$$
|\alpha|+|\beta|+2 \gamma=m \text { for every } \alpha \beta \gamma \text { such that } a_{\alpha \beta \gamma} \neq 0 \text {. }
$$

Since $|\alpha|+|\beta|+\gamma<k$ for every $\alpha \beta \gamma$ it follows that

$$
k<m \text {; for every } \alpha \beta \gamma \text { such that } a_{\alpha \beta \gamma} \neq 0, \gamma>m-k \text {. }
$$

But $\pi_{(\xi, \eta)}(Z)=0$ for every $(\xi, \eta)$. So, since $\pi_{(\xi, \eta)}(P) \neq 0$ for $(\xi, \eta) \neq 0$, it follows from the above expression for $P$ that there exists $\alpha \beta$ such that $a_{\alpha \beta 0} \neq 0$. Hence, by (3.50), $k=m$. That is, $P$ is of order $m$.

Thus, $\sigma_{m}(P)_{e}$, the principal symbol of $P$ at $e$, which we consider as an element of $S\left(h_{n}\right)$, the (complexified) symmetric algebra, and hence as a polynomial function on $h_{n}^{*}$, is given by

$$
\begin{aligned}
\sigma_{m}(P)_{e} & =(\sqrt{-1})^{m} \sum_{|\alpha|+|\beta|+\gamma=m} a_{\alpha \beta \gamma} X_{1}^{\alpha_{1}} \cdots X_{n}^{\alpha_{n}} Y_{1}^{\beta_{1}} \cdots Y_{n}^{\beta_{n}} Z^{\gamma} \\
& =(\sqrt{-1})^{m} \sum_{|\alpha|+|\beta|=m} a_{\alpha \beta 0} X_{1}^{\alpha_{1}} \cdots X_{n}^{\alpha_{n}} Y_{1}^{\beta_{1}} \cdots Y_{n}^{\beta_{n}} \quad(\text { by (3.49)). }
\end{aligned}
$$

Evaluating at $(\xi, \eta, \tau) \in h_{m}^{*}$ we get

$$
\begin{aligned}
\left\langle\sigma_{m}(P)_{e},(\xi, \eta, \tau)\right\rangle & =(\sqrt{-1})^{m} \sum_{|\alpha|+|\beta|=m} a_{\alpha \beta 0} \xi_{1}^{\alpha_{1}} \cdots \xi_{n}^{\alpha_{n}} \eta_{1}^{\beta_{1}} \cdots \eta_{n}^{\beta_{n}} \\
& =\pi_{(\xi, \eta)}(P) .
\end{aligned}
$$


Hence, by (3.46)

$$
\left\langle\sigma_{m}(P)_{e},(\xi, \eta, \tau)\right\rangle \neq 0 \quad \text { if }(\xi, \eta) \neq 0 .
$$

But, clearly, $\left\langle\sigma(P)_{e},(0,0, \tau)\right\rangle=0$ for all $\tau$. Hence (3.48) holds.

Since $P$ is of order $m, P \bar{P}$, and hence $P \bar{P}+Z^{2 m}$, is of order $2 m$, and so the principal symbol of $P \bar{P}+Z^{2 m}$ is given by

$$
\begin{aligned}
\left\langle\sigma_{2 m}(P \bar{P}\right. & \left.\left.+Z^{2 m}\right)_{e},(\xi, \eta, \tau)\right\rangle \\
& =\left\langle\sigma_{m}(P)_{e} \sigma_{m}(\bar{P})_{e},(\xi, \eta, \tau)\right\rangle+\left\langle\sigma_{2 m}\left(Z^{2 m}\right)_{e},(\xi, \eta, \tau)\right\rangle \\
& =(-1)^{m}\left|\left\langle\sigma_{m}(P)_{e},(\xi, \eta, \tau)\right\rangle\right|^{2}+(i \tau)^{2 m} \\
& =(-1)^{m}\left|\left\langle\sigma_{m}(P)_{e},(\xi, \eta, \tau)\right\rangle\right|^{2}+(-1)^{m}|\tau|^{2 m}
\end{aligned}
$$

Since both terms on the right-hand side of (3.54) have the same sign, the sum can be 0 only if both are 0 . Hence, by (3.48),

$$
V\left(P \bar{P}+Z^{2 m}\right)_{e}=\{(0,0, \tau) \mid \tau \neq 0\} \cap\{(\xi, \eta, 0) \mid(\xi, \eta) \neq(0,0)\}=\varnothing .
$$

Since $P \bar{P}+Z^{2 m}$ is invariant under left translation this implies that $P \bar{P}+$ $Z^{2 m}$ has empty characteristic variety, i.e., is elliptic.

As we noted at the end of $\S 2$, if $P \in \mathcal{U}\left(h_{n}\right)$ is homogeneous, so is $\bar{P}$. Also, by (3.14) and (3.15), if $P$ satisfies (3.3) so does $\bar{P}$. Thus, if we take $Q$ as in Lemma 3.3 and replace $P$ by $P Q(\bar{P} \bar{Q})=P(Q \bar{P} Q)$, it follows by Proposition 3.7 and the remarks preceding Lemma 3.3 that we can, without loss of generality, replace the hypotheses of Theorem 3.1 by the following:

(3.56) $P \in Q\left(h_{n}\right)$ is homogeneous of degree $m$.

(3.57) $\pi_{(\xi, \eta)}(P) \neq 0$ if $(\xi, \eta) \neq(0,0)$.

(3.58) $\tilde{\pi}_{1}(P), \tilde{\pi}_{-1}(P)$ have bounded right-inverses $B_{1}, B_{-1}$, respectively, which are both of trace-class.

(3.59) $P+Z^{m}$ is elliptic.

Fix $\varepsilon>0$. Then for any $s \in \mathrm{C}$ with $\operatorname{Re} s-m / 2>-(n+1) / 2$ define distributions $u_{0}^{s}, u_{\infty}^{s}, u^{s}$ by

$$
\begin{aligned}
\left(u_{0}^{s}, \varphi\right)= & (i)^{s} \int_{0<\lambda<\varepsilon}|\lambda|^{s-m / 2} \operatorname{tr}\left(\tilde{\pi}_{\lambda}(\varphi) B_{1}\right)|\lambda|^{n} d \lambda \\
& +(-i)^{s} \int_{-\varepsilon<\lambda<0}|\lambda|^{s-m / 2} \operatorname{tr}\left(\tilde{\pi}_{\lambda}(\varphi) B_{-1}\right)|\lambda|^{n} d \lambda \\
\left(u_{\infty}^{s}, \varphi\right)= & (i)^{s} \int_{\lambda>\varepsilon}|\lambda|^{s-m / 2} \operatorname{tr}\left(\tilde{\pi}_{\lambda}(\varphi) B_{1}\right)|\lambda|^{n} d \lambda \\
& +(-i)^{s} \int_{\lambda<-\varepsilon}|\lambda|^{s-m / 2} \operatorname{tr}\left(\tilde{\pi}_{\lambda}(\varphi) B_{-1}\right)|\lambda|^{n} d \lambda, \\
u^{s}= & u_{0}^{s}+u_{\infty}^{s} .
\end{aligned}
$$


[We shall always take $s$ real, in fact an integer, so there is no question of which branch of the functions $(i)^{s}$ to take.]

We know by Lemmas 3.5 and 3.6 that $u_{0}^{s}, u_{\infty}^{s}$ are distributions. In fact we know:

(3.63) For every $s, u_{0}^{s} \in L^{2}\left(H_{n}\right)$.

(3.64) For any positive integer $N>(\operatorname{Re} s-m / 2+(n+1) / 2)$ there exists $f \in L^{2}\left(H_{n}\right)$ such that $u_{\infty}^{s}=Z^{N} f$.

LEMMA 3.9. The distributions $u_{0}^{s}, u_{\infty}^{s}, u^{s}$ satisfy the following properties.

(3.65) For any nonnegative integer $k, Z^{k} u_{0}^{s}=u_{0}^{s+k}, Z^{k} u_{\infty}^{s}=u_{\infty}^{s+k}, Z^{k} u^{s}=$ $u^{s+k}$.

(3.66) For any positive integer $l>(m / 2-(n+1) / 2), P u^{l}=Z^{l} \delta$.

Proof. $\left(Z^{k} u_{0}^{s}, \varphi\right)=\left(u_{0}^{s},\left(Z^{k}\right)^{\prime} \varphi\right)$. But, by (2.13),

$$
\tilde{\pi}_{\lambda}\left(\left(Z^{k}\right)^{t} \varphi\right)=\tilde{\pi}_{\lambda}(\varphi) \tilde{\pi}_{\lambda}\left(Z^{k}\right)=(i \lambda)^{k} \tilde{\pi}_{\lambda}(\varphi) .
$$

Since $(i \lambda)^{k}=( \pm i)^{k}|\lambda|^{k}$, depending on the sign of $\lambda$, it follows that $Z^{k} u_{0}^{s}=$ $u_{0}^{s+k}$. The same argument shows that $Z^{k} u_{\infty}^{s}=u_{\infty}^{s+k}$, and, hence, that $Z^{k} u^{s}=$ $u^{s+k}$.

$$
\left(P u_{0}^{s}, \varphi\right)=\left(u_{0}^{s}, P^{\prime} \varphi\right) \text { and }\left(P u_{\infty}^{s}, \varphi\right)=\left(u_{\infty}^{s}, P^{\prime} \varphi\right)
$$

Again by (2.13),

$$
\tilde{\pi}_{\lambda}\left(P^{t} \varphi\right) v=\tilde{\pi}_{\lambda}(\varphi) \tilde{\pi}_{\lambda}(P) v \quad \text { for every } v \in \delta\left(\mathbf{R}^{n}\right) .
$$

Thus, by definition of $B_{1}, B_{-1}$ and by (3.12),

$$
\begin{gathered}
\tilde{\pi}_{\lambda}\left(P^{t} \varphi\right) B_{1} v=|\lambda|^{m / 2} \tilde{\pi}_{\lambda}(\varphi) v \quad \text { if } \lambda>0 \text { and } v \in \delta\left(\mathbf{R}^{n}\right), \\
\tilde{\pi}_{\lambda}\left(P^{t} \varphi\right) B_{-1} v=|\lambda|^{m / 2} \tilde{\pi}_{\lambda}(\varphi) v \quad \text { if } \lambda<0 \text { and } v \in \delta\left(\mathbf{R}^{n}\right) .
\end{gathered}
$$

Since both sides of (3.67), (3.68) involve bounded, in fact trace-class, operators it follows that

$$
\begin{gathered}
\tilde{\pi}_{\lambda}\left(P^{\prime} \varphi\right) B_{1}=|\lambda|^{m / 2} \tilde{\pi}_{\lambda}(\varphi) \quad \text { if } \lambda>0 \\
\tilde{\pi}_{\lambda}\left(P^{\prime} \varphi\right) B_{-1}=|\lambda|^{m / 2} \tilde{\pi}_{\lambda}(\varphi) \quad \text { if } \lambda<0 .
\end{gathered}
$$

Thus

$$
\begin{aligned}
\left(P u^{l}, \varphi\right) & =\left(P u_{0}^{l}, \varphi\right)+\left(P u_{\infty}^{l}, \varphi\right)=\int_{\mathbf{R}-\{0\}}(i \lambda)^{l} \operatorname{tr}\left(\tilde{\pi}_{\lambda}(\varphi)\right)|\lambda|^{n} d \lambda \\
& =\int_{\mathbf{R}-\{0\}} \operatorname{tr}\left(\tilde{\pi}_{\lambda}\left(\left(Z^{l}\right)^{l} \varphi\right)\right)|\lambda|^{n} d \lambda=\left(\delta,\left(Z^{l}\right)^{t} \varphi\right)=\left(Z^{\prime} \delta, \varphi\right)
\end{aligned}
$$

As an immediate corollary of (3.66) we get

Corollary 3.10. $P\left(\delta+u^{m}\right)=\left(P+Z^{m}\right) \delta$.

But by (3.59) $P+Z^{m}$ is elliptic, and so (see, for example, [13]) is locally 
solvable and hypoelliptic. In particular, there exist $v^{\prime} \in \mathscr{D}^{\prime}\left(H_{n}\right), C^{\infty}$ away from $e$, and $f^{\prime} \in C^{\infty}\left(H_{n}\right)$ such that

$$
\left(P+Z^{m}\right) v^{\prime}=\delta+f^{\prime} .
$$

Choose $\varphi \in C_{0}^{\infty}\left(H_{n}\right)$ such that $\varphi \equiv 1$ in a neighborhood of $e$. Then, letting $v=\varphi v^{\prime}$,

$$
\begin{aligned}
\left(P+Z^{m}\right) v & =\varphi\left(P+Z^{m}\right) v^{\prime}+\left[P+Z^{m}, \varphi\right] v^{\prime} \\
& =\varphi \delta+\varphi f^{\prime}+\left[P+Z^{m}, \varphi\right] v^{\prime}=\delta+f,
\end{aligned}
$$

where $f=\varphi f^{\prime}+\left[P+Z^{m}, \varphi\right] v^{\prime}$. Since $v^{\prime}$ is $C^{\infty}$ away from $e$ it follows that $f$ is $C^{\infty}$. It is also clear that $v$ and $f$ are compactly supported, and that $v$ is $C^{\infty}$ away from $e$.

Since $v \in \mathcal{E}^{\prime}\left(H_{n}\right)$, we can define a distribution $u \in \mathcal{D}^{\prime}\left(H_{n}\right)$ by

$$
u=v *\left(\delta+u^{m}\right) .
$$

Applying (3.72) and (3.73) we get

$$
\begin{aligned}
P u & =P\left(v *\left(\delta+u^{m}\right)\right)=v * P\left(\delta+u^{m}\right) \\
& =v *\left(P+Z^{m}\right) \delta=\left(P+Z^{m}\right)(v * \delta) \\
& =\left(P+Z^{m}\right)(v)=\delta+f .
\end{aligned}
$$

This proves Theorem 3.1.

4. Sufficiency for hypoellipticity. We want to prove that, at least under the stronger hypothesis that the inverses for $\tilde{\pi}_{1}(P), \tilde{\pi}_{-1}(P)$ are two-sided, the parametrix $u$ for $P$ constructed in the preceding section is $C^{\infty}$ away from $e$. To do this we need to make use of stronger boundedness properties of the inverses above than simply $L^{2}$-boundedness. Hence, the following preliminaries.

DEFINITION 4.1. For any $\delta>0$ and any nonnegative integer $k$, let $H_{(k, \delta)}\left(\mathbf{R}^{n}\right)$ be the set of all functions $v(t)$ such that $(1+|t|)^{(k-|\alpha|)} D_{t}^{\alpha} v(t) \in$ $L^{2}\left(\mathbf{R}^{n}\right)$ for all multi-indices $\alpha$ with $|\alpha| \leqslant k$. Observe that $H_{(k, \delta)}\left(\mathbf{R}^{n}\right)$ is a Hilbert-space with respect to the obvious norm, and that $\delta\left(\mathbf{R}^{n}\right) \subseteq H_{(k, \delta)}\left(\mathbf{R}^{n}\right)$. In fact, $C_{0}^{\infty}\left(\mathbf{R}^{n}\right)$, and so, a fortiori $\delta\left(\mathbf{R}^{n}\right)$, is dense in $H_{(k, \delta)}\left(\mathbf{R}^{n}\right)$. This can be seen, for example, as follows. Choose $\varphi \in C_{0}^{\infty}\left(\mathbf{R}^{n}\right)$ such that $0<\varphi<1$, and such that $\varphi \equiv 1$ in a neighborhood of 0 . Let $\varphi_{\varepsilon}(t)=\varphi(\varepsilon t)$. Then for any $v \in H_{(k, \delta)}, \varphi_{\varepsilon} v$ converges to $v$ in $H_{(k, \delta)}$ as $\varepsilon \rightarrow 0$. This shows that $H_{(k, \delta)}\left(\mathbf{R}^{n}\right) \cap$ $\mathcal{E}^{\prime}\left(\mathbf{R}^{n}\right)$ is dense in $H_{(k, \delta)}\left(\mathbf{R}^{n}\right)$. But for any $v \in H_{(k, \delta)}\left(\mathbf{R}^{n}\right) \cap \mathcal{E}^{\prime}\left(\mathbf{R}^{n}\right)$, to approximate $v$ in $H_{(k, \delta)}\left(\mathbf{R}^{n}\right)$ it suffices to approximate $v$ in $H_{k}\left(\mathbf{R}^{n}\right)$, the standard Sobolev space. Since $C_{0}^{\infty}\left(\mathbf{R}^{n}\right)$ is dense in $H_{k}\left(\mathbf{R}^{n}\right)$ we are done.

LEMMA 4.2. Let $Q \in \mathcal{Q}\left(h_{n}\right)$ be homogeneous of degree $<k$. Then $\tilde{\pi}_{1}(Q)$ and $\tilde{\pi}_{-1}(Q)$, viewed as operators from $\delta\left(\mathbf{R}^{n}\right)$ to $\delta\left(\mathbf{R}^{n}\right)$, are bounded if we give the 
domain the $H_{(k, 1)}\left(\mathbf{R}^{n}\right)$ norm, and the range the $L^{2}\left(\mathbf{R}^{n}\right)$ norm. Hence, since $\delta\left(\mathbf{R}^{n}\right)$ is dense in $H_{(k, 1)}\left(\mathbf{R}^{n}\right)$, both $\tilde{\pi}_{1}(Q)$ and $\tilde{\pi}_{-1}(Q)$ extend uniquely as bounded operators from $H_{(k, 1)}\left(\mathbf{R}^{n}\right)$ to $L^{2}\left(\mathbf{R}^{n}\right)$.

Proof. Let

$$
Q=\sum_{|\alpha|+|\beta|+\gamma<l} a_{\alpha \beta \gamma} Y_{1}^{\alpha_{1}} \cdots Y_{n}^{\alpha_{n}} X_{1}^{\beta_{1}} \cdots X_{n}^{\beta_{n}} Z^{\gamma} .
$$

We are assuming that $Q$ is homogeneous of degree $j<k$. Thus, for each nonzero coefficient $a_{\alpha \beta \gamma},|\alpha|+|\beta|+2 \gamma=j \leqslant k$. In particular, $|\alpha|+|\beta|<$ $k$. But by (3.11),

$$
\begin{aligned}
\tilde{\pi}_{1}(Q) & =\sum_{|\alpha|+|\beta|+\gamma<l} a_{\alpha \beta \gamma}(i)^{|\alpha|+\gamma} t^{\alpha} \frac{\partial^{\beta}}{\partial t}, \\
\tilde{\pi}_{-1}(Q) & =\sum_{|\alpha|+|\beta|+\gamma<l} a_{\alpha \beta \gamma}(-i)^{|\alpha|+\gamma} t^{\alpha} \frac{\partial^{\beta}}{\partial t} .
\end{aligned}
$$

Thus, it suffices to show that $t^{\alpha} \partial^{\beta} / \partial t$ defines a bounded operator from $H_{(k, 1)}\left(\mathbf{R}^{n}\right)$ to $L^{2}\left(\mathbf{R}^{n}\right)$ if $|\alpha|+|\beta| \leqslant k$. But this follows immediately from the definition of $H_{(k, 1)}$.

Notice that in the preceding proof it would have been sufficient to assume that the order of $Q$ as a differential operator $<k$. We note for future reference that the preceding proof actually shows the following stronger statement.

(4.3) If $Q \in \mathcal{U}\left(h_{n}\right)$ is homogeneous of degree $\leqslant k$, then for any integer $r \geqslant k, \tilde{\pi}_{1}(Q)$ and $\tilde{\pi}_{-1}(Q)$ define bounded operators from $H_{(r, 1)}\left(\mathbf{R}^{n}\right)$ to $H_{(r-k, 1)}\left(\mathbf{R}^{n}\right)$.

We shall need the following lemma, which will be proved in $\S 6$.

LEMmA 4.3. Let $P \in U\left(h_{n}\right)$ be homogeneous of degree $m$, and suppose that $\pi_{(\xi, \eta)}(P) \neq 0$ for every $(\xi, \eta) \in \mathbf{R}^{2 n}-\{0\}$. Fix $\lambda \in \mathbf{R}-\{0\}$. Then the following are equivalent:

(1) Neither of the equations $\tilde{\pi}_{\lambda}(P) v=0, \tilde{\pi}_{\lambda}\left(P^{*}\right) v=0$ has a nontrivial solution $v \in \mathcal{S}\left(\mathbf{R}^{n}\right)$.

(2) $\tilde{\pi}_{\lambda}(P)$ has a bounded two-sided inverse $L$, i.e., a bounded operator $L$ : $L^{2}\left(\mathbf{R}^{n}\right) \rightarrow L^{2}\left(\mathbf{R}^{n}\right)$ such that $L$ maps $\delta\left(\mathbf{R}^{n}\right)$ into $\delta\left(\mathbf{R}^{n}\right)$, and such that $\tilde{\pi}_{\lambda}(P) L=$ I on $\mathcal{S}\left(\mathbf{R}^{n}\right)$, and $L \tilde{\pi}_{\lambda}(P)=I$ on $\delta\left(\mathbf{R}^{n}\right)$. (Clearly, such an $L$, if it exists, is unique.)

If the equivalent conditions (1), (2) hold, then L-satisfies the following additional properties:

(4.4) $L$ maps $L^{2}\left(\mathbf{R}^{n}\right)$ into $H_{(m, 1)}\left(\mathbf{R}^{n}\right)$, and is bounded as an operator from $L^{2}\left(\mathbf{R}^{n}\right)$ into $H_{(m, l)}\left(\mathbf{R}^{n}\right)$.

(4.5) $\tilde{\pi}_{\lambda}(P) L=I$ on $L^{2}\left(\mathbf{R}^{n}\right)$. 
(4.6) $L \tilde{\pi}_{\lambda}(P)=I$ on $H_{(m, 1)}\left(\mathbf{R}^{n}\right)$.

(4.7) If $f \in L^{2}\left(\mathbf{R}^{n}\right)$ and $L f \in \mathcal{S}\left(\mathbf{R}^{n}\right)$, then $f \in \mathcal{S}\left(\mathbf{R}^{n}\right)$.

We can now pass to the following critical proposition.

Proposition 4.4. Let $P \in \mathcal{U}\left(h_{n}\right)$ be homogeneous of degree $m$ and satisfy

(4.8) $\pi_{(\xi, \eta)}(P) \neq 0$ for every $(\xi, \eta) \in \mathbf{R}^{2 n}-\{0\}$.

(4.9) $\tilde{\pi}_{1}(P), \tilde{\pi}_{-1}(P)$ both have bounded two-sided inverses.

Let $u^{m}$ be the distribution solution of $P u^{m}=Z^{m} \delta$ defined by (3.62). Let $\varphi$ be any function in $C_{0}^{\infty}\left(H_{n}\right)$ such that $e \notin$ support $\varphi$ (i.e., such that $\varphi \equiv 0$ in a neighborhood of $e$ ). Then

(4.10) $Z^{k}\left(\varphi u^{m}\right) \in L^{2}\left(H_{n}\right)$ for every nonnegative integer $k$.

Proof. Arguing just as in the remarks preceding (3.56) we see that if $P$ satisfies (4.8) and (4.9) then so does $\bar{P}$ (and, of course, $Q$ of Lemma 3.3), so that, without loss of generality, we can assume as before that

(4.11) $P+Z^{m}$ is elliptic.

Since $P$ satisfies (4.8) so does $P^{*}$, by (2.12). Also, by Lemma 4.3, if $P$ satisfies (4.9), then so does $P^{*}$, and hence, by the above, so does $P^{t}=\left(P^{*}\right)^{-}$. In particular,

(4.12) $\tilde{\pi}_{1}\left(P^{t}\right), \tilde{\pi}_{-1}\left(P^{t}\right)$ both have bounded two-sided inverses.

Recall that, viewing $\varphi u^{m}$ as an element in $\mathcal{E}^{\prime}\left(H_{n}\right)$, we have

(4.13) $\tilde{\pi}_{\lambda}\left(Z^{k}\left(\varphi u^{m}\right)\right)=\tilde{\pi}_{\lambda}\left(\varphi u^{m}\right) \tilde{\pi}_{\lambda}\left(\left(Z^{k}\right)^{t}\right)=(-i)^{k} \lambda^{k} \tilde{\pi}_{\lambda}\left(\varphi u^{m}\right)$.

In view of Proposition 2.10 (with $\pi_{\lambda}$ replaced by $\tilde{\pi}_{\lambda}$ ), (4.10) is equivalent to the following condition.

(4.14) For any nonnegative integer $k$ and for any $\varphi \in C_{0}^{\infty}\left(H_{n}\right)$ such that $e \notin \operatorname{supp} \varphi$, the function $\lambda \mapsto|\lambda|^{k} \tilde{\pi}_{\lambda}\left(\varphi u^{m}\right)$ lies in $L^{2}(\mathbf{R}-\{0\} ; K)$, where $K$ is the Hilbert-space of Hilbert-Schmidt operators on $L^{2}\left(\mathbf{R}^{n}\right)$, and where $\mathbf{R}$ $\{0\}$ carries the Plancherel measure $|\lambda|^{n} d \lambda$.

Our goal, then, will be to prove (4.14). First note that

$$
P\left(\varphi u^{m}\right)=\varphi P u^{m}+[P, \varphi] u^{m}=\varphi Z^{m} \delta+[P, \varphi] u^{m} .
$$

But if $e \notin \operatorname{supp} \varphi$, then $\varphi Z^{m} \delta=0$. Thus,

(4.16) For any $\varphi \in C_{0}^{\infty}\left(H_{n}\right)$ such that $e \notin \operatorname{supp} \varphi, P\left(\varphi u^{m}\right)=[P, \varphi] u^{m}$.

To reexpress $[P, \varphi]$ we need the next lemma.

LEMMA 4.5. Let $Q \in Q\left(h_{n}\right)$ be homogeneous of degree $k$, and let $\varphi \in$ $C_{0}^{\infty}\left(H_{n}\right)$. Then there is a finite set of differential operators $Q_{1}, \ldots, Q_{r} \in$ $\mathcal{Q}\left(h_{n}\right)$, each homogeneous of some degree $<k$, and $\varphi_{1}, \ldots, \varphi_{r} \in C_{0}^{\infty}\left(H_{n}\right)$ with $\operatorname{supp} \varphi_{j} \subseteq \operatorname{supp} \varphi$, so that

(4.17) $[Q, \varphi]=\sum_{j=1}^{r} Q_{j} \varphi_{j}$.

Proof. Straightforward induction on $k$.

Applying the lemma to (4.16) we get 
(4.18) For any $\varphi \in C_{0}^{\infty}\left(H_{n}\right)$ such that $e \notin$ supp $\varphi$ there exist finitely many $Q_{1}, \ldots, Q_{r} \in Q\left(h_{n}\right)$, and $\varphi_{1}, \ldots, \varphi_{r} \in C_{0}^{\infty}\left(H_{n}\right)$ such that each $Q_{j}$ is homogeneous of degree $<m$, such that $e \notin \operatorname{supp} \varphi_{j}$, and such that $P\left(\varphi u^{m}\right)=$ $\sum_{j=1}^{r} Q_{j}\left(\varphi_{j} u^{m}\right)$.

Next, we bring together some pertinent facts concerning $u_{0}^{m}$ and $u_{\infty}^{m}$. Combining (3.63) and (3.65) we get

(4.19) For any nonnegative integer $k, Z^{k} u_{0}^{m} \in L^{2}\left(H_{n}\right)$. Also, (3.64) implies, in particular,

(4.20) There exists $f \in L^{2}\left(H_{n}\right)$ and a positive integer $N$ such that $u_{\infty}^{m}=$ $Z^{N}$.

LEMMA 4.6. For any $\varphi \in C_{0}^{\infty}\left(H_{n}\right)$ the following hold.

(4.21) For any nonnegative integer $k, Z^{k}\left(\varphi u_{0}^{m}\right) \in L^{1}\left(H_{n}\right) \cap L^{2}\left(H_{n}\right)$.

(4.22) There exist $\varphi_{j} \in C_{0}^{\infty}\left(H_{n}\right), j=0, \ldots, N$, with supp $\varphi_{j} \subseteq \operatorname{supp} \varphi$ (and $\left.\varphi_{N}=\varphi\right)$ such that $\varphi u_{\infty}^{m}=\sum_{j=0}^{N} Z^{j}\left(\varphi_{j} f\right)$.

Note, in particular, that for every $j, \varphi_{j} f \in L^{1}\left(H_{n}\right) \cap L^{2}\left(H_{n}\right)$.

Proof. First we note that if $\psi \in C_{0}^{\infty}\left(H_{n}\right)$ and $g \in L^{2}\left(H_{n}\right)$, then it is obvious that $\psi g \in L^{1}\left(H_{n}\right) \cap L^{2}\left(H_{n}\right)$.

To see (4.21) observe that by an iterated application of the product rule for differentiation it follows that

$$
Z^{k}\left(\varphi u_{0}^{m}\right)=\sum_{j=0}^{k}\left(\begin{array}{l}
k \\
j
\end{array}\right)\left(Z^{j} \varphi\right)\left(Z^{k-j} u_{0}^{m}\right) .
$$

Since $Z^{j} \varphi \in C_{0}^{\infty}\left(H_{n}\right),(4.21)$ follows from (4.19) and the preceding remark.

Statement (4.22) follows from (4.20) and a variant of the proof of Lemma 4.5.

Since $\varphi u_{0}^{m} \in \mathcal{E}^{\prime}\left(H_{n}\right)$ also lies in $L^{1}\left(H_{n}\right)$, we can view $\tilde{\pi}_{\lambda}\left(\varphi u_{0}^{m}\right)$ as a bounded operator from $L^{2}\left(\mathbf{R}^{n}\right)$ to $L^{2}\left(\mathbf{R}^{n}\right)$. (See Proposition 2.6(1).) Also, by (4.22),

$$
\begin{aligned}
\tilde{\pi}_{\lambda}\left(\varphi u_{\infty}^{m}\right) & =\sum_{j=0}^{N} \tilde{\pi}_{\lambda}\left(Z^{j}\left(\varphi_{j} f\right)\right) \\
& =\sum_{j=0}^{N} \tilde{\pi}_{\lambda}\left(\varphi_{j} f\right) \tilde{\pi}_{\lambda}\left(\left(Z^{j}\right)^{t}\right)=\sum_{j=0}^{N}(-i)^{j} \lambda^{j} \tilde{\pi}_{\lambda}\left(\varphi_{j} f\right) .
\end{aligned}
$$

Since $\varphi_{j} f \in L^{1}\left(H_{n}\right)$, we again see that $\tilde{\pi}_{\lambda}\left(\varphi_{j} f\right)$, and hence, $\tilde{\pi}_{\lambda}\left(\varphi u_{\infty}^{m}\right)$, is a bounded operator from $L^{2}\left(\mathbf{R}^{n}\right)$ to $L^{2}\left(\mathbf{R}^{n}\right)$.

We are now ready to prove the main part of (4.14).

LEMMA 4.7. Let $N$ be the integer appearing in (4.20). Let $k$ be any integer $>$ $-2 N$, and let $\varphi \in C_{0}^{\infty}\left(H_{n}\right)$ such that $e \notin \operatorname{supp} \varphi$. Fix $\varepsilon>0$, and let $\mathbf{R}_{\varepsilon}=\{\lambda \in$ $\mathbf{R}|| \lambda \mid>\varepsilon\}$. Then the function $\lambda \mapsto|\lambda|^{k / 2} \tilde{\pi}_{\lambda}\left(\varphi u^{m}\right)$ lies in $L^{2}\left(\mathbf{R}_{\varepsilon} ; K\right)$, where $\mathbf{R}_{\varepsilon}$ 
is provided with the Plancherel measure $|\lambda|^{n} d \lambda$.

Proof. The proof will proceed by induction on $k$. First we take $k=-2 N$. Thus, we want to show:

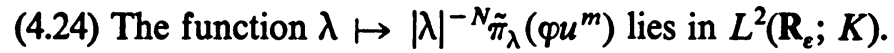
Since

(4.25) $\tilde{\pi}_{\lambda}\left(\varphi u^{m}\right)=\tilde{\pi}_{\lambda}\left(\varphi u_{0}^{m}\right)+\tilde{\pi}_{\lambda}\left(\varphi u_{\infty}^{m}\right)$ it suffices to show

(4.24a) $\lambda \mapsto|\lambda|^{-N} \tilde{\pi}_{\lambda}\left(\varphi u_{0}^{m}\right)$ lies in $L^{2}\left(\mathbf{R}_{\varepsilon} ; K\right)$.

(4.24b) $\lambda \mapsto|\lambda|^{-N} \tilde{\pi}_{\lambda}\left(\varphi u_{\infty}^{m}\right)$ lies in $L^{2}\left(\mathbf{R}_{\varepsilon} ; K\right)$.

By (4.21) we know, in particular, that $\varphi u_{0}^{m} \in L^{1}\left(H_{n}\right) \cap L^{2}\left(H_{n}\right)$. Thus, by the Plancherel theorem, $\lambda \mapsto \tilde{\pi}_{\lambda}\left(\varphi u_{0}^{m}\right)$ lies in $L^{2}(\mathbf{R}-\{0\} ; K)$, and so, by restriction, it also lies in $L^{2}\left(\mathbf{R}_{e} ; K\right)$. Since $\lambda \mapsto|\lambda|^{-N}$ is $C^{\infty}$ and bounded on $\mathbf{R}_{\varepsilon}$ (in particular, measurable and bounded), (4.24a) follows.

To prove (4.24b) we use (4.23) and argue as above. Since $\varphi_{j} f \in L^{1}\left(H_{n}\right) \cap$ $L^{2}\left(H_{n}\right)$, the Plancherel theorem implies that $\lambda \mapsto \tilde{\pi}_{\lambda}\left(\varphi_{j} f\right)$ lies in $L^{2}(\mathbf{R}-$ $\{0\} ; K)$, and, hence, in $L^{2}\left(\mathbf{R}_{\varepsilon} ; K\right)$. For any $0 \leqslant j \leqslant N$ the function $\lambda \mapsto$ $|\lambda|^{-N} \lambda^{j}$ is $C^{\infty}$ and bounded on $\mathbf{R}_{\varepsilon}$. Hence (4.24b) follows from (4.23). Thus, we have proved the lemma for $k=-2 N$. Notice that, thus far, we have not used the fact that $e \notin \operatorname{supp} \varphi$. This assumption will be needed when we use (4.18) in the proof of the inductive step.

We now assume that we have shown

(4.26) For any integer $k$ such that $-2 N \leqslant k \leqslant l$, and for any $\varphi \in C_{0}^{\infty}\left(H_{n}\right)$ such that $e \notin \operatorname{supp} \varphi$, the function $\lambda \mapsto|\lambda|^{k / 2} \tilde{\pi}_{\lambda}\left(\varphi u^{m}\right)$ lies in $L^{2}\left(\mathbf{R}_{e} ; K\right)$.

Since for any nonnegative integer $r$ the function $\lambda \mapsto|\lambda|^{-r / 2}$ is $C^{\infty}$ and bounded on $\mathbf{R}_{e}$, it follows that the function $\lambda \mapsto|\lambda|^{(k-r) / 2} \tilde{\pi}_{\lambda}\left(\varphi u^{m}\right)$ also lies in $L^{2}\left(R_{e} ; K\right)$. That is, (4.26) is equivalent to

(4.27) For any integer $k<l$ and for any $\varphi \in C_{0}^{\infty}\left(H_{n}\right)$ such that $e \notin$ supp $\varphi$, the function $\lambda \mapsto|\lambda|^{k / 2} \tilde{\pi}_{\lambda}\left(\varphi u^{m}\right)$ lies in $L^{2}\left(\mathbf{R}_{e} ; K\right)$.

We shall show that (4.27) implies:

(4.28) For any $\varphi \in C_{0}^{\infty}\left(H_{n}\right)$ such that $e \notin \operatorname{supp} \varphi$, the function $\lambda \mapsto$ $|\lambda|^{(l+1) / 2} \tilde{\pi}_{\lambda}\left(\varphi u^{m}\right)$ lies in $L^{2}\left(\mathbf{R}_{e} ; K\right)$.

Let $\varphi \in C_{0}^{\infty}\left(H_{n}\right)$ such that $e \notin \operatorname{supp} \varphi$. Applying $\tilde{\pi}_{\lambda}$ to the compactly supported distributions in (4.18) we get

$$
\tilde{\pi}_{\lambda}\left(P\left(\varphi u^{m}\right)\right)=\sum_{j=1}^{r} \tilde{\pi}_{\lambda}\left(Q_{j}\left(\varphi_{j} u^{m}\right)\right) .
$$

So, by Proposition 2.6 (6),

$$
\tilde{\pi}_{\lambda}\left(\varphi u^{m}\right) \tilde{\pi}_{\lambda}\left(P^{t}\right)=\sum_{j=1}^{r} \tilde{\pi}_{\lambda}\left(\varphi_{j} u^{m}\right) \tilde{\pi}_{\lambda}\left(Q_{j}^{t}\right) .
$$

Here both sides may be viewed as unbounded operators on $\delta\left(\mathbf{R}^{n}\right)$. Recall, 
however (see remarks preceding Lemma 4.7), that $\tilde{\pi}_{\lambda}\left(\varphi u_{0}^{m}\right)$ and $\tilde{\pi}_{\lambda}\left(\varphi u_{\infty}^{m}\right)$, and hence, by (4.25), $\tilde{\pi}_{\lambda}\left(\varphi u^{m}\right)$ are bounded operators from $L^{2}\left(\mathbf{R}^{n}\right)$ to $L^{2}\left(\mathbf{R}^{n}\right)$.

Each $Q_{j}$ is homogeneous of degree $s_{j}<m$, so the same is true of $Q_{j}^{t}$. Applying (3.12) we get

$$
\begin{aligned}
& |\lambda|^{m / 2} \tilde{\pi}_{\lambda}\left(\varphi u^{m}\right) \tilde{\pi}_{1}\left(P^{t}\right)=\sum_{j=1}^{r}|\lambda|^{s / 2} \tilde{\pi}_{\lambda}\left(\varphi_{j} u^{m}\right) \tilde{\pi}_{1}\left(Q_{j}^{t}\right) \text { for } \lambda>0, \\
& |\lambda|^{m / 2} \tilde{\pi}_{\lambda}\left(\varphi u^{m}\right) \tilde{\pi}_{-1}\left(P^{t}\right)=\sum_{j=1}^{r}|\lambda|^{s / 2} / \tilde{\pi}_{\lambda}\left(\varphi_{j} u^{m}\right) \tilde{\pi}_{-1}\left(Q_{j}^{t}\right) \text { for } \lambda<0 .
\end{aligned}
$$

By (4.12), $\tilde{\pi}_{1}\left(P^{t}\right)$ and $\tilde{\pi}_{-1}\left(P^{t}\right)$ have bounded two-sided inverses, $L_{1}, L_{-1}$, respectively. Since $P^{t}$ is homogeneous of degree $m$ and satisfies (4.8), it follows from Lemma 4.3 that

(4.32) $L_{1}, L_{-1}$ map $L^{2}\left(\mathbf{R}^{n}\right)$ into $H_{(m, 1)}\left(\mathbf{R}^{n}\right)$, and are bounded as operators from $L^{2}\left(\mathbf{R}^{n}\right)$ into $H_{(m, 1)}\left(\mathbf{R}^{n}\right)$.

Since $s_{j}<m$ it follows from Lemma 4.2 that

(4.33) For every $j=1, \ldots, r, \tilde{\pi}_{1}\left(Q_{j}^{t}\right), \tilde{\pi}_{-1}\left(Q_{j}^{t}\right)$ are bounded operators from $H_{(m, 1)}\left(\mathbf{R}^{n}\right)$ to $L^{2}\left(\mathbf{R}^{n}\right)$. (Of course, (4.33) would hold even if $s_{j}$ were equal to $m$.)

Letting $T_{1, j}=\tilde{\pi}_{1}\left(Q_{j}^{\prime}\right) L_{1}, T_{-1, j}=\tilde{\pi}_{-1}\left(Q_{j}^{t}\right) L_{-1}$, we know by (4.32) and (4.33) that

(4.34) $T_{1, j}, T_{-1, j}$ are bounded operators from $L^{2}\left(\mathbf{R}^{n}\right)$ to $L^{2}\left(\mathbf{R}^{n}\right)$ which map $\delta\left(\mathbf{R}^{n}\right)$ into $\delta\left(\mathbf{R}^{n}\right)$.

Applying $L_{1}, L_{-1}$ to (4.31a), (4.31b), respectively, we get

$$
\begin{aligned}
& \tilde{\pi}_{\lambda}\left(\varphi u^{m}\right)=\sum_{j=1}^{r}|\lambda|^{\left(s_{j}-m\right) / 2} \tilde{\pi}_{\lambda}\left(\varphi_{j} u^{m}\right) T_{1, j} \text { for } \lambda>0, \\
& \tilde{\pi}_{\lambda}\left(\varphi u^{m}\right)=\sum_{j=1}^{r}|\lambda|^{\left(s_{j}-m\right) / 2} \tilde{\pi}_{\lambda}\left(\varphi_{j} u^{m}\right) T_{-1, j} \text { for } \lambda<0 .
\end{aligned}
$$

Initially we know that $(4.35 \mathrm{a})$ and $(4.35 \mathrm{~b})$ are valid as equations between operators defined on $\delta\left(\mathbf{R}^{n}\right)$. However, since $\tilde{\pi}_{\lambda}\left(\varphi u^{m}\right), \tilde{\pi}_{\lambda}\left(\varphi_{j} u^{m}\right), T_{1, j}, T_{-1, j}$ all are bounded operators from $L^{2}\left(\mathbf{R}^{n}\right)$ to $L^{2}\left(\mathbf{R}^{n}\right)$, and since $\delta\left(\mathbf{R}^{n}\right)$ is dense in $L^{2}\left(R^{n}\right)$, we can view (4.35a) and (4.35b) as equations between bounded operators on $L^{2}\left(\mathbf{R}^{n}\right)$.

Multiplying (4.35a), (4.35b) by $|\lambda|^{(l+1) / 2}$ we get

$$
\begin{aligned}
& |\lambda|^{(l+1) / 2} \tilde{\pi}_{\lambda}\left(\varphi u^{m}\right)=\sum_{j=1}^{r}|\lambda|^{\left(l+1+s_{j}-m\right) / 2} \tilde{\pi}_{\lambda}\left(\varphi_{j} u^{m}\right) T_{1_{j}} \text { for } \lambda>0, \\
& |\lambda|^{(l+1) / 2} \tilde{\pi}_{\lambda}\left(\varphi u^{m}\right)=\sum_{j=1}^{r}|\lambda|^{\left(l+1+s_{j}-m\right) / 2} \tilde{\pi}_{\lambda}\left(\varphi_{j} u^{m}\right) T_{-1, j} \text { for } \lambda<0 .
\end{aligned}
$$

Since $s_{j}-m<0, l+1+s_{j}-m \leqslant l$. Hence, by the inductive hypothesis (4.27) the function $\lambda \mapsto|\lambda|^{\left(l+1+s_{j}-m\right) / 2} \tilde{\pi}_{\lambda}\left(\varphi_{j} u^{m}\right)$ lies in $L^{2}\left(\mathbf{R}_{e} ; K\right)$, and so, by 
restriction, in $L^{2}\left(\mathbf{R}_{\varepsilon}^{+} ; K\right), L^{2}\left(\mathbf{R}_{\varepsilon}^{-} ; K\right)$, where $\mathbf{R}_{\varepsilon}^{+}=\{\lambda \in \mathbf{R} \mid \lambda>\varepsilon\}$ and $\mathbf{R}_{\varepsilon}^{-}=$ $\{\lambda \in \mathbf{R} \mid \lambda<-\varepsilon\}$.

But if $(\Omega, \mu)$ is any measure space, $H$ a Hilbert-space, and $T$ a bounded linear operator from $H$ to $H$, then for any function $\lambda \mapsto f(\lambda)$ in $L^{2}(\Omega ; H)$, the function $\lambda \mapsto T(f(\lambda))$ also lies in $L^{2}(\Omega ; H)$. Indeed, it is easy to see that $\lambda \mapsto T(f(\lambda))$ is $\mu$-measurable, and

$$
\left[\int_{\Omega}\|T(f(\lambda))\|_{H}^{2} d \mu(\lambda)\right]^{1 / 2} \leqslant\|T\|\left[\int_{\Omega}\|f(\lambda)\|_{H}^{2} d \mu(\lambda)\right]^{1 / 2} .
$$

Returning to our particular case, any bounded linear operator $T: L^{2}\left(\mathbf{R}^{n}\right) \rightarrow$ $L^{2}\left(\mathbf{R}^{n}\right)$ determines a bounded linear operator: $K \rightarrow K, K$ being the Hilbertspace of Hilbert-Schmidt operators on $L^{2}\left(R^{n}\right)$, defined by $S \in K \mapsto S T \in$ $K$.

Thus, the function

$$
\lambda \mapsto|\lambda|^{\left(l+1+s_{j}-m\right) / 2} \tilde{\pi}_{\lambda}\left(\varphi_{j} u^{m}\right) T_{1, j}
$$

lies in $L^{2}\left(\mathbf{R}_{e}^{+} ; K\right)$, and the function

$$
\lambda \mapsto|\lambda|^{\left(l+1+s_{j}-m\right) / 2} \tilde{\pi}_{\lambda}\left(\varphi_{j} u^{m}\right) T_{-1, j}
$$

lies in $L^{2}\left(\mathrm{R}_{e}^{-} ; K\right)$. Hence, by (4.36a), (4.36b), the function

$$
\lambda \mapsto|\lambda|^{(l+1) / 2} \tilde{\pi}_{\lambda}\left(\varphi u^{m}\right)
$$

lies in $L^{2}\left(\mathbf{R}_{\varepsilon} ; K\right)$. This proves (4.28).

To conclude the proof of (4.14) it suffices to show

LEMMA 4.8. Let $k$ be any nonnegative integer, and let $\varphi \in C_{0}^{\infty}\left(H_{n}\right)$ such that $e \notin \operatorname{supp} \varphi$. Then the function $\lambda \mapsto|\lambda|^{k} \tilde{\pi}_{\lambda}\left(\varphi u^{m}\right)$ lies in $L^{2}(\{0<|\lambda|<\varepsilon\} ; K)$.

Proof. As a matter of fact, we do not require the hypothesis that $e \notin$ supp $\varphi$.

By (4.25) it suffices to show that each of the functions $\lambda \mapsto|\lambda|^{k} \tilde{\pi}_{\lambda}\left(\varphi u_{0}^{m}\right)$, $\lambda \mapsto|\lambda|^{k} \tilde{\pi}_{\lambda}\left(\varphi u_{\infty}^{m}\right)$ lies in $L^{2}(\{0<|\lambda| \leqslant \varepsilon\} ; K)$. For the first of these functions this follows from (4.21). On the other hand, it follows from (4.23) that

$$
|\lambda|^{k} \tilde{\pi}_{\lambda}\left(\varphi u_{\infty}^{m}\right)=\sum_{j=0}^{N}(-i)^{j}|\lambda|^{k} \lambda^{j} \tilde{\pi}_{\lambda}\left(\varphi_{j} f\right)
$$

where $\varphi_{j} f \in L^{1}\left(H_{n}\right) \cap L^{2}\left(H_{n}\right)$, so that $\lambda \mapsto \tilde{\pi}_{\lambda}\left(\varphi_{j} f\right)$ lies in $L^{2}(\{0<|\lambda|<$ $\varepsilon\} ; K)$. Since $\lambda \mapsto|\lambda|^{k} \lambda^{j}$ is bounded and $C^{\infty}$ on $\{0<|\lambda|<\varepsilon\}$, it follows that $\lambda \mapsto|\lambda|^{k} \tilde{\pi}_{\lambda}\left(\varphi u_{\infty}^{m}\right)$ lies in $L^{2}(\{0 \lambda|\lambda| \leqslant \varepsilon\} ; K)$.

Thus we have proved (4.14), and, therefore, Proposition 4.4.

We can now prove the main result of this section.

TheOREM 4.9. Let $P \in \mathcal{U}\left(h_{n}\right)$ be homogeneous of degree $m$ and satisfy (1) $\pi_{(\xi, \eta)}(P) \neq 0$ for every $(\xi, \eta) \in \mathbf{R}^{2 n}-\{0\}$, 
(2) $\tilde{\pi}_{1}(P), \tilde{\pi}_{-1}(P)$ both have bounded two-sided inverses.

Then the parametrix $u=v *\left(\delta+u^{m}\right)$ for $P$ constructed in $\S 3$ is $C^{\infty}$ away from e.

Proof. As we noted in (4.11), we can assume without loss of generality that $P+Z^{m}$ is elliptic, a fact that we needed to obtain $v$.

First we show that $u^{m}$ is $C^{\infty}$ away from $e$. Since $Z$ lies in the center of $\mathcal{Q}\left(h_{n}\right)$, so does $Z^{m}$. In particular, $Z^{m}$ commutes with $P$. Hence, for every positive integer $k$,

$$
\left(P+Z^{m}\right)^{k} u^{m}=\sum_{j=0}^{k}\left(\begin{array}{l}
k \\
j
\end{array}\right) Z^{m j} P^{k-j} u^{m} .
$$

Hence, since $P u^{m}=Z^{m} \delta$, which is supported at $e$,

(4.39) $Z^{m j} P^{k-j} u^{m}$ is supported at $e$ when $j<k$.

Thus

(4.40) $\varphi\left(P+Z^{m}\right)^{k} u^{m}=\varphi Z^{m k} u^{m}$ for any $\varphi \in C_{0}^{\infty}\left(H_{n}\right)$ such that $e \notin$ supp $\varphi$.

By the same argument used to prove (4.22) we see that

(4.41) $\varphi Z^{m k} u^{m}=\sum_{j=0}^{m k} Z^{j}\left(\varphi_{j} u^{m}\right)$, where $\varphi_{j} \in C_{0}^{\infty}\left(H_{n}\right)$ such that supp $\varphi_{j} \subseteq$ supp $\varphi$.

In particular, $e \notin \operatorname{supp} \varphi_{j}$. Applying Proposition 4.4, we see that $Z^{j}\left(\varphi_{j} u^{m}\right)$ $\in L^{2}\left(H_{n}\right)$ for every $j=0, \ldots, m k$. Hence, $\varphi Z^{m k} u^{m} \in L^{2}\left(H_{n}\right)$, and so, by (4.40),

(4.42) $\varphi\left(P+Z^{m}\right)^{k} u^{m} \in L^{2}\left(H_{n}\right)$ for every positive integer $k$ and for every $\varphi \in C_{0}^{\infty}\left(H_{n}\right)$ such that $e \notin \operatorname{supp} \varphi$. Since $\left(P+Z^{m}\right)^{k}$ is an elliptic operator of order $m k$, it follows by the standard regularity results for elliptic operators that $u^{m} \in H_{\mathrm{loc}}^{m k}\left(H_{n}-\{e\}\right)$, where $H_{\mathrm{loc}}^{s}$ denotes the usual Sobolev space. Since $k$ is arbitrary it follows by the Sobolev lemma that $u^{m} \in C^{\infty}\left(H_{n}\right.$ $\{e\})$. That is, $u^{m}$ is $C^{\infty}$ away from $e$.

Hence, $\delta+u^{m}$ is $C^{\infty}$ away from $e$. But, by construction, $v \in \mathcal{E}^{\prime}\left(H_{n}\right)$ is $C^{\infty}$ away from $e$. Since convolution by a distribution which is $C^{\infty}$ away from $e$ does not increase singular support, it follows that $v *\left(\delta+u^{m}\right)$ is $C^{\infty}$ away frome.

REMARKS. 1. Since $u$ is $C^{\infty}$ away from $e$ we can, by multiplying by a suitable cutoff function, obtain a compactly-supported parametrix for $P, C^{\infty}$ away from $e$. (See the remarks following (3.7).)

2. As we observed at the beginning of the proof of Proposition 4.4, if $P$ satisfies (1) and (2) of Theorem 4.9 then so do $P^{*}, \bar{P}, P^{t}$.

It is a well-known result that if $P$ has a parametrix which is $C^{\infty}$ away from $e$, then $P^{\prime}$ is hypoelliptic. (See, for example, Trèves [24, Chapter 52].) Hence, by Theorem 4.9 and Remark 2 above, condition (1.2) in Theorem 1.2 implies condition (1.1). 
5. Necessity for hypoellipticity. In this section we prove

THEOREM 5.1. Let $P \in \mathcal{U}\left(h_{n}\right)$ be homogeneous, say of degree $m$, and suppose that both $P$ and $P^{t}$ are hypoelliptic. Then:

$(5.1) \pi_{(\xi, \eta)}(P) \neq 0$ for every $(\xi, \eta) \in \mathbf{R}^{2 n}-\{0\}$.

(5.2) Neither of the equations $\tilde{\pi}_{\lambda}(P) v=0, \tilde{\pi}_{\lambda}\left(P^{*}\right) v=0$ has a nonzero solution $v \in \mathcal{S}\left(\mathbf{R}^{n}\right)$, for any $\lambda \in \mathbf{R}-\{0\}$.

REMARK. Since a distribution $u$ is $C^{\infty}$ on an open set if and only if the same is true for $\bar{u}$, it follows that $P$ is hypoelliptic if and only if $\bar{P}$ is hypoelliptic. Since $P^{*}=\left(P^{t}\right)^{-}$, this implies also that $P^{t}$ is hypoelliptic if and only if $P^{*}$ is hypoelliptic. (Of course, the same holds with "hypoelliptic" replaced by "locally solvable".)

The next lemma is crucial, since it provides us with compactly supported distributions, to which we can then apply unitary representations.

LEMma 5.2. Let $P$ be a differential operator on $H_{n}$ such that both $P$ and $P^{t}$ are hypoelliptic. Then there exist distributions $u_{1}, u_{2} \in \mathcal{E}^{\prime}\left(H_{n}\right)$, both $C^{\infty}$ away from $e$, and functions $f_{1}, f_{2} \in C_{0}^{\infty}\left(H_{n}\right)$ such that

(5.3a) $P u_{1}=\delta+f_{1}$,

(5.3b) $P^{t} u_{2}=\delta+f_{2}$

Proof. This follows immediately from Theorem 52.2 in Trèves [24], and from the use of cutoff functions as the proof of (3.73).

As motivation for the proof of Theorem 5.1, we shall first present a treatment of the simpler case $G=\mathbf{R}^{n}$, with addition as the group operation, and with the usual dilations $x \mapsto r x$. The result is, of course, well known, being a special case of Hörmander's characterization [13, Chapter IV] of hypoellipticity for, not necessarily homogeneous, constant-coefficient differential operators. However, the homogeneous case allows a particularly simple treatment. We should also point out a special feature of the case $G=\mathbf{R}^{n}$ which follows from the fact that $G$ is commutative. If

$$
P=\sum_{|\alpha|<m} a_{\alpha} \frac{\partial^{\alpha_{1}}}{\partial x_{1}} \cdots \frac{\partial^{\alpha_{n}}}{\partial x_{n}}, \quad a_{\alpha} \in \mathbf{C}
$$

then

$$
P^{\prime}=\sum_{|\alpha|<m} a_{\alpha}\left(-\frac{\partial}{\partial x_{1}}\right)^{\alpha_{1}} \cdots\left(-\frac{\partial}{\partial x_{n}}\right)^{\alpha_{n}},
$$

and so for any $u \in \mathscr{Q}^{\prime}\left(\mathbf{R}^{n}\right), P^{\prime}(u(-x))=(P u)(-x)$. Hence a constant-coefficient differential operator $P$ is hypoelliptic if and only if $P^{t}$ is hypoelliptic.

Proposition 5.3. Let $P$ be a left-invariant (hence bi-invariant) differential 
operator on $\mathbf{R}^{n}$, i.e., a constant-coefficient differential operator. Suppose, moreover, that $P$ is homogeneous with respect to dilations, which means precisely that $P$ has only terms of highest order in the usual sense. Then the following are equivalent.

(5.4) $\pi(P)$ is invertible for every unitary irreducible representation $\pi$ of $\mathbf{R}^{n}$ except the trivial, identity representation. (This just says that $P$ is elliptic.)

(5.5) $P$ is hypoelliptic.

(5.6) There exists $u \in \mathcal{E}^{\prime}\left(\mathbf{R}^{n}\right)$ such that $P u=\delta+f$ for some $f \in C_{0}^{\infty}\left(\mathbf{R}^{n}\right)$.

Proof. First recall that the irreducible unitary representations of $\mathbf{R}^{n}$ are all 1-dimensional and are parametrized by $\mathbf{R}^{n}$ as follows.

$$
\pi_{\xi}(x)=e^{-i x \cdot \xi}, \quad \xi \in \mathbf{R}^{n} .
$$

We mean, of course, that $\pi_{\xi}(x)$ acts on the 1-dimensional Hilbert space $C$ by multiplication by $e^{-i x \xi}$. The identity representation corresponds to $\xi=0$.

The left-invariant vector-fields $\partial / \partial x_{1}, \ldots, \partial / \partial x_{n}$ form a basis for the Lie algebra of $\mathbf{R}^{n}$. Letting $\delta_{j}$ be the vector in $\mathbf{R}^{n}$ with $j$ th entry equal to 1 and all other entries 0 , we see

$$
\begin{aligned}
\pi_{\xi}\left(\frac{\partial}{\partial x_{j}}\right) & =\left.\frac{d}{d t}\right|_{t=0} \pi_{\xi}\left(\exp t \frac{\partial}{\partial x_{j}}\right)=\left.\frac{d}{d t}\right|_{t=0} \pi_{\xi}\left(t \delta_{j}\right) \\
& =\left.\frac{d}{d t}\right|_{t=0} e^{-i t \xi}=-i \xi_{j} .
\end{aligned}
$$

Hence, $\pi_{\xi}\left(D_{x_{j}}\right)=-\xi_{j}$, where $D_{x_{j}}=(1 / i) \partial / \partial x_{j}$.

Taking the appropriate multiple of Lebesgue measure as Haar measure we see from (5.7) that

$$
\pi_{\xi}(u)=\hat{u}(\xi) \text { for any } u \in \mathcal{E}^{\prime}\left(\mathbf{R}^{n}\right),
$$

where $\hat{u}$ denotes the Fourier transform of $u$.

Since $P$ is constant-coefficient and homogeneous, say of degree $m$, we can write $P$ as

$$
P=\sum_{|\alpha|=m} a_{\alpha} D_{x_{1}}^{\alpha_{1}} \cdots D_{x_{n}}^{\alpha_{n}}, \quad a_{\alpha} \in \mathbf{C},
$$

so that $\sigma(P)$, the usual principal symbol of $P$, is given by

$$
\sigma(P)(\xi)=\sum_{|\alpha|=m} a_{\alpha} \xi_{1}^{\alpha_{1}} \cdots \xi_{n}^{\alpha_{n}}=(-1)^{m} \pi_{\xi}(P) .
$$

This shows that (5.4) states precisely that $P$ is elliptic, and hence implies (5.5).

Every constant-coefficient differential operator on $\mathbf{R}^{n}$ has a fundamental solution, i.e., there exists $v \in \mathscr{D}^{\prime}\left(\mathrm{R}^{n}\right)$ such that $P v=\delta$. If $P$ is hypoelliptic then $v$ is $C^{\infty}$ away from 0 , and so $P u=\delta+f$, where $f \in C_{0}^{\infty}\left(\mathbf{R}^{n}\right)$, if we take $u=\varphi v$ with $\varphi \in C_{0}^{\infty}\left(\mathbf{R}^{n}\right)$ such that $\varphi \equiv 1$ in a neighborhood of 0 . Hence (5.5) implies (5.6). 
Finally (and this is what we are mainly interested in showing) we prove that (5.6) implies (5.4). Since $u$ (and therefore $P u$ ) is compactly supported we can apply unitary representations. Thus it follows from (5.6), (5.9) that

$$
\hat{u}(\xi) \pi_{\xi}\left(P^{t}\right)=\pi_{\xi}(P u)=\pi_{\xi}(\delta)+\pi_{\xi}(f)=1+\hat{f}(\xi) .
$$

But from (5.10) it follows that $P^{t}=(-1)^{m} P$. Hence, by (5.11) and (5.12),

$$
\hat{u}(\xi) \sigma(P)(\xi)=1+\hat{f}(\xi) \text {. }
$$

Replacing $\xi$ by $r \xi, r \in \mathbf{R}^{+}$, and noting that

$$
\sigma(P)(r \xi)=r^{m} \sigma(P)(\xi)
$$

we get

$$
r^{m} \hat{u}(r \xi) \cdot \sigma(P)(\xi)=1+\hat{f}(r \xi) \text { for every } r \in \mathbf{R}^{+} .
$$

Since $f \in C_{0}^{\infty}\left(\mathbf{R}^{n}\right), \hat{f} \in \delta\left(\mathbf{R}^{n}\right)$. In particular, for fixed $\xi \neq 0,|\hat{f}(r \xi)|<\varepsilon$ for $r$ sufficiently large. Therefore, for $r$ sufficiently large the right-hand side of (5.15) does not equal 0 , and so, by $(5.15), \sigma(P)(\xi) \neq 0$. That is, $P$ is elliptic.

We next pass to the proof of Theorem 5.1. Applying Lemma 5.2 (but with $P$ replaced by $\bar{P}$ in (5.3a)) we obtain

(5.16a) $\pi_{(\xi, \eta)}\left(u_{1}\right) \overline{\pi_{(\xi, \eta)}(P)}=1+\pi_{(\xi, \eta)}\left(f_{1}\right)$ for every $(\xi, \eta) \in \mathbf{R}^{2 n}-\{0\}$,

(5.16b) $\pi_{(\xi, \eta)}\left(u_{2}\right) \pi_{(\xi, \eta)}(P)=1+\pi_{(\xi, \eta)}\left(f_{2}\right)$ for every $(\xi, \eta) \in \mathbf{R}^{2 n}-\{0\}$,

$$
\begin{aligned}
& \tilde{\pi}_{\lambda}\left(u_{1}\right) \tilde{\pi}_{\lambda}(P)^{*} v=\left(I+\tilde{\pi}_{\lambda}\left(f_{1}\right)\right) v \\
& \quad \text { for every } \lambda \in \mathbf{R}-\{0\} \text { and } v \in \delta\left(\mathbf{R}^{n}\right), \\
& \tilde{\pi}_{\lambda}\left(u_{2}\right) \tilde{\pi}_{\lambda}(P) v=\left(I+\tilde{\pi}_{\lambda}\left(f_{2}\right)\right) v \\
& \quad \text { for every } \lambda \in \mathbf{R}-\{0\} \text { and } v \in \delta\left(\mathbf{R}^{n}\right) .
\end{aligned}
$$

It follows from (3.7) that if we use exponential coordinates on $H_{n}$,

$$
\pi_{(\xi, \eta)}(\varphi)=\hat{\varphi}(-\xi,-\eta, 0) \text { for any } \varphi \in C_{0}^{\infty}\left(H_{n}\right) .
$$

Here $\hat{\varphi}$ denotes the Fourier transform of $\varphi$.

Applying this, together with (3.12) and (3.13), to the above we see that for any $r \in \mathbf{R}^{+}$,

$$
\begin{gathered}
r^{m} \pi_{(r \xi, \eta)}\left(u_{1}\right) \overline{\pi_{(\xi, \eta)}(P)}=1+\hat{f}_{1}(-r \xi,-r, 0), \\
r^{m_{\left.\pi_{(r \xi, \eta)}\right)}}\left(u_{2}\right) \pi_{(\xi, \eta)}(P)=1+\hat{f}_{2}(-r \xi,-m, 0), \\
r^{m / 2} \tilde{\pi}_{r \lambda}\left(u_{1}\right) \tilde{\pi}_{\lambda}(P)^{*} v=\left(I+\tilde{\pi}_{r \lambda}\left(f_{1}\right)\right) v, \\
r^{m / 2} \tilde{\pi}_{r \lambda}\left(u_{2}\right) \tilde{\pi}_{\lambda}(P) v=\left(I+\tilde{\pi}_{r \lambda}\left(f_{2}\right)\right) v .
\end{gathered}
$$

Using either (5.19a) or (5.19b) and arguing as in the preceding proposition, we obtain (5.1). But, by Theorem 2.7(5), with $\tilde{\pi}_{\lambda}$ replacing $\pi_{\lambda},\left\|\tilde{\pi}_{r \lambda}\left(f_{1}\right)\right\|<\varepsilon$ 
and $\left\|\tilde{\pi}_{r \lambda}\left(f_{2}\right)\right\|<\varepsilon$ for $r$ sufficiently large, and fixed $\lambda$. Hence, for $r$ sufficiently large, $I+\tilde{\pi}_{r \lambda}\left(f_{1}\right)$ and $I+\tilde{\pi}_{r \lambda}\left(f_{2}\right)$ are invertible bounded linear operators on $L^{2}\left(\mathbf{R}^{n}\right)$. In particular, the right-hand sides of $(5.20 \mathrm{a}),(5.20 \mathrm{~b})$ cannot be 0 unless $v=0$. Thus (5.2) follows from (5.20a) and (5.20b).

Theorem 5.1 shows that $(1.1) \Rightarrow(1.3)$. Also, we saw at the end of $\$ 4$ that $(1.2) \Rightarrow(1.1)$. But, by Lemma $4.3,(1.3) \Rightarrow(1.1)$. Hence, modulo Lemma 4.3, which will be proved in $\$ 6$, we have proved Theorem 1.2.

6. Grušin operators. Grušin [9] (see also [8], [10]) derives a characterization of hypoellipticity for a class of differential operators arising in the study of degenerate-elliptic boundary-value problems. In the process he needs to derive certain results concerning the partial Fourier transforms of these operators. By introducing an "equivalence" between certain of the Grušin operators and operators on $H_{n}$ we are able to carry over to our context Grušin's results on the partially Fourier-transformed operators. In this way we prove Lemma 4.3, and hence complete the proof of Theorem 1.2.

The plan of this section is as follows. First we shall discuss Grušin's results. Next we shall prove the above-mentioned equivalence, and, in particular, prove Lemma 4.3. We shall also find it instructive to compare the translation (via this equivalence) of Grušin's hypoellipticity criterion with Theorem 1.2. The equivalence between certain of the Grušin operators and operators on the Heisenberg group is very natural from the point of view of the representation theory of enveloping algebras of nilpotent Lie algebras. Essentially, it is the map introduced by Gabriel and Nouazé [19]. (See also [5, Chapitre 4, 87] and [4].) Although we have not attempted to prove this in general, we believe that by using other nilpotent Lie groups with dilations besides the Heisenberg group, one can probably obtain all the Grušin operators (though not vice versa) via such an equivalence. We shall present one simple example to illustrate the new phenomena which may arise. To conclude this section we shall sketch a possible method for proving Lemma 4.3 and, consequently, the essential parts of Grušin's results concerning the partially Fourier-transformed operators directly in the group-theoretic context. This method, which reduces everything to standard facts about elliptic operators, seems to us to offer one explanation of "why" these results hold.

Let $n, k$ be positive integers, and let $\delta$ be a positive rational number. The differential operators $P\left(t, D_{t}, D_{z}\right)$ considered by Grušin have polynomial coefficients in $t=\left(t_{1}, \ldots, t_{n}\right)$ and are constant-coefficient with respect to $z=\left(z_{1}, \ldots, z_{k}\right)$, and satisfy the following additional two properties.

(6.1) $P\left(t, D_{t}, D_{z}\right)$ is quasi-homogeneous of degree $m$.

That is, $p\left(t / r, r \tau, r^{(1+\delta)} \lambda\right)=r^{m} p(t, \tau, \lambda)$ for every $\tau \in \mathbf{R}^{n}, \lambda \in \mathbf{R}^{k}$ and $r>0$. Here $p(t, \tau, \lambda)$ is the total-symbol of $P\left(t, D_{t}, D_{z}\right)$, obtained by replacing $D_{t_{i}}=(1 / \sqrt{-1}) \partial / \partial t_{i}$ by $\tau_{i}$, and $D_{z_{j}}=(1 / \sqrt{-1}) \partial / \partial z_{j}$ by $\lambda_{j}$. 
(6.2) $P\left(t, D_{t}, D_{z}\right)$ is semielliptic. That is, the order of $P\left(t, D_{t}, D_{z}\right)$ as a differential operator is $m$, the integer appearing in (6.1), and $P\left(t, D_{t}, D_{z}\right)$ is elliptic when $t \neq 0$.

For $\lambda \in \mathbf{R}^{k}-\{0\}$, let $P\left(t, D_{t}, \lambda\right)$ be the partial Fourier transform of $P\left(t, D_{t}, D_{z}\right)$, obtained by replacing $D_{z_{j}}$ by $\lambda_{j}$. Let $H_{(m, \delta)}\left(\mathbf{R}^{n}\right)$ be defined as in Definition 4.1. Under the assumptions (6.1), (6.2) Grušin is able to prove the following additional properties.

(6.3) For every $\lambda \in \mathbf{R}^{k}-\{0\}$ the operator $P\left(t, D_{t}, \lambda\right)$, viewed as an operator from $H_{(m, \delta)}\left(\mathbf{R}^{n}\right)$ to $L^{2}\left(\mathbf{R}^{n}\right)$, is Fredholm, i.e., is bounded, has finite-dimensional kernel and cokernel, and has closed range.

(6.4) Let $\lambda \in \mathbf{R}^{k}-\{0\}$, and let $v(t)$ be a tempered distribution. Then if $P\left(t, D_{t}, \lambda\right) v$ lies in $L^{2}\left(\mathbf{R}^{n}\right)$ (respectively, $\delta\left(\mathbf{R}^{n}\right)$ ), $v$ lies in $H_{(m, \delta)}\left(\mathbf{R}^{n}\right)$ (respectively, $\delta\left(\mathbf{R}^{n}\right)$ ).

As an immediate corollary of (6.3) and (6.4) one obtains

(6.5) Let $\lambda \in \mathbf{R}^{k}-\{0\}$. Then the equation $P\left(t, D_{t}, \lambda\right) v=0$ has no nontrivial solution $v$ in $\delta\left(\mathbf{R}^{n}\right) \Leftrightarrow P\left(t, D_{t}, \lambda\right)$ has a bounded left-inverse $L$ : $L^{2}\left(\mathbf{R}^{n}\right) \rightarrow H_{(m, \delta)}\left(\mathbf{R}^{n}\right)$.

We can now state Grušin's hypoellipticity theorem:

(6.6) Let $P\left(t, D_{t}, D_{z}\right)$ satisfy (6.1) and (6.2). Then $P\left(t, D_{t}, D_{z}\right)$ is hypoelliptic $\Leftrightarrow$ for every $\lambda \in \mathbf{R}^{k}-\{0\}, P\left(t, D_{t}, \lambda\right)$ satisfies the equivalent conditions in (6.5).

It is easily seen that if $P\left(t, D_{t}, D_{z}\right)$ satisfies (6.1), and (6.2) then so does $P^{*}\left(t, D_{t}, D_{z}\right)$, the formal adjoint. Applying (6.4) to $P^{*}\left(t, D_{t}, D_{z}\right)$ and using the fact that $\delta\left(\mathbf{R}^{n}\right)$ is dense in $H_{(m, \delta)}\left(\mathbf{R}^{n}\right)$, we see that if $P\left(t, D_{t}, \lambda\right)$ is viewed as an unbounded operator on $L^{2}\left(\mathbf{R}^{n}\right)$ with domain $H_{(m, \delta)}\left(\mathbf{R}^{n}\right)$, then its Hilbertspace adjoint $\left[P\left(t, D_{t}, \lambda\right)\right]^{*}$ has domain $H_{(m, \delta)}\left(\mathbf{R}^{n}\right)$. Hence it follows that $\left[P\left(t, D_{t}, \lambda\right)\right]^{*}=P^{*}\left(t, D_{t}, \lambda\right)$, the partial Fourier transform of $P^{*}\left(t, D_{t}, D_{2}\right)$. In particular, we have

$$
\operatorname{Ker} P^{*}\left(t, D_{t}, \lambda\right)=\left[\text { Range } P\left(t, D_{t}, D_{z}\right)\right]^{\perp}
$$

and so

$$
\operatorname{dim} \operatorname{Ker} P^{*}\left(t, D_{t}, \lambda\right)=\operatorname{dim} \operatorname{Coker} P\left(t, D_{t}, \lambda\right) .
$$

We shall see that Lemma 4.3 is an immediate consequence of the following.

Lemma 6.1. Let $P\left(t, D_{t}, D_{z}\right)$ satisfy (6.1) and (6.2), and let $\lambda \in \mathbf{R}^{k}-\{0\}$. Then the following are equivalent.

(1) Neither of the equations $P\left(t, D_{t}, \lambda\right) v=0, P^{*}\left(t, D_{t}, \lambda\right) v=0$ has a nontrivial solution $v \in \mathcal{S}\left(\mathbf{R}^{n}\right)$.

(2) $P\left(t, D_{t}, \lambda\right)$ has a bounded two-sided inverse $L$, i.e., a bounded operator $L$ : $L^{2}\left(\mathbf{R}^{n}\right) \rightarrow L^{2}\left(\mathbf{R}^{n}\right)$ such that $L$ maps $\delta\left(\mathbf{R}^{n}\right)$ into $\delta\left(\mathbf{R}^{n}\right)$, and such that 
$P\left(t, D_{t}, \lambda\right) L=I$ on $\delta\left(\mathbf{R}^{n}\right)$ and $L P\left(t, D_{t}, \lambda\right)=I$ on $\delta\left(\mathbf{R}^{n}\right)$. (Clearly such an $L$, if it exists, is unique.)

If the equivalent conditions (1), (2) hold then $L$ satisfies the following additional properties:

(6.9) $L$ maps $L^{2}\left(\mathbf{R}^{n}\right)$ into $H_{(m, \delta)}\left(\mathbf{R}^{n}\right)$ and is bounded as an operator from $L^{2}\left(\mathbf{R}^{n}\right)$ into $H_{(m, \delta)}\left(\mathbf{R}^{n}\right)$.

(6.10) $P\left(t, D_{t}, \lambda\right) L=I$ on $L^{2}\left(\mathbf{R}^{n}\right)$.

(6.11) $L P\left(t, D_{t}, \lambda\right)=I$ on $H_{(m, \delta)}\left(\mathbf{R}^{n}\right)$.

(6.12) If $f \in L^{2}\left(\mathbf{R}^{n}\right)$ and $L f \in \mathcal{S}\left(\mathbf{R}^{n}\right)$, then $f \in \mathcal{S}\left(\mathbf{R}^{n}\right)$.

Proof. The second equation in $(2) \Rightarrow P\left(t, D_{t}, \lambda\right) v=0$ has no solution $v \neq 0$ in $\delta\left(R^{n}\right)$. Moreover, the first equation in $(2) \Rightarrow L^{*} P^{*}\left(t, D_{t}, \lambda\right)=I$ on $\delta\left(\mathbf{R}^{n}\right)$, and this in turn $\Rightarrow P^{*}\left(t, D_{l}, \lambda\right) v=0$ has no solution $v \neq 0$ in $\delta\left(\mathbf{R}^{n}\right)$. Thus (2) $\Rightarrow(1)$.

Suppose (1) holds. Then, in view of (6.3), (6.4), and (6.8), we see that $P\left(t, D_{t}, \lambda\right)$ has a bounded two-sided inverse $L: L^{2}\left(\mathbf{R}^{n}\right) \rightarrow H_{(m, \delta)}\left(\mathbf{R}^{n}\right)$, i.e., an operator satisfying (6.9)-(6.11). To show that (2) holds it suffices to prove that $L$ maps $\delta\left(\mathbf{R}^{n}\right)$ into $\delta\left(\mathbf{R}^{n}\right)$. But this follows from (6.10) and (6.4). Hence (1) $\Rightarrow(2)$.

Finally, (6.12) follows from (6.10) and the fact that $P\left(t, D_{t}, \lambda\right)$ maps $\delta\left(\mathbf{R}^{n}\right)$ into $\mathcal{S}\left(\mathbf{R}^{n}\right)$.

To discuss the equivalence result it is convenient to introduce some notation. The algebra (over $\mathbf{C}$ ) of differential operators in $n+k$ variables with polynomial coefficients in the first $n$ variables and constant coefficients in the last $k$ variables can be thought of as

$$
A_{n}\left(\mathbf{C}\left[D_{z_{1}}, \ldots, D_{z_{k}}\right]\right)=A_{n}(\mathrm{C}) \otimes_{\mathrm{C}} \mathbf{C}\left[D_{z_{1}}, \ldots, D_{z_{k}}\right],
$$

where $A_{n}(\mathrm{C})$ is the Weyl algebra, consisting of all differential operators on $\mathrm{R}^{n}$ with polynomial coefficients, and where $\mathrm{C}\left[D_{z_{1}}, \ldots, D_{z_{k}}\right]$ is the ring of polynomials in $D_{z_{1}}, \ldots, D_{z_{k}}$.

Now consider the case $k=1$ and $\delta=1$. Let $i$ : $U\left(h_{n}\right) \rightarrow A_{n}\left(\mathrm{C}\left[D_{z}\right]\right)$ be the homomorphism determined by the Lie algebra homomorphism: $h_{n} \rightarrow$ $A_{n}\left(\mathrm{C}\left[D_{z}\right]\right)$ given by

$$
X_{i} \mapsto d / d t_{i}, \quad Y_{i} \mapsto i t_{i} D_{z}, \quad Z \mapsto i D_{z} .
$$

Proposition 6.2. The map $i$ : $U\left(h_{n}\right) \rightarrow A_{n}\left(\mathrm{C}\left[D_{z}\right]\right)$ satisfies the following properties:

(1) $P \in \mathcal{U}\left(h_{n}\right)$ is homogeneous of degree $m \Leftrightarrow i(P)$ is quasi-homogeneous of degree $m$.

(2) Let $P \in \mathcal{Q}\left(h_{n}\right)$ be homogeneous. Then $\pi_{(\xi, \eta)}(P) \neq 0$ for every $(\xi, \eta) \in$ $\mathbf{R}^{2 n}-\{0\} \Leftrightarrow i(P)$ is semielliptic.

(3) For any $\lambda \in \mathbf{R}-\{0\}$ let $\pi_{\lambda}$ denote the irreducible unitary representation 
of $H_{n}$ defined by (3.9) and (3.10). Then for any $P \in \mathcal{Q}\left(h_{n}\right), \pi_{\lambda}(P)=$ the partial Fourier transform of $i(P)$ evaluated at $\lambda$.

(4) The map $i$ is injective, and range $i$ contains all operators in $A_{n}\left(\mathbf{C}\left[D_{z}\right]\right)$ which are quasi-homogeneous (with $\delta=1$ ) and semielliptic.

Proof. Using multi-index notation we can express $P \in \mathcal{U}\left(h_{n}\right)$ as

$$
P=\sum_{|\alpha|+|\beta|+\gamma<k} a_{\alpha \beta \gamma} Y^{\alpha} X^{\beta} Z^{\gamma}, \quad a_{\alpha \beta \gamma} \in \mathbf{C} .
$$

We can, of course, assume that for some $\alpha \beta \gamma$ with $|\alpha|+|\beta|+\gamma=k$, $a_{\alpha \beta \gamma} \neq 0$. It follows from (6.14) that

(6.15) $P$ is homogeneous of degree $m \Leftrightarrow|\alpha|+|\beta|+2 \gamma=m$ for every $\alpha \beta \gamma$ such that $a_{\alpha \beta \gamma} \neq 0$.

But

$$
i(P)=\sum_{|\alpha|+|\beta|+\gamma<k}(i)^{|\alpha|+|\beta|+\gamma} a_{\alpha \beta \gamma} t^{\alpha} D_{i}^{\beta} D_{z}^{|\alpha|+\gamma},
$$

and so the total symbol of $i(P)$ is given by

$$
p(t, \tau, \lambda)=\sum_{|\alpha|+|\beta|+\gamma<k}(i)^{|\alpha|+|\beta|+\gamma} a_{\alpha \beta \gamma} t^{\alpha} \tau^{\beta} \lambda^{|\alpha|+\gamma} .
$$

It follows immediately from (6.17) that

(6.18) $i(P)$ is quasi-homogeneous of degree $m$ (with $\delta=1) \Leftrightarrow-|\alpha|+$ $|\beta|+2(|\alpha|+\gamma)=m$ for every $\alpha \beta \gamma$ such that $a_{\alpha \beta \gamma} \neq 0$.

But this is precisely the condition in (6.15). This proves (1).

Suppose next that $P$, given by (6.14), is homogeneous of degree $m$. Arguing exactly as in the proof of Lemma 3.8 we see

$$
\begin{gathered}
\pi_{(\xi, \eta)}(P) \neq 0 \text { for every }(\xi, \eta) \in \mathbf{R}^{2 n}-\{0\} \Leftrightarrow k=m \text { and } \\
\sum_{|\alpha|+|\beta|=m} a_{\alpha \beta 0} \xi^{\alpha} \eta^{\beta} \neq 0 \text { for every }(\xi, \eta) \in \mathbf{R}^{2 n}-\{0\} .
\end{gathered}
$$

But by the definition, (6.2), of semiellipticity; and from (6.16), we get

(6.20) $i(P)$ is semielliptic $\Leftrightarrow k=m$ and

$$
\sum_{|\alpha|+|\beta|=m}(i)^{|\alpha|+|\beta|} a_{\alpha \beta 0} t^{\alpha} \tau^{\beta} \lambda^{|\alpha|} \neq 0 \text { for every } t \in \mathbf{R}^{n}-\{0\},
$$

$$
(\tau, \lambda) \in \mathbf{R}^{n+1}-\{0\} .
$$

Noting that $|\alpha|+|\beta|=m$ for every $a_{\alpha \beta 0}$ occurring in the above, we see that we can rewrite the above condition as

$$
\begin{aligned}
\sum_{|\alpha|+|\beta|=m} a_{\alpha \beta 0}(\lambda t)^{\alpha} \tau^{\beta} \neq 0 \text { for every } t \in \mathbf{R}^{n}-\{0\} & \\
& (\tau, \lambda) \in \mathbf{R}^{n+1}-\{0\} .
\end{aligned}
$$


It then follows immediately that the conditions given by (6.19) and (6.20) are identical. This proves (2).

(3) is obvious from the definition of $i$. Also, the injectivity of $i$ is obvious from (6.16). The surjectivity asserted in (4) follows easily from the fact (contained in (6.2)) that the order of a semielliptic, quasi-homogeneous differential operator is equal to its homogeneity degree.

Since $\tilde{\pi}_{1}=\pi_{1}$ and $\tilde{\pi}_{-1}=\pi_{-1}$, we see that Lemma 4.3 follows immediately from Lemma 6.1 via the equivalence given by Proposition 6.2.

We next wish to place the above equivalence in the framework of Gabriel and Nouazé [19]. Let $G$ be a simply-connected nilpotent Lie group of dimension $N$. Let $\mathscr{U}(g), Z(g), n, q$ be as defined in Theorem 2.7. We can think of $Z(g)$, the center of the enveloping algebra, as consisting of the bi-invariant, i.e., commuting both with left and right translation, linear differential operators on $G$. Let Fract $Z(g)$ denote the field of fractions of $Z(g)$, and consider the ring $\mathcal{U}(g) \otimes_{Z(g)}$ Fract $Z(g)$. This can be thought of as all formal fractions $P / Q$, where $P$ is a left-invariant and $Q$ a bi-invariant differential operator on $G$. It is possible to view the fractions $P / Q$ as actual analytic objects, in fact as distributions on $G$. In fact, by a theorem of Rais [20], every bi-invariant $Q$ has a fundamental solution $v_{Q} \in \mathscr{Q}^{\prime}(G)$, the dependence of $v_{Q}$ on $Q$ being very regular. In particular, the map $P / Q \mapsto$ $(P \delta) * v_{Q}$ is well-defined.

Let $\mathbf{C}\left(D_{z_{1}}, \ldots, D_{z_{q}}\right)$ denote the ring of rational functions in the commuting indeterminates $D_{z_{1}}, \ldots, D_{z_{q}}$, and let $A_{n}\left(C\left(D_{z_{1}}, \ldots, D_{z_{q}}\right)\right)$ denote the ring $A_{n}(\mathrm{C}) \otimes_{\mathrm{C}} \mathrm{C}\left(D_{z_{1}}, \ldots, D_{z_{q}}\right)$. Gabriel and Nouazé show

(6.22) $U(g) \otimes_{Z(g)}$ Fract $Z(g)$ is isomorphic to $A_{n}\left(\mathrm{C}\left(D_{z_{1}}, \ldots, D_{z_{q}}\right)\right)$, where $n$ and $q$ are the integers occurring in Theorem 2.7.

Let $I$ be a two-sided prime ideal in $U(g)$. Let $\mathcal{U}(g ; I)$ denote $U(g) / I$, $Z(g ; I)$ the center of $\mathcal{U}(g ; I)$, and Fract $Z(g ; I)$ the field of fractions of $Z(g ; I)$. Then Gabriel and Nouazé show further that

(6.23) $\mathcal{Q}\left(g_{;} I\right) \otimes_{Z\left(g_{;} I\right)}$ Fract $Z(g ; I)$ is isomorphic to $A_{m}\left(\mathbf{C}\left(D_{z_{1}}, \ldots, D_{z}\right)\right)$ for some integers $m, l$.

In the case $G=H_{n}$ we noted in the remarks following (3.10) that $Z(g)$ consists precisely of the polynomials in $Z$, so that $q=1$. We thus obtain the diagram

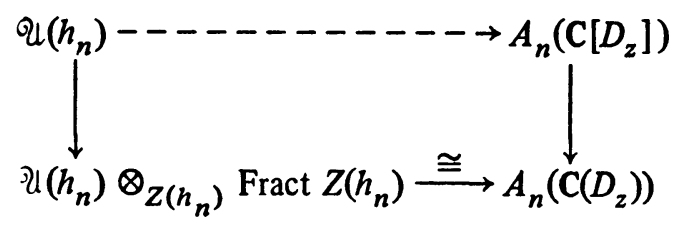

where the vertical arrows denote inclusion maps, and the bottom horizontal arrow denotes the isomorphism in (6.22). The map $i$ of Proposition 6.2 
appears here as the (unique) map which fills in the top line. Thus the Grušin operators with $n$ arbitrary, $k=1$, and $\delta=1$ can be related naturally to homogeneous operators on $H_{n}$ via the equivalence of Proposition 6.2 and the Gabriel-Nouazé isomorphism in (6.22).

It is interesting to observe that the situation can become more complicated for other Grušin operators. Indeed the lowest dimensional nilpotent Lie group analogously related to the Grušin operators with $n=1, k=2, \delta=1$ is of dimension 5 rather than $4=2 n+k$, and the more general Gabriel-Nouazé isomorphism, given by (6.23), is needed. Since the results treat only a special example we shall omit the proofs and merely sketch the pertinent facts. The source of the example is Dixmier's exhaustive treatment [3] of the simplyconnected nilpotent Lie groups of dimension $\leqslant 5$.

The Lie algebra $g_{5,2}$ of the group in question, $G_{5,2}$, has generators $X_{1}, X_{2}$, $X_{3}, X_{4}, X_{5}$ whose only nonzero commutation relations are

$$
\left[X_{1}, X_{2}\right]=X_{4}, \quad\left[X_{1}, X_{3}\right]=X_{5} \text {. }
$$

Thus it has a natural family of dilations given by

$$
\begin{gathered}
\delta_{r}\left(X_{1}\right)=r X_{1}, \delta_{r}\left(X_{2}\right)=r X_{2}, \delta_{r}\left(X_{3}\right)=r X_{3}, \\
\delta_{r}\left(X_{4}\right)=r^{2} X_{4}, \delta_{r}\left(X_{5}\right)=r^{2} X_{5} .
\end{gathered}
$$

The center of $\mathcal{U}\left(g_{5,2}\right)$ is given by

$$
Z\left(g_{5,2}\right)=\mathrm{C}\left[X_{4}, X_{5}, X_{2} X_{5}-X_{3} X_{4}\right],
$$

the three generators being algebraically independent.

Let $i$ : $\mathcal{U}\left(g_{5,2}\right) \rightarrow A_{1}\left(\mathrm{C}\left[D_{z_{1}}, D_{z_{2}}\right]\right)$ be the homomorphism determined by the Lie algebra homomorphism: $g_{5,2} \rightarrow A_{1}\left(\mathrm{C}\left[D_{z_{1}}, D_{z_{2}}\right]\right.$ given by

$$
\begin{gathered}
X_{1} \mapsto d / d t, \quad X_{2} \mapsto i t D_{z_{1}}, \quad X_{3} \mapsto i t D_{z_{2}}, \\
X_{4} \mapsto i D_{z_{1}}, \quad X_{5} \mapsto i D_{z_{2}} .
\end{gathered}
$$

Notice that $i$ maps the third generator $X_{2} X_{5}-X_{3} X_{4}$ of $Z\left(g_{5,2}\right)$ to 0 . The following results are easily proved:

(6.29) The kernel, $I$, of $i$, which is a prime ideal in $U\left(g_{5,2}\right)$, is the (two-sided) ideal in $थ\left(g_{5,2}\right)$ generated by $X_{2} X_{5}-X_{3} X_{4}$.

(6.30) For this ideal $I$ the isomorphism of (6.23) takes the form

$$
\mathcal{U}\left(g_{5,2 ;}, I\right) \otimes_{Z\left(8_{5,2 ;} l\right)} \text { Fract } Z\left(g_{5,2 ;} I\right) \cong A_{1}\left(\mathrm{C}\left(D_{z_{1}}, D_{z_{2}}\right)\right) \text {, }
$$

and the diagram

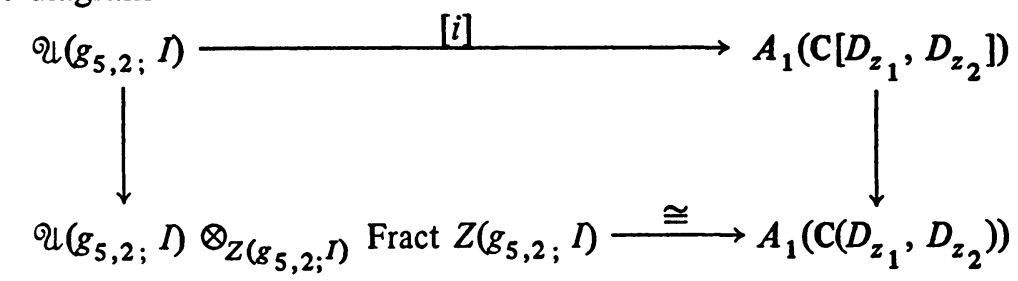


commutes, where $[i]$ is the quotient through $I$ of $i$.

The analogue of Proposition 6.2 holds, but, as one might expect from (6.29), we do not need to consider all the irreducible unitary representations of $G_{5,2}$, but only those which map $X_{2} X_{5}-X_{3} X_{4}$ to 0 . As is shown in [3], $G_{5,2}$ has a generic family $\pi_{\lambda, \mu, \nu}$ of irreducible unitary representations acting on $L^{2}(\mathbf{R})$, parametrized by $(\lambda, \mu, \nu) \in\left(\mathbf{R}^{2}-\{0\}\right) \times \mathbf{R}$, which map $X_{4}$ to $i \lambda, X_{5}$ to $i \mu$, and $X_{2} X_{5}-X_{3} X_{4}$ to $\nu$, and a "degenerate" family of 1-dimensional representations which map all of $Z\left(g_{5,2}\right)$ to 0 . The representations which map $X_{2} X_{5}-X_{3} X_{4}$ to 0 clearly give representations of $\mathcal{U}\left(g_{5,2 ;} I\right)$. Notice also that, since $X_{2} X_{5}-X_{3} X_{4}$ is homogeneous with respect to the dilations $\left\{\delta_{r}\right\}$, each $\delta_{r}$ maps $I$ into $I$, and so induces an automorphism of $\mathcal{U}\left(g_{5,2 ;} I\right)$, and similarly for multiplication by $r \in \mathbf{R}^{+}$. It is therefore meaningful to say that a given element of $U\left(g_{5,2 ;} I\right)$ is homogeneous of degree $m$. Letting $[P]$ denote the equivalence class in $\mathcal{U}\left(g_{5,2}, I\right)$ of $P \in \mathcal{Q}\left(g_{5,2}\right)$, we can state the following analogue of Proposition 6.2.

Proposition 6.3. The map [i]: $U\left(g_{5,2 ;} I\right) \rightarrow A_{1}\left(\mathrm{C}\left[D_{z_{1}}, D_{z_{2}}\right)\right.$ satisfies the following properties:

(1) $[P] \in \mathcal{U}\left(g_{5,2}, I\right)$ is homogeneous of degree $m \Leftrightarrow[i]([P])$ is quasi-homogeneous of degree $m$.

(2) Let $[P] \in \mathcal{U}\left(g_{5,2}, I\right)$ be homogeneous. Then $\pi([P] \neq 0$ for every "degenerate" representation $\pi$ of $G_{5,2}$ (except the trivial, identity representation) $\Leftrightarrow[i]([P])$ is semielliptic.

(3) For every $(\lambda, \mu) \in \mathbf{R}^{2}-\{0\}$ and for any $P \in \mathcal{Q}\left(g_{5,2}\right), \pi_{\lambda, \mu, 0}([P])=$ the partial Fourier transform of $[i]([P])$ evaluated at $(\lambda, \mu)$.

(4) The map [i] is injective, and the range contains all operators in $A_{1}\left(C\left[D_{z_{1}}\right.\right.$, $D_{z_{2}}$ l) which are quasi-homogeneous (with $\delta=1$ ) and semielliptic.

As we stated earlier, the proof will be omitted.

Returning for a moment to the equivalence provided by Proposition 6.2, we see that Grušin's hypoellipticity theorem (6.6) "translates" to the following statement on the Heisenberg group:

(6.6') Let $P \in \mathcal{Q}\left(h_{n}\right)$ be homogeneous, and suppose that $\pi_{(\xi, \eta)}(P) \neq 0$ for every $(\xi, \eta) \in \mathbf{R}^{2 n}-\{0\}$. Then $P$ is hypoelliptic if and only if for every $\lambda \in \mathbf{R}-\{0\}$ the equation $\tilde{\pi}_{\lambda}(P) v=0$ has no nonzero solution $v \in \mathcal{S}\left(\mathbf{R}^{n}\right)$.

I do not know whether $\left(6.6^{\prime}\right)$ states a true result, though I suspect that it does. The Grušin operators and the group operators live on manifolds of different dimensions, and I do not see how to show that passage from one domain to the other preserves hypoellipticity.

I shall conclude this section with a sketch of a possible method for proving a variant of Lemma 4.3 directly in the group-theoretic setting. The method 
may be useful in trying to prove Theorem 1.2 for other simply-connected nilpotent Lie groups $G$ with dilations.

The hypothesis of Lemma 4.3 is that $P \in \mathcal{Q}\left(h_{n}\right)$ is homogeneous, and that $\pi_{(\xi, \eta)}(P) \neq 0$ for every $(\xi, \eta) \in \mathbf{R}^{2 n}-\{0\}$. As we saw earlier (see, for example, Proposition 3.7) this allows us to assume, without loss of generality, that there exists $T \in Z\left(h_{n}\right)$ such that $P+T$ is elliptic, though not homogeneous.

We pass now to the general context of a simply-connected nilpotent Lie group $G$ with dilations, and shall freely use the results and notations of $\$ 2$. Recall (see the remarks preceding Lemma 2.1, and Note 1 following Proposition 2.6) that the Gårding subspace of $\pi_{\lambda}, \lambda \in \Gamma$, is defined as the subspace of $L^{2}\left(\mathbf{R}^{n}\right)$ spanned by $\left\{\pi_{\lambda}(\varphi) v \mid \varphi \in C_{0}^{\infty}(G), v \in L^{2}\left(\mathbf{R}^{n}\right)\right\}$. It is contained in $\delta\left(\mathbf{R}^{n}\right)$, is dense in $L^{2}\left(\mathbf{R}^{n}\right)$, and is preserved by $\pi_{\lambda}(u)$ for any $u \in \mathcal{E}^{\prime}(G)$. In what follows we could actually work with any irreducible unitary representation of $G$, not just the generic representations, but we shall restrict attention to these.

We assume that $P \in \mathcal{Q}(g)$ and that there exists $T \in Z(g)$ such that $P+T$ is elliptic. (In practice, $P$ will be homogeneous, and $T$ will be obtained as in the case of $G=H_{n}$ above.) Fix $\lambda \in \Gamma$. Since $\pi_{\lambda}$ is irreducible and $T \in Z(g), \pi_{\lambda}(T)$ is a scalar, i.e., $\pi_{\lambda}(T)=c_{\lambda}$ for some $c_{\lambda} \in \mathbf{C}$.

Letting $Q=P+T-c_{\lambda}$, we see that $Q$ (hence, $Q^{\prime}$ ) is elliptic, and that $\pi_{\lambda}(Q)=\pi_{\lambda}(P)$. Since $Q^{t}$ is elliptic there exists $u_{1} \in \mathcal{E}^{\prime}(G)\left(C^{\infty}\right.$ away from $e)$ and $f_{1} \in C_{0}^{\infty}(G)$ such that

$$
Q^{\prime} u_{1}=\delta+f_{1}
$$

Applying $\pi_{\lambda}$ to both sides we obtain

$$
\pi_{\lambda}\left(u_{1}\right) \pi_{\lambda}(P)=I+\pi_{\lambda}\left(f_{1}\right)
$$

Working with $Q^{*}$ instead of $Q$ we obtain $u_{2}, f_{2}$ such that

$$
\pi_{\lambda}\left(u_{2}\right) \pi_{\lambda}(P)^{*}=I+\pi_{\lambda}\left(f_{2}\right) \text {. }
$$

Equations (6.33) and (6.34) can be viewed as equations between operators on the $C^{\infty}$-vectors, $\delta\left(\mathbf{R}^{n}\right)$, or on the Gårding subspace.

A result of Nelson and Stinespring (see [18, Theorem 2.2D) applied to $\pi_{\lambda}(Q)$, and hence to $\pi_{\lambda}(P)$, shows

(6.35) If $\pi_{\lambda}(P)$ and $\pi_{\lambda}(P)^{*}$ are viewed as densely-defined operators on $L^{2}\left(\mathbf{R}^{n}\right)$ with domain the Gårding subspace, then the Hilbert-space closure of $\pi_{\lambda}(P)$ is the Hilbert-space adjoint of $\pi_{\lambda}(P)^{*}$ and vice versa. Since both $\pi_{\lambda}(P)$ and $\pi_{\lambda}(P)^{*}$ are differential operators with polynomial coefficients it is easy to see that $\delta\left(\mathbf{R}^{n}\right)$ is contained in the domains of the closures.

Let $\bar{H}$ denote the domain of the closure of $\pi_{\lambda}(P)$. It is a Hilbert space with respect to the norm defined by

(6.36) $\|v\|\|=\| v\|+\| \pi_{\lambda}(P) v \|$, where \|\| is the $L^{2}$-norm. 
Applying (6.35) we obtain the following analogue of (6.4).

(6.37) If $v \in L^{2}\left(\mathbf{R}^{n}\right)$ and $\pi_{\lambda}(P) v \in L^{2}\left(\mathbf{R}^{n}\right)$, then $v \in \bar{H}$.

Since $f_{1} \in C_{0}^{\infty}(G), \pi_{\lambda}\left(f_{1}\right)$ maps $L^{2}\left(\mathbf{R}^{n}\right)$ into the Gårding subspace. Hence it follows from (6.33), (6.37), and Note 6 following Proposition 2.6 that

(6.38a) If $v \in L^{2}\left(\mathbf{R}^{n}\right)$ and $\pi_{\lambda}(P) v \in \mathcal{S}\left(\mathbf{R}^{n}\right)$, then $v \in \mathcal{S}\left(\mathbf{R}^{n}\right)$.

(6.38b) If $v \in L^{2}\left(\mathbf{R}^{n}\right)$ and $\pi_{\lambda}(P) v \in$ Gårding subspace, then $v \in$ Gårding subspace. In particular, $\operatorname{Ker} \pi_{\lambda}(P) \subseteq$ Gårding subspace, a fortiori in $\mathcal{S}\left(\mathbf{R}^{n}\right)$.

Now, since $f_{1} \in C_{0}^{\infty}(G), \pi_{\lambda}\left(f_{1}\right)$ is of trace-class, in particular, compact, and so $I+\pi_{\lambda}\left(f_{1}\right)$ is Fredholm. Hence from (6.33) and the above it follows that

(6.39) $\operatorname{Ker} \pi_{\lambda}(P)$ is a finite-dimensional subspace of the Gårding subspace.

Working with (6.34) we find

(6.40) [Range $\left.\pi_{\lambda}(P)\right]^{\perp}$ is a finite-dimensional subspace of the Gårding subspace.

By the definition of $\bar{H}$ we know that the map $\pi_{\lambda}(P): \bar{H} \rightarrow L^{2}\left(\mathbf{R}^{n}\right)$ is continuous. If we could show that it has closed range then it would follow from (6.39) and (6.40) that $\pi_{\lambda}(P)$ is Fredholm. To prove the range is closed it would, for example, suffice to show that the inclusion map: $\bar{H} \rightarrow L^{2}\left(\mathbf{R}^{n}\right)$ is compact rather than just continuous. We have not succeeded in proving this, but we suspect that it is true, since the norm on $\bar{H}$ is defined in terms of $\pi_{\lambda}(P)=\pi_{\lambda}(Q)$, where $Q$ is elliptic. Furthermore, since $Q$ is elliptic, the parametrices $u_{1}, u_{2}$ have strong boundedness properties as maps between Sobolev spaces, and so it may be possible, in view of (6.33) and (6.34), to prove the following analogue of Lemma 4.2.

(6.41) Suppose that $P$ given above is homogeneous of degree $m$. Then for any $R \in \mathcal{U}(g)$ homogeneous of degree $\leqslant m, \pi_{\lambda}(R)$ extends to a bounded map: $\bar{H} \rightarrow L^{2}\left(\mathbf{R}^{n}\right)$.

(6.35) implies that if $P=P^{*}$, then the formally selfadjoint operator $\pi_{\lambda}(P)$ has a unique selfadjoint extension, namely the Hilbert-space closure of $\pi_{\lambda}(P)$. In particular, if $P \in \mathcal{U}\left(h_{n}\right)$ is formally selfadjoint and homogeneous of degree $m$, and satisfies (3.57) and (3.59), it follows, in conjunction with the remarks preceding (6.7), that $\bar{H}=H_{(m, 1)}\left(\mathbf{R}^{n}\right)$. At least for $m$ even, when we know such operators $P$ exist, this provides a type of abstract characterization of $H_{(m, 1)}\left(\mathbf{R}^{n}\right)$.

7. A class of examples. As an illustration of Theorem 1.2 we present the following variant of Proposition 1.3:

Proposition 7.1. Let $G$ be a simply-connected nilpotent Lie group, with Lie algebra $g$. Let $X_{1}, \ldots, X_{k}$ be elements of $g$ whose repeated commutators span $g$, and let $R \in \mathcal{Q}(g)$ be skew-adjoint, i.e., $R^{*}=-R$. Then for any positive integers $m_{i}, i=1, \ldots, k$, all even or all odd, the operator 


$$
P=\sum_{i=1}^{k} X_{i}^{2 m_{i}}+R
$$

satisfies the following property:

(7.2) For every irreducible unitary representation $\pi$ of $G$ (except the trivial, identity representation) $\pi(P) v \neq 0$ and $\pi(P)^{*} v \neq 0$ for every nonzero $C^{\infty}$. vector $v$ of the representation $\pi$.

Notice, in particular, that any $X_{0} \in g$ could be taken as $R$.

Proof. Since $P^{*}=\sum_{i=1}^{k} X_{i}^{2 m}-R$, and since $-R$ is also skew-adjoint, it is enough to show that $\pi(P) v \neq 0$ if $v \neq 0$. Since $\pi(X)^{*}=-\pi(X)$ for every $X \in g$ and since all the $m_{i}$ have the same parity, it follows that

$$
\langle\pi(P) v, v\rangle=(-1)^{m_{i}} \sum_{i=1}^{k}\left\langle\pi\left(X_{i}\right)^{m_{i}} v, \pi\left(X_{i}\right)^{m_{i}} v\right\rangle+\langle\pi(R) v, v\rangle
$$

for every $C^{\infty}$-vector $v$. The first term on the right-hand side of (7.3) is real. But since $R^{*}=-R$ it follows from (2.12) that

$$
\langle\pi(R) v, v\rangle=\left\langle v, \pi\left(R^{*}\right) v\right\rangle=-\langle v, \pi(R) v\rangle=-\overline{\langle\pi(R) v, v\rangle} .
$$

Thus $\langle\pi(R) v, v\rangle$ is imaginary, and so, by (7.3), if $\pi(P) v=0$, then $\left.\sum_{i=1}^{k}\left\langle\pi\left(X_{i}\right)^{m_{i}}, \pi\left(X_{i}\right)^{m_{i}}\right\rangle\right\rangle=0$. Each summand is nonnegative. Hence $\left\langle\pi\left(X_{i}\right)^{m_{i}}, \pi\left(X_{i}\right)^{m_{i}} v\right\rangle=0$ for every $i=1, \ldots, k$, and so

$$
\pi\left(X_{i}\right)^{m_{i}} v=0 \text { for every } i=1, \ldots, k .
$$

If $m_{i}$ is even, then (7.5) yields

$$
0=\left\langle\pi\left(X_{i}\right)^{m_{i}} v, v\right\rangle=(-1)^{m_{i} / 2}\left\langle\pi\left(X_{i}\right)^{m_{i} / 2} v, \pi\left(X_{i}\right)^{m_{i} / 2} v\right\rangle,
$$

and so $\pi\left(X_{i}\right)^{m_{i} / 2} v=0$. If $m_{i}$ is odd we work with $m_{i}+1$. Since $m_{i}+1<2 m_{i}$ unless $m_{i}=1$, we can iterate this process to obtain

$$
\pi\left(X_{i}\right) v=0 \text { for every } i=1, \ldots, k \text {. }
$$

Since $\pi$ is a Lie algebra homomorphism, and since the repeated commutators of $X_{1}, \ldots, X_{k}$ span $g$, it follows from (7.7) that

$$
\pi(X) v=0 \text { for every } X \in g .
$$

If the irreducible unitary representation $\pi$ of $G$ is infinite-dimensional then we know from [15] that, up to unitary equivalence (which takes $C^{\infty}$-vectors to $C^{\infty}$-vectors), we may assume that $\pi$ acts on $L^{2}\left(\mathbf{R}^{n}\right)$ for some $n$, that the space of $C^{\infty}$-vectors is $\delta\left(R^{n}\right)$, and that the range of $\pi$ as a representation of $U(g)$ is $A_{n}(\mathbf{C})$, the algebra of all differential operators on $\mathbf{R}^{n}$ with polynomial coefficients. Hence it follows from (7.8) that for every $Q \in A_{n}(\mathrm{C})$ there is a constant $c \in \mathbf{C}$ such that $(Q-c) v(t)=0$. In particular, there exist constants $c_{i}, i=1, \ldots, n$, such that

$$
\partial v(t) / \partial t_{i}=c_{i} v(t)
$$


Hence

$$
\left(\partial / \partial t_{i}\right)\left[\exp \left(-\sum_{j=1}^{n} c_{j} t_{j}\right) \cdot v(t)\right]=0, \quad i=1, \ldots, n,
$$

and so $v(t) \equiv C \exp \left(\sum_{j=1}^{n} c_{j} t_{j}\right)$ for some constant $C$. Since $v \in \delta\left(\mathbf{R}^{n}\right)$ it follows that $v=0$.

If the irreducible unitary representation $\pi$ is finite-dimensional (and therefore, by [15], 1-dimensional), it follows that if (7.8) holds for some $v \neq 0$, then $\pi(X)=0$ for every $X \in g$. Hence, since exp: $g \rightarrow G$ is bijective and since $\pi(\exp X)=\exp \pi(X)$ for every $X \in g$, it follows that $\pi$ is the 1-dimensional identity representation.

At least in the case of $G=H_{n}$ it follows from Theorem 1.2 that such $P$, if homogeneous, are hypoelliptic. In particular, this proves Corollary 1.4.

8. Open problems. We would like to conclude this paper by briefly discussing a few open problems.

One very interesting open question is whether Theorem 1.2 is valid in the case of a general simply-connected nilpotent Lie group $G$ with dilations $\left\{\delta_{r}\right\}$, and, if not, what restrictions on $G$ or $\left\{\delta_{r}\right\}$ are necessary. As is apparent from the treatment given here, much of the apparatus of the proof for $G=H_{n}$ is quite ready to be carried over to the general context. There are, however, substantial difficulties in the general case. We give two. (1) The lower-dimensional subvarieties of "degenerate" representations in $\mathrm{g}^{*}$ may be troublesome. For example, the unit "ball" in $\Gamma$, the space of generic orbits, will in general not be compact (unlike the case of $H_{n}$, where it consists of two points). (2) One cannot expect the analogue of Proposition 3.7 with $Z^{2 m}$ replaced by some $T \in Z(g)$ to hold generally. For example, take $G=H_{n}$ with the nonstandard choice of dilations $\delta_{r}: X_{i} \mapsto r^{3} X_{i}, Y_{i} \mapsto r^{2} Y_{i}, Z \mapsto r^{5} Z$, and let $P=\sum_{i=1}^{n} X_{i}^{2}+Y_{i}^{3}$. Of course, there are cases where the analogue of Proposition 3.7 does hold, for example $G=\mathbf{R}^{n}$ with any choice of dilations, and all simply-connected nilpotent Lie groups of dimension $<5$, as listed in [3], if we make the simplest choices of dilations for these groups. We note also that Theorem 1.2 holds for $G=\mathbf{R}^{n}$ with general dilations (see [13, Theorem 4.1.8]).

Another interesting question concerns the case of nonhomogeneous $P \in$ $\mathcal{U}\left(h_{n}\right)$. Certainly any $P \in \mathcal{U}\left(h_{n}\right)$ can be expressed uniquely as $P=P_{m}+$ $P_{m-1}+\cdots+P_{0}$, where $P_{j} \in \mathcal{Q}\left(h_{n}\right)$ is homogeneous of degree $j$ for each integer $j$. The analogue of ellipticity for $P$ would be the assumption that $P_{m}$, the top-order part, satisfies hypothesis (1.2) of Theorem 1.2. Is it true that if $P$ is "elliptic" in this sense, then $P$ is hypoelliptic and locally solvable? Can one construct a parametrix? Preliminary indications appear to be affirmative, and 
a procedure involving "symbols" with asymptotic expansions seems like it can be carried through. It is too early, however, to report further on this.

A third question concerns the local solvability, as opposed to hypoellipticity, of homogeneous $P \in \mathcal{Q}\left(h_{n}\right)$. for $P$ to be locally solvable is it sufficient that $P$ satisfy hypothesis (1.2), but now only for the generic representations? Is it possible, for example, by making use of (3.17)-(3.19) in conjunction with the Plancherel formula, to prove this by methods analogous to those used by Bernštein [1] to obtain fundamental solutions for constant-coefficient operators on $\mathbf{R}^{n}$ ?

One of the main reasons for studying homogeneous operators on nilpotent Lie groups is the hope that these operators might be used to approximate locally or microlocally, i.e., locally in the cotangent space, more general "variable-coefficient" differential or pseudo-differential operators on a manifold $M$. (See, for example, [6], [7], [21], [22].) We wish to discuss the results in [21] from this standpoint. Roughly speaking, the situation is as follows. $P$ is a pseudo-differential operator on a manifold $M$ such that the characteristic variety $\Sigma$ of $P$ is nondegenerate with respect to the symplectic form $\omega$ on $T^{*} M \backslash 0$, and such that the principal symbol of $P$ vanishes exactly to second order on $\Sigma$. For each $(x, \xi) \in \Sigma$, let $N(\Sigma)_{(x, \xi)}$ denote the fiber over $(x, \xi)$ of the conormal bundle $N(\Sigma)$ of $\Sigma$. The $2 n+1$-dimensional vector space $N(\Sigma)_{(x, \xi)} \oplus \mathbf{R}$ can be canonically identified with $h_{n}$ if we define a Lie bracket by $\left[\left(v_{1}, r_{1}\right),\left(v_{2}, r_{2}\right)\right]=\left(0, \omega\left(v_{1}, v_{2}\right)\right)$. Using this identification we can obtain from the principal and subprincipal parts of $P$ an element of $S\left(h_{n}\right)$, the symmetric algebra. Applying the symmetrization map: $S\left(h_{n}\right) \rightarrow \mathcal{U}\left(h_{n}\right)$ we get a homogeneous operator $P_{(x, \xi)}$ on $H_{n}$. The remarkable fact is that $P$ is hypoelliptic with loss of 1 derivative (a sharp form of hypoellipticity) if and only if $P_{(x, \xi)}$ is hypoelliptic for every $(x, \xi) \in \Sigma$. Especially in light of Theorem 1.2, it would be extremely interesting to know whether an analogous result can be formulated and proved for operators $P$ vanishing to order $>2$ on $\Sigma$. Even in the case of second-order vanishing we would very much like to have a more fundamental understanding of this group-theoretic "approximation" process.

ADDED IN PROOF. Since this paper was written some further progress has been made:

(1) For the Heisenberg group itself, we have found it is possible to avoid reference to the results of Grušin in $\$ 6$ and to work completely in the context of representation theory, by using the "lattice realizations" of the representations (see Cartier [27]). This has also been observed, independently, by $\mathrm{L}$. Corwin and R. Goodman, I believe.

(2) The necessity part of Theorem 1.2 (in the sharper form $\left(6.6^{\prime}\right)$ ) is true for arbitrary simply-connected nilpotent Lie groups $G$ with dilations (see Beals 
[25]). We have also proved this independently.

(3) Theorem 1.2 (in the sharper form $\left(6.6^{\prime}\right)$ ) is true for any two-step simply-connected nilpotent Lie group $G$ with the standard dilations. This has been shown by Helffer [28] and Beals [26], each using a different symbolcalculus. They also treat nonhomogeneous $P$, as discussed in $\S 8$, and construct parametrices. Their methods are quite different from those of the present paper, however, and do not make use of the Plancherel formula.

\section{REFERENCES}

1. I. N. Bernštein, Analytic continuation of generalized functions with respect to a parameter, Funkcional. Anal. i Priložen. 6 (1972), no. 4, 26-40 = Functional Anal. Appl. 6 (1972), no. 4, 273-285. MR 47 \#9269.

2. J. Dixmier, Sur les représentations unitaires des groupes de Lie nilpotents. II, Bull. Soc. Math. France 85 (1957), 325-388. MR 20 \# 1928.

3. , Sur les représentations unitaires des groupes de Lie nilpotents. III, Canad. J. Math. 10 (1958), 321-348. MR 20 \#1929.

4. , Représentations irréductibles des algèbres de Lie nilpotentes, An. Acad. Brasil. Ci. 35 (1963), 491-519. MR 32 \# 165.

5. __ Algèbres enveloppantes, Gauthier-Villars, Paris, 1974.

6. G. B. Folland, Subelliptic estimates and function spaces on nilpotent Lie groups, Ark. Mat. 13 (1975), 161-207.

7. G. B. Folland and E. M. Stein, Estimates for the $\overline{\partial_{b}}$ complex and analysis on the Heisenberg group, Comm. Pure Appl. Math. 27 (1974), 429-522. MR 51 \#3719.

8. V. V. Grušin, Pseudodifferential operators on $R^{n}$ with bounded symbols, Funkcional. Anal. i Priložen 4 (1970), no. 3, 37-50 = Functional Anal. Appl. 4 (1970), no. 3, 202-212. MR 42 \#5106.

9. 456-473 = Math. USSR-Sb. 12 (1970), 458-476. MR 43 \#5158.

10. $\quad$ A certain class of elliptic pseudodifferential operators that are degenerate on a submanifold, Mat. Sb. (N.S.) 84 (126) (1971), no. 2, 163-195 = Math. USSR-Sb. 13 (1971), 155-185. MR 44 \#860.

11. V. W. Guillemin, Singular symbols (to appear).

12. S. Helgason, Differential geometry and symmetric spaces, Academic Press, New York, 1962. MR 26 \#2986.

13. L. Hörmander, Linear partial differential operators, Grundlehren math. Wiss., Bd. 116, Springer-Verlag, Berlin; Academic Press, New York, 1963. MR 28 \#4221.

14. Hypoelliptic second order differential equations, Acta Math. 119 (1967), 147-171. MR 36 \#5526.

15. A. A. Kirillov, Unitary representations of nilpotent Lie groups, Uspehi Mat. Nauk 17 (1962), no. 4 (106), 57-110 = Russian Math. Surveys 17 (1962), no. 4, 53-104. MR 25 \#5396.

16. __ Plancherel measure for nilpotent Lie groups, Funkcional. Anal. i Priložen. 1 (1967), no. 4, 84-85 = Functional Anal. Appl. 1 (1967), no. 4, 330-331. MR 31 \#347.

17. A. Messiah, Quantum mechanics, Vol. I, Wiley, New York, 1968.

18. E. Nelson, and W. F. Stinespring, Representation of elliptic operators in an enveloping algebra, Amer. J. Math. 81 (1959), 547-560. MR 22 \#907.

19. Y. Nouazé and P. Gabriel, Idéaux premiers de l'algèbre enveloppante d'une algèbre de Lie nilpotente, J. Algebra 6 (1967), 77-99. MR 34 \#5889.

20. M. Rais, Solutions élémentaires des opérateurs différentiels bi-invariants sur un groupe de Lie nilpotent, C.R. Acad. Sci. Paris Sér. A-B 273 (1971), A495-A498. MR 44 \#6908.

21. C. Rockland, Hypoellipticity and eigenvalue asymptotics, Lecture Notes in Math., vol. 464, Springer-Verlag, Berlin and New York, 1975. 
22. L. P. Rothschild and E. M. Stein, Hypoelliptic differential operators and nilpotent groups, Acta Math. 137 (1976), 247-320.

23. E. M. Stein, Some problems in harmonic analysis suggested by symmetric spaces and semi-simple groups, Actes Congrès Internat. Mathématiciens (Nice, 1970), Vol. I, GauthierVillars, Paris, 1971, pp. 173-189.

24. F. Trèves, Topological vector spaces, distributions and kernels, Academic Press, New York, 1967. MR 37 \# 726.

\section{SUPPLEMENTARY REFERENCES}

25. R. Beals, Séminaire Goulaouic-Schwartz, 1977.

26. __ Computes rendues du colloque de St.-Jean de Monts, June 1977.

27. P. Cartier, Quantum mechanical commutation relations and theta functions, Proc. Sympos. Pure Math., vol. 9, Amer. Math. Soc., Providence, R.I., 1965, pp. 361-387.

28. A. Dynin, Pseudodifferential operators on Heisenberg groups (Seminar given at C.I.M.E. Bressanone, Italy, June 1977).

29. B. Helffer, Hypoellipticité pour des opérateurs differentiels sur des groupes de Lie nilpotents, Centre Math. Ecole Polytechnique, Palaiseau, France (preprint).

Department of Mathematics, Brandeis University, Waltham, MassachusetTs 02154 